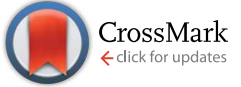

Cite this: RSC AdV., 2017, 7, 14114

Received 6th December 2016 Accepted 22nd February 2017

DOI: 10.1039/c6ra27830b

rsc.li/rsc-advances

\section{An overview of chemical constituents from Alpinia species in the last six decades $\uparrow$}

\author{
Xiao-Ni Ma, ${ }^{\text {ab }}$ Chun-Lan Xie, ${ }^{\text {ab }}$ Zi Miao, ${ }^{a}$ Quan Yang $^{\mathrm{b}}$ and Xian-Wen Yang*a \\ Alpinia species is one of the most important genera of the Zingiberaceae family. In Asia, they have been \\ widely used as food and traditional medicines for centuries. This review focuses on their chemical \\ constituents and their relevant biological activities with 252 references covering from 1955 to 2015. In \\ total, 544 compounds were isolated from 35 Alpinia species. The major ones are terpenoids (207) and \\ diarylheptanoids (143). The crude extracts and identified compounds exhibited a broad spectrum of \\ bioactivities including antiemetic, antiulcer, antibacterial, anti-inflammatory, anti-amnesic, anticancer, etc.
}

\section{Introduction}

The genus Alpinia is an important member of the Zingiberaceae family. It includes ca. 230 species. ${ }^{1}$ Most of them are distributed in tropical and subtropical Asia, including India, Malaysia, China, and Japan. A few are found in Australia and the Pacific Islands. ${ }^{1-3}$ Plants of this genus have been extensively used for different purposes for centuries. For example, A. vittata, A. purpurata (Vieill.) K. Schum., A. calcarata Rosc., and A. zerumbet are cultivated as ornamental plants; ${ }^{3,4}$ A. blepharocalyx $\mathrm{K}$. Schum. is a natural dye; A. galanga (L.) Willd is an important ingredient for curries and has been broadly utilized as a flavoring in the preparation of meats and soups in Southeast Asia ${ }^{6-8}$ and in the preparation of beverages in Europe; ${ }^{9}$ and $A$. officinarum Hance, listed as medicinal and edible food by the Chinese Ministry of Health, are used in medicinal diets, ${ }^{10-15}$ wines, ${ }^{16}$ sauces, and flavorings. ${ }^{17-19}$ Moreover, A. galanga (L.) Willd is also applied to preserve food and fruits. ${ }^{\mathbf{8} 20}$ Most important of all, Alpinia plants are also broadly used as traditional medicines in India, China, and Japan to treat many diseases such as indigestion, gastralgia, vomiting, enterozoa etc. $^{21-23}$ Thus, a growing investigation on the chemical constituents and bioactivities of this genus has been carried out since 1955. ${ }^{24}$ Consequently, Alpinia species were proved to have various biological activities including antiulcer, ${ }^{25}$ antiemetic, ${ }^{26-28}$ antibacterial, ${ }^{29-31}$ antitumor, ${ }^{32-34}$ hypoglycemic, ${ }^{35}$ cardioprotection, ${ }^{36}$ antifungi, ${ }^{37}$ neuroprotection, ${ }^{38,39}$ and antianxiety activities. ${ }^{40}$

\footnotetext{
${ }^{a}$ State Key Laboratory Breeding Base of Marine Genetic Resources, Key Laboratory of Marine Genetic Resources, Fujian Key Laboratory of Marine Genetic Resources, Third Institute of Oceanography, State Oceanic Administration, 184 Daxue Road, Xiamen 361005, PR China. E-mail: yangxianwen@tio,org,cn

${ }^{b}$ Department of Traditional Chinese Medicine, Guangdong Pharmaceutical University, Guangzhou 510006, China

$\dagger$ Electronic supplementary information (ESI) available: The name, source, plant part, and reference for each compound. A comparison of Alpinia species names from the references and the accepted name in The Plant List. See DOI: 10.1039/c6ra27830b
}

Up to 2015, this genus contributed about 252 papers. However, only seven review articles were published, five of which were on chemical constituents and biological activities of single plant. And the rest two were on two major components of Alpinia species. The first review came out in 2010 regarding distributions, physiological activities and ${ }^{13} \mathrm{C}$ NMR spectroscopic data of 307 naturally occurring diarylheptanoids, which were mainly isolated from Alpinia species. ${ }^{41}$ In 2011, the pharmacological and phytochemical studies of A. galanga (L.) Willd were summarized with 30 references. Although it was claimed to concern new phytoconstituents that have appeared in recent years for A. galangal, it actually collected all reported compounds including volatile oil. ${ }^{\mathbf{4 2}}$ In 2012, structural characterization and biological effects of constituents from the seeds of A. katsumadai was described. Sixty compounds were reported together with their structures and bioactivities with 18 references. ${ }^{43}$ In 2013 , chemical constituents in fruits of $A$. oxyphylla and their pharmacological activities were summarized. Eighty-five compounds were obtained from this species between 2001 and 2012, with the major component of sesquiterpenes (61.2\%). It possessed a variety of pharmacological activities, including neuroprotection, learning and memoryimproving function, anticancer, anti-aging, anti-inflammation, and anti-anaphylaxis. ${ }^{44}$ In 2015 , a comprehensive review on the ethnomedical uses, chemical constituents, and the pharmacological profile of $A$. calcarata Roscoe was published with particular attention given to the pharmacological effects of the essential oil. ${ }^{45}$ In the same year, the phytochemistry of A. purpurata with pharmacological properties of antioxidant, antibacterial, larvicidal, cytotoxic, and vasodilator activities were reported together with another ornamental ginger, Hedychium coronarium. As a matter of fact, little research was performed on A. purpurata. ${ }^{46}$ In addition, the isolation, synthesis, and characterization of dihydro-5,6-dehydrokavain, the major constituent of A. zerumbet were also reviewed. ${ }^{47}$ However, so far there has been no comprehensive review for chemical constituents of this species. Herein, we describe all isolated compounds and their 
relevant bioactivities of Alpinia species reported in the last six decades from 1955 to 2015.

\section{Terpenoids}

\subsection{Monoterpenoids}

A total number of 17 monoterpenoids were obtained from Alpinia species (Fig. 1). Rubraine (1), isorubraine (2), and sumadain C (3) were three new monoterpene-chalcone conjugates obtained from $A$. katsumadai.$^{48}$ They were tested for cytotoxic activities against three tumor cell lines of HepG2, MCF-7, and MAD-MB-435. Sumadain C (3) exhibited very weak effect with $\mathrm{IC}_{50}$ value of around $40.0 \mu \mathrm{M} \cdot{ }^{48} \mathrm{~A}$. katsumadai Hayata yielded a new monoterpene-kavalactone conjugate, katsumadain (4) and a new (E)-1-(1-terpinen-4-olyl)-3methoxystilbene (5). ${ }^{49}$ While A. densibracteata $\mathrm{T}$. L. Wu and Senjen yielded two diastereoisomers of cinnamate esters, $2 \alpha$-cinnamoyl cineole (6) and $2 \beta$-cinnamoyl cineole (7). ${ }^{50}$ From rhizomes of $A$. tonkinensis Gagnep., $2 \alpha$ - $(p$-hydroxycinnamoyl) cineole (8) was isolated. ${ }^{50,51}$ Two endoperoxides, $(1 S, 4 R, 6 R)$-1,4-epidioxy-p-menth-2-ene (9) and $(1 R, 4 S, 6 R)$-1,4-epidioxy-p-menth-2-ene (10), were isolated from aerial parts of $A$. densibracteata T. L. Wu and Senjen. ${ }^{50}$ Whilst $(3 R, 4 R, 6 S)$-3,6-dihydroxy-1-menthene (11) and 1-terpinen-4-ol (12) were obtained from A. sichuanensis Z. Y. Zhu (a synonym of $A$. jianganfeng $\mathrm{T}$. L. $\mathrm{Wu}$ ) and $A$. katsumadai Hayata, respectively. ${ }^{49,52}$ Fruit of $A$. oxyphylla Miq. was the source of $(1 R, 2 R)-p$-menth-3-ene1,2-diol (13). ${ }^{53}$ And aerial parts of A. densibracteata T. L. Wu and Senjen yielded 3,4-dihydroxy-p-menth-1-ene (14). ${ }^{50}$ Compounds 1517 were three hydroxyl-1,8-cineole glucopyranosides, which were mainly isolated from rhizomes of A. galanga (L.) Willd. ${ }^{54,55}$

\subsection{Sesquiterpenoids}

To date, 132 sesquiterpenoids were reported from Alpinia species (Fig. 2). They were divided into acyclic sesquiterpenoids (18 and 19), eremophilanes (20-40), eudesmanes (41-84), cadinanes (85100), guaianes (101-117), caryophyllanes (118-120), bisabolanes (121-137), humulanes (138-140), drimane (141), elemane (142), carabrane (143), oplopanane (144), and others (145-149).

Seeds of A. katsumadai Hayata produced an acyclic sesquiterpenoid, trans,trans-farnesol (18), which exerted weak neuraminidase inhibitory activity in vitro $\left(\mathrm{IC}_{50}=81.4 \mu \mathrm{M}\right){ }^{56}$ Nerolidol (19), another acyclic sesquiterpene, was obtained from rethizoms of $A$. japonica. ${ }^{57}$

Investigations on fruits of A. oxyphylla Miq. afforded 16 eremophilanes (20-35). Epinootkatol (29) and nootkatone (30) displayed insecticidal activities against larvae and adults of Drosophila melanogaster with $\mathrm{IC}_{50}$ values of $11.5 \mu \mathrm{M}$ and $96 \mu \mathrm{g}$ per adult, respectively. ${ }^{58}$ While $9 \beta$-hydroxynootkatone (31), (11S)12-chloronootkaton-11-ol (32), and (11R)-12-chloronootkaton11-ol (33) displayed anti-acetylcholinesterase (AChE) activities by TLC-bioautographic assays..$^{59,60} 12$-Nornootkaton-6-en-11-one (35) was a novel nor-eremophilane. It showed potent antiAChE bioactivity at $10 \mathrm{nM}$ using the same TLC-bioautographic assay. ${ }^{59}$ The rest of five eremophilanes (36-40) were isolated from three different species. Eremophilen-10 $\beta$-ol (36) and eremophilen-11-ol (37) were obtained from A. intermedia Gagnep. and A. japonica (Thunb.) Miq., respectively, ${ }^{61,62}$ whilst nootkatene (38), valencene (39), and dehydro-nootkatone (40) were all identified from A. oxyphylla Miq. ${ }^{59,63-65}$

Among 44 eudesmane sesquiterpenoids, oxyphyllones A and B (41 and 42) were isolated from A. oxyphylla. They were the first two examples of 4,5-secoeudesmanes in the Zingiberaceae family ${ }^{66}$ Oxyphyllone A displayed moderate anti-AChE activity. ${ }^{59}$ Also obtained from A. oxyphylla Miq. were compounds 43-63..$^{67,68}$ A. intermedia Gagnep. was the source of intermedeol (64) and $\beta$ selinene (65). ${ }^{61}$ Investigations of A. japonica (Thunb.) Miq. led to the identification of 66-75..$^{21,57,69,70}$ Two novel trinoreudesmanes, oxyphyllanenes A (76) and B (77) were obtained from A. oxyphylla, together with four known ones (78-81). ${ }^{71,72}$ Investigation on $A$. oxyphylla Miq. provided three nor-eudesmane sesquiterpenoids, oxyphyllanene $\mathrm{C}$ (82), (5R,7S,10S)-5-hydroxy-13-noreudesma-3en-2,11-dione (83), and 4-methoxy-oxyphyllenone A (84)..$^{67,71,73}$

A new 1,10-seco-15-norcadinane sesquiterpene nominated oxyphenol A (85) was isolated from A. oxyphylla. ${ }^{65}$ Fruits of A. oxyphylla Miq. also provided one tricyclic sesquiterpene, mustakone (86), nine nor-cadinanes, 87-94 and $2 \beta$-hydroxy- $\delta$-cadinol $(95){ }^{53,59,68,74} A$. oxymitra K. Schum. was the source of $(-)-(1 R, 4 S)-8$-hydroxy-13calamenenoic acid (96). ${ }^{75}$ Alpiniaterpene A (97) was provided by A. officinarum Hance, ${ }^{76}$ while 4(15)-cadinene-6,10-diol (98) by $A$. tonkinensis Gagnep. ${ }^{51}$ Two new compounds (99 and 100) were isolated from fruits of $A$. oxyphylla Miq. And 100 exhibited moderate hypoglycemic activity with inhibitory rate of $11.5 \%$, compared to $41.9 \%$ of the positive control acarbose (41.9\%) at $90 \mu \mathrm{M} .^{77}$

Rhizomes of A. japonica (Thunb.) Miq. produced alpinenone (101), an inhibitor of AChE. ${ }^{59,60}$ Hanamyol (102), containing a cyclic ether linkage, was also isolated from A. japonica (Thunb.) Miq. ${ }^{78}$ Rhizomes of $A$. intermedia Gagnep. provided hanalpinol peroxide (103), isohanalpinol (104), and aokumanol (105). ${ }^{61}$ While A. intermedia Gagnep. and A. japonica (Thunb.) Miq. produced hanalpinol (106), hanalpinone (107), and isohanalpinone (108). ${ }^{61,79}$ From A. japonica (Thunb.) Miq. and A. intermedia Gagnep., furopelargones A (109) and B (110) were obtained. ${ }^{\mathbf{6 1 , 7 8 , 8 0}}$ Later on, $\mathbf{1 1 0}$ was also found from A. formossana ${ }^{81}$ Compounds 111-114 were four secoguaiane-type sesquiterpenes with an $\alpha, \beta$-unsaturated butenolide. A. intermedia Gagnep. produced epialpinolide (111), whilst A. japonica (Thunb.) Miq. yielded alpinolide peroxide (112), 6-hydroxy-alpinolide (113), and alpinolide (114). ${ }^{61,78,79} \mathrm{~A}$ 1,10-secoguaiane sesquiterpene, (+)-mandassidion (115), and two 1,10-seco-15-norguaiane sesquiterpenes, mandassions A (116) and B (117) were obtained from fruits of A. oxyphylla Miq. ${ }^{65}$

Caryophyllene oxide (118), caryophyllenol-I (119), and caryophyllenol-II (120) were caryophyllanes from A. galanal. In addition, caryophyllene oxide was also distributed in rhizomes of A. conchigera Griff. $^{24,82}$

Investigation of the aerial parts of $A$. densibracteata T. L. Wu and Senjen led to the isolation of two bisabolane endoperoxides (121 and 122), three bisabolane hydroperoxides (123-125), and one 3,4-dihydroxy-bisabola-1,10-diene (126). ${ }^{50}$ Compounds 127137 were reported from rhizomes of A. japonica (Thunb.) Miq. ${ }^{83}$

A. oxyphylla Miq. was the source of $3(12), 7(13), 9(E)-$ humulatriene-2,6-diol (138). ${ }^{84}$ While A. formossana and A. japonica produced humulene epoxideII (139). ${ }^{57,81}(9 E)$-Humulene-2,3;6,7diepoxide (140) was reported from the fruits of $A$. oxyphylla Miq. However, its relative configuration remained undetermined. It 
<smiles>CC1(C)Oc2c(C(=O)/C=C/c3ccccc3)c(O)cc3c2C2CCC[C@](C)(CC21)O3</smiles>

Rubraine

1<smiles>COc1cc(/C=C/c2ccccc2)cc(OC2(C(C)C)CC=C(C)CC2)c1</smiles>

(E)-1-(1-Terpinen-4-olyl)-3-methoxystilbene

5

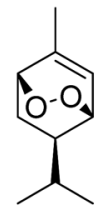

(IS,4R,6R)-1,4-Epidioxyp-menth-2-ene 9<smiles>CC(C)C1C=CC(C)(O)[C@@H](O)C1</smiles>

14<smiles></smiles><smiles>CC12CCC(C)(C)C(C)(CC1)OC2OC(=O)/C=C/c1ccccc1</smiles>

$2 a$-Cinnamoyl cineole

6<smiles>CC1=C[C@H](O)[C@H](C(C)C)C[C@H]1O</smiles>
-1-menthene

11
$(3 R, 4 R, 6 S)-3,6$-Dihydroxy<smiles>CC[C@H]1C=C[C@@]2(C)Oc3c(C(=O)/C=C/c4ccccc4)c(O)cc(O)c3C2C1</smiles><smiles>CC1=C[C@H](c2c(O)cc(/C=C/c3ccccc3)oc2=O)C(C(C)C)CC1</smiles>

Katsumadain

4<smiles>CC1(C)CCC2(C)CCC1OC2OC(=O)/C=C/c1ccccc1</smiles>

$2 \beta$-Cinnamoyl cineole

7<smiles>CC1=CCC(O)(C(C)C)CC1</smiles>

1-Terprnen-4-ol

12

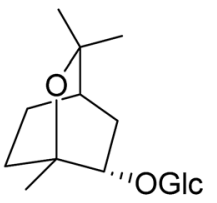

(1S,2S,4R)-trans-2-Hydroxy-1,8cineole $\beta$-D-glucopyranoside

16<smiles>CC1(C)CC2CCC1(C)C(OCl)[C@H]2Cl</smiles>

(1R,3S,4S)-trans-3-Hydroxy-1,8cineole $\beta$-D-glucopyranoside

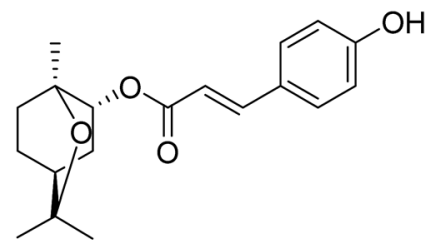

$2 \alpha-(p-H y d r o x y c i n n a m o y l)$ cineole

8<smiles>CC(C)C1=C[C@@H](O)[C@](C)(O)CC1</smiles>

$(1 R, 2 R)-p-M e n t h-$ 3-ene-1,2-diol

13 17

Fig. 1 Monoterpenoids from Alpinia species.

exhibited moderate anti-AChE activity in bioautographic assay at $10 \mathrm{nM} .^{\mathbf{5 9 , 8 4}}$ Interestingly, the structure and molecular formula for 140 (CAS Registry Number: 21956-93-4) provided by Scifinder were not correct. It should be $\mathrm{C}_{15} \mathrm{H}_{24} \mathrm{O}_{2}$ instead of $\mathrm{C}_{14} \mathrm{H}_{21} \mathrm{O}_{2}$.

Rhizomes of $A$. calcarata Rosc. affored a drimane-type sesquiterpene $\left(\gamma\right.$-bicyclohomofarnesal, 141) ${ }^{85}$ and an elemane one (shyobunone, 142). ${ }^{83}$ Pubescone (143) was isolated from $A$. oxyphylla Miq. and showed weak anti-AChE activity at the concentration of $100 \mu \mathrm{M} .^{59}(-)$-Oplopanone (144) and oxyphyllone F (145) were obtained from fruits of $A$. oxyphylla Miq. ${ }^{84}(Z)-4-(2,6-$ Dimethylhepta-1,5-dien-1-yl)-1-methyl-cyclobut-1-ene (146) was a novel nor-sesquiterpene incorporating cyclobutene ring from $A$. oxyphylla Miq. ${ }^{74}$ Seeds of A. galanga (L.) Willd produced caryolane1,9 $\beta$-diol (147), which suppressed the proliferation of four cancer cell lines of HeLa, A549, HepG2, and SMMC-7721 with $\mathrm{IC}_{50}$ values ranged from 252 to $378 \mu \mathrm{M}^{86}$ A. japonica (Thunb.) Miq. yielded alpiniol (148). ${ }^{87}$ Compound 2-ethyl-6-isopropyl-7-hydroxymethyl naphthalene (149) was a noval naphthalene from $A$. oxyphylla. ${ }^{77}$ It showed bioactive activity with the inhibitory rates of $10.3 \%$, compare to $41.9 \%$ of the positive control acarbose at $0.9 \mathrm{mM} .{ }^{77}$

Noteworthily, compounds 22-31, 34, 48-56, 58-63, 79-82, 87$\mathbf{8 9}, \mathbf{1 1 7}$, and 129-136 exerted NO production inhibitory activities at different levels. ${ }^{58-60,65,67,71,73,83,88-90}$ While (10R)-13-noreudesma4,6-dien-3,11-dione (46), (5S,8R,10R)-2-oxoeudesma-3,7(11)-dien12,8-olide (47), (5R,7S,10S)-5-hydroxy-13-noreudesma-3-en-2,11dione (83), and (4S)-10-nor-calamenen-10-one (90) showed potent auxo-action of NO production at $10 \mu \mathrm{M}$ induced by lipopolysaccharide (LPS) in microglia. ${ }^{71}$

\subsection{Diterpenoids}

Labdane diterpenes is undoubtedly predominant in Zingiberaceae family, notably in Alpinia genus. Almost all diterpenes are 
<smiles>CC(C)=CCCC(C)=CCCC(C)=CCO</smiles>

trans,trans-Farnesol

18<smiles>C=C(C)[C@@H]1C[C@@H](O)C2=C[C@H](O)C[C@H](C)[C@]2(C)C1</smiles>

9-Hydroxy epinootkatol 23<smiles>[R]C1C=C2CC[C@@H](C(=C)C)C[C@]2(C)[C@H](C)C1</smiles>

$28 \mathrm{R}=\boldsymbol{\beta}-\mathrm{OH}$ Nootkatol $29 \mathrm{R}=\alpha-\mathrm{OH}$ Epinootkatol<smiles>CC(=O)[C@@H]1CCC2=CC(=O)C[C@H](C)[C@]2(C)C1</smiles>

$\left(4 R^{*}, 5 S^{\star}, 7 R^{\star}\right)-7$-Acetyl-4,5dimethyl-4,5,6,7,8,9-hexahydronaphthalen-2(3H)-one<smiles>C=C(C)[C@@H]1CCC2=CCC[C@H](C)[C@@]2(C)C1</smiles>

Valencene

39<smiles>C=C(C)[C@]1(O)CC[C@@]2(C)CCC(=O)C3(C)OC32C1</smiles>

Oxyphyllol E $\quad(4 a$

44<smiles>CC(O)(CO)C1=CCCC1</smiles>

$4811 \beta$-Hydroxyl-3-oxo- 4(5),6(7)diene-eude-sman-12-ol

49 11a-Hydroxyl-3-oxo- 4(5),6(7)diene-eudes-man-12-ol<smiles>C=C[C@](C)(O)CC/C=C\CCC=C(C)C</smiles>

Nerolidol

19<smiles>C=C(C)C1CC(=O)C2=CC(=O)C[C@H](C)[C@]2(C)C1</smiles>

Eremophila-1(10),11(12)dien-2,9-dione

24<smiles>C=C(C)[C@@H]1CCC2=CC(=O)C[C@H](C)[C@]2(C)C1</smiles>

Nootkatone

30<smiles>CC(=O)C1=C[C@]2(C)C(=CC(=O)C[C@H]2C)CC1</smiles>

12-Nornootkaton-6-en-11-one

35<smiles>C=C(C)C1C=CC2=CC(=O)C[C@H](C)[C@]2(C)C1</smiles>

Dehydro-nootkatone

40<smiles>C=C(C)[C@H]1CC[C@]2(C)CCC(=O)C(C)=C2[C@H]1O</smiles>

8-Hydroxy-1,4a-dime

exahydronaphthalen-2(3H)-one

45<smiles>C=C(C)[C@]1(O)CC[C@@]2(C)CC(=O)C=C(C)[C@H]2C1</smiles>

Teucrenone

51<smiles>C[C@H]1CC(=O)C=C2CC[C@@H](C(C)(C)O)C[C@]21C</smiles>

11-Hydroxyvalenc-1(10)-en-2-one<smiles>C=C(C)C1C[C@H](O)C2=CC(=O)C[C@H](C)[C@]2(C)C1</smiles>

$9 \beta$-Hydroxynootkatone 31

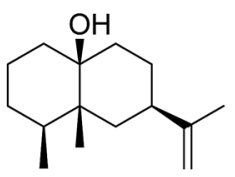

Eremophilen- $10 \beta$-ol

36<smiles>CC(=O)CCC[C@H]1CCC(C(C)=O)=CC1=O</smiles>

41<smiles>CC(=O)C1=CC2=C(C)C(=O)CC[C@]2(C)CC1</smiles>

(10R)-13-Noreudesma-

4,6-dien-3,11-dione

46<smiles>C=C(C)[C@H]1CC[C@]2(C)CCC(=O)C(C)=C2[C@H]1O</smiles>

(4a S,7S,8R)-8-Hydroxy-1,4a-dimeth 7-(prop-1-en-2-yl)-4,4 $\alpha, 5,6,7,8$ hexahydronaphthalen-2(3H)-one

52<smiles>C[C@H]1CC(=O)C=C2CC[C@](CO)([C@@](C)(O)CO)C[C@]21C</smiles>

(11S)-Nootkatone-11,12-diol

21<smiles>C=C(C)[C@@H]1C[C@@H](O)C2=CC(=O)C[C@H](C)[C@]2(C)C1</smiles>

Oxyphyllol B

26<smiles>C[C@H]1CC(=O)C=C2CC[C@@H]([C@@](C)(O)CO)C[C@]21C</smiles>

(11R)-Nootkatone-11,12-diol

22<smiles>C=C(CO)[C@@H]1CCC2=CC(=O)C[C@H](C)[C@]2(C)C1</smiles>

13-Hydroxynootkatone

27<smiles>C[C@H]1CC(=O)C=C2CC[C@H]([C@@H](O)CCl)C[C@]21C</smiles>

(11R)-12-Chloronootkaton-11-ol

33<smiles>C=C(C)[C@@H]1CC=C2C=CC[C@H](C)[C@@]2(C)C1</smiles>

Nootkatene

38<smiles>CC(=O)CCC[C@]1(C)CCC(C2(C)CO2)=CC1=O</smiles>

42<smiles>CC1=CC(=O)C[C@]2(C)CC3OC(=O)C(C)=C3C[C@H]12</smiles>

$(5 S, 8 R, 10 R)-2-$ Oxoeudesma-

3,7(11)-dien-12,8-olide

47<smiles>CC1=C2[C@@H]3OC[C@](C)(O)[C@@]3(O)CC[C@]2(C)CCC1</smiles><smiles>CC1=C2C[C@H](C(C)(C)CO)CC[C@]2(C)CCC1=O</smiles>

$7 a(H), 10 \beta$-Eudesm-

4-en-3-one-11,12-diol

50<smiles>C=C(C)[C@@H]1CC[C@H]2C(=C(C)C(=O)C[C@H]2O)C1</smiles><smiles>C=C(C)[C@]1(O)CC[C@]2(C)CCC(=O)C(C)=C2C1</smiles>

(4a S,7S)-7-Hydroxy-1,4a-dimethyl7-(prop-1-en-2-yl)-4,4 $\alpha, 5,6,7,8$ hexahydronaphthalen-2(3H)-one

53

Fig. 2 Sesquiterpenoids from Alpinia species.

labdanes (150-205). Only one grayanane diterpene was found (206) (Fig. 3).

(E)-Labda-8(17),12-diene-15,16-dial (150) is widely distributed in Alpinia. It exhibited a number of bioactivities, such as antibacterial, ${ }^{91} \alpha$-glucosidase inhibition, ${ }^{92}$ NO production inhibition, ${ }^{88}$ antifungal, ${ }^{93}$ antiglycation, ${ }^{94}$ HIV-1 integrase, and neuraminidase inhibitory activities. ${ }^{95}$ A. katsumadai Hayata, A. galanga (L.) Willd, and $A$. nigra yielded (E)-8 $\beta, 17$-epoxylabd-12- 
<smiles>C=C(C)C1CC[C@]2(C)[C@@H](O)CC[C@@H](O)[C@H]2C1</smiles>

$( \pm) 1 \beta, 4 \beta$-Dihydroxyeudesman-11-ene<smiles>C=C1[C@@H](O)CC[C@@]2([13CH3])CC[C@@](O)(C(=C)C)C[C@H]12</smiles>

Oxyphyllanene E 60<smiles>C=C1CCC[C@]2(C)CC[C@@H](C(=C)C)C[C@H]12</smiles>
$\beta$-Selinene

65

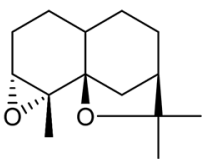

$3 a, 4 a$-Oxidoagarofuran

70

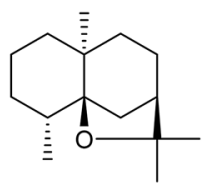

Dihydroagarofuran

75<smiles>[R]C1CC[C@]2(C)CCC(=O)C=C2C1([R6])[R6]</smiles>

$$
\begin{array}{lll}
R_{1} & R_{2} & R_{3} \\
\hline 8 & O H & O H
\end{array}
$$

$80 \mathrm{OH} \mathrm{OH} \mathrm{Me} \mathrm{Oxyphyllenone} A$

$81 \mathrm{OH} \mathrm{Me} \mathrm{OH}$ Oxyphyllenone B

$84 \mathrm{OH} \mathrm{OMe}$ Me 4-Methoxy-oxyphyllenone A<smiles>CC(C)[C@H]1CCC(=O)C2=C1[C@@H](O)[C@](C)(O)CC2</smiles>

8765 Oxyphyllenodiol A $886 R$ Oxyphyllenodiol B<smiles>C=C(C)C1CCC2(C)CC[C@H](O)C(=C)[C@H]2C1</smiles>

56<smiles>CC1=C2C=C([C@@](C)(O)CO)CC[C@]2(C)CCC1=O</smiles>

Oxyphyllanene $\mathrm{F}$

61<smiles>CC1=C2C[C@H](C(C)(C)O)CC[C@]2(C)CCC1</smiles>

10-epi-y -Eudesmol

66

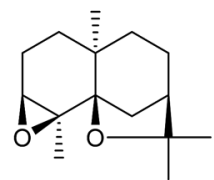

71

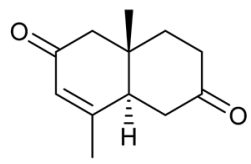

Oxyphyllanene A

76<smiles>CC(=O)[C@@]12CC[C@@]3(C)CCC(=O)C(C)=C3[C@@H]1O2</smiles>

Oxyphyllanene C

82<smiles>[R]c1c(C)ccc2c1[C@H](C(C)C)CCC2=O</smiles>

$89 \mathrm{R}=\mathrm{OH}$ Oxyphyllone $\mathrm{G}$

$90 \mathrm{R}=\mathrm{H}$ (4S)-10-Nor-calamenen-10-one<smiles>CC1=C2C=C([C@](C)(O)CO)CC[C@]2(C)CCC1=O</smiles>

Oxyphyllanene $G$

57

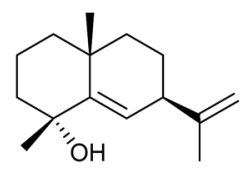

Oxyphyllol A

62

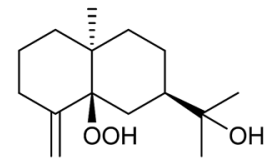

10-epi-5 $\beta$-Hydroperoxy

$-\beta$-eudesmol

67

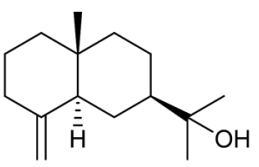

$\beta$-Eudesmol

72<smiles>CC1=CC(=O)C[C@]2(C)CCC(=O)C=C12</smiles>

Oxyphyllanene B

77

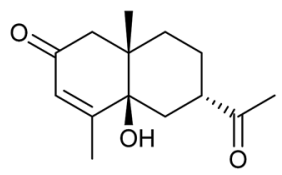

(5R,7S,10S)-5-Hydroxy-13noreudesma-3-en-2,11-dione

83<smiles>CC(C)[C@H]1CCC(=O)C2=C1[C@H]1O[C@@]1(C)CC2</smiles>

Oxyphyllone C

91<smiles>C=C(C)[C@]1(O)CC[C@@]2(C)CC(=O)C=C(C)[C@H]2C1</smiles>

7-epi-Teucrenone

58

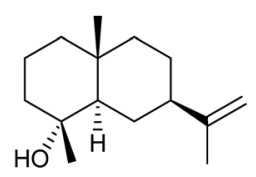

Selin-11-en-4 $\alpha$-ol

63

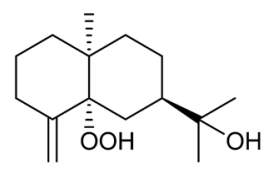

10-epi-5 $\alpha$-Hydroperoxy

$-\beta$-eudesmol

68

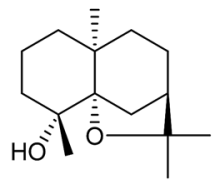

4-Hydroxy-dihydroagarofuran

73<smiles>C[C@]1(O)CCC[C@]2(C)CCC(=O)C=C12</smiles>

$\left(4 S^{*}, 5 E, 10 R^{*}\right)-7-0 x o-t r i-n o r-$

eudesm-5-en-4 $\beta$-ol

78<smiles>COC(=O)CC[C@@H](c1cc(C)ccc1O)C(C)C</smiles>

Oxyphenol A

85<smiles>C=C(C)C1CC[C@]2(C)CCC[C@](C)(O)[C@H]2C1</smiles>

Intermedeol

64<smiles>C[C@H]1CCC[C@]2(C)CCC(C(C)(C)O)C[C@]12O</smiles>

4,10-epi-5 $\beta$-Hydroxy-

dihydroeudesmol

69

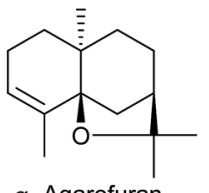

$a$-Agarofuran

74<smiles>C[C@]1(O)CCC[C@]2(C)CCC(=O)C=C12</smiles>

Teuhetenone A

79

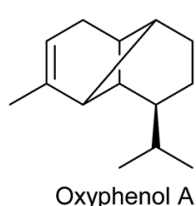

86

Fig. 2 Sesquiterpenoids from Alpinia species (continued).

ene-15,16-dial (151). It exhibited extensive antibacterial activities, especially against Candida guilliermondii and Candida tropicalis. ${ }^{49,91,93,96}$ Moreover, 151 also showed $\alpha$-glucosidase inhibitory activity with $\mathrm{IC}_{50}$ value between $5 \mu \mathrm{M}$ and $10 \mu \mathrm{M}^{70}$ The $\alpha$-glucosidase inhibitory activity of $\mathbf{1 5 1}$ was even much higher than the positive control, acarbose $\left(\mathrm{IC}_{50}=400 \mu \mathrm{M}\right)$, 
<smiles>CC(C)c1ccc(O)c2c1[C@H](O)[C@](C)(O)CC2</smiles>

Oxyphyllenotriol A

94<smiles>CC1=C[C@H]2C(C(C)C)CC[C@@H](O)[C@H]2CC1O</smiles>

$2 \beta$-Hydroxy- $\delta$-cadinol

95<smiles>CC(C)C1CC[C@@H](C)c2c(O)cc(C(=O)O)cc21</smiles>

(-)-(1R,4S)-8-Hydroxy-

13-calamenenoic acid<smiles>C=C1CC[C@H]([C@@H](C)C(=O)O)[C@@H]2C=C(C(=O)OC)CC[C@@H]12</smiles>

Alpiniaterpene $\mathrm{A}$

97<smiles>C=C1CC[C@H]2[C@@H](C)CCC(C(C)C)[C@@]2(O)C1</smiles>

4(15)-Cadinene-6,10-diol

98<smiles>CC1CCC(O)(C(C)C)C2=CC(=O)CC[C@@H]21</smiles>

$99 \quad \alpha$ 2-methyl-6-isopropyl-7hydroxymethyl naphthalene<smiles>CC(C)C1=CC(=O)[C@H]2O[C@H]3[C@@H](C)CC[C@H]3[C@H]2[C@H]1O</smiles>

Alpinenone 101

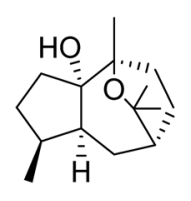

Hanamyol

102

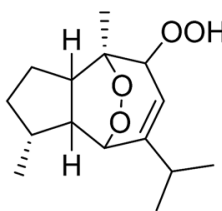

Hanalpinol peroxide

103

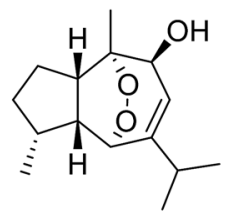

Isohanalpinol

104

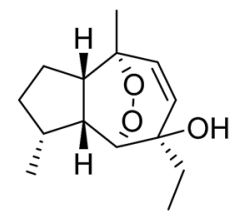

Aokumanol

105 $100 \beta$ epi-oxyphyllenone<smiles>CC(C)C1=C[C@H](O)[C@H]2O[C@H]1[C@H]1CC[C@H](C)[C@H]1[C@H]2C</smiles>

Hanalpinol

106

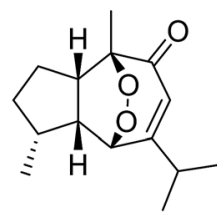

Hanalpinone

107

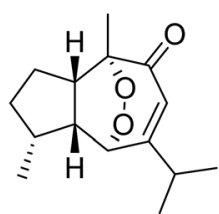

Isohanalpinone

108

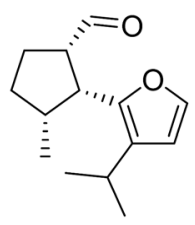

Furopelargone B 109<smiles>CC(C)c1ccoc1[C@H]1[C@H](C)CC[C@H]1C=O</smiles>

Furopelargone $\mathrm{A}$ 110

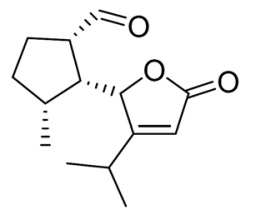

Epialpinolide

111<smiles>CC(C)C1=CC(=O)O[C@]12OOC(C)(O)[C@H]1CC[C@@H](C)[C@H]12</smiles>

Alpinolide peroxide 112<smiles></smiles>

Caryophyllene oxide 118<smiles>CC(=O)[C@@H]1CC[C@@H](C)[C@H]1C1(O)OC(=O)C=C1C(C)C</smiles>

6-Hydroxy-alpinolide 113<smiles>C=C(C)C(CCC(C)=O)CC1=C(C)CCC1=O</smiles>

Alpinolide 114
(+)-Mandassidion 115<smiles>COC(=O)CCC(/C=C1\C(=O)CC[C@@]1(C)O)C(C)C</smiles>

Mandassion A

116

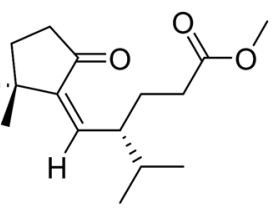

Mandassion B

117<smiles>C=C1CC[C@H](O)/C=C\C=C/C1C</smiles>

Caryophyllenol-I

119<smiles>C=C1CC[C@H](O)/C=C\C[C@H]2C[C@H]1C2(C)C</smiles>
Caryophyllenol-II

120

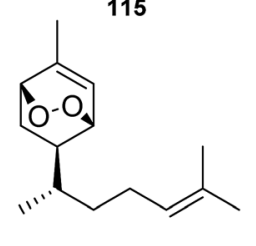

$(1 S, 4 R, 6 R)-1,4-E p i d i o x y-$ bisabola-2,10-diene

121

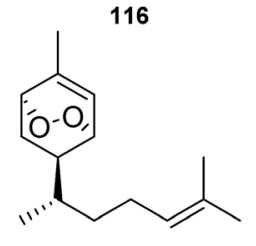

$(1 R, 4 S, 6 R)-1,4$-Epidioxy 3-Hydroxy,11-hydroperoxy-bisabola-2,10-diene

122

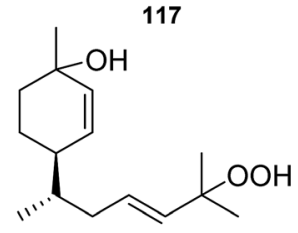

bisabola-1,9-diene<smiles>C=C(C)C(CCC(O)C1C=CC(C)(O)CC1)OO</smiles>

3-Hydroxy-10-hydroperoxybisabola-1,10-diene 124<smiles>C=C1C=C[C@@H](C(C)C/C=C/C(C)(C)O)C[C@H]1O</smiles>

4-Hydroxy-11-hydroperoxy-
bisabola-1,3(15),9-triene 125<smiles>CC(C)=CCC[C@@H](C)[C@H]1C=C[C@@](C)(O)[C@H](O)C1</smiles>

3,4-Dihydroxy-bisabola1,10-diene 126<smiles>CC(=O)C[C@H]1C[C@@H]([C@@H](C)CCC=C(C)C)CC(=O)O1</smiles>

3,4-Seco-biasbol-10-
ene-3-one-1,4-olide
127<smiles>C=C(C)[C@H](O)[C@H](O)CC[C@@H](C)[C@H]1CC(=O)C(C)=C[C@H]1C</smiles>

$(1 S, 6 S)-1 \alpha, 10-$ dihydroxy-biasbol2,11-dienes-4-one

128
(1S,6S)-1 $\alpha$-Hydroxy-

biasbol-2,10-diene-14-al

129

Fig. 2 Sesquiterpenoids from Alpinia species (continued).

indicating 151 might be a potential candidate as a future antidiabetic drug. ${ }^{70}$ A. formosana, A. calcarata Rosc., and A. pahangensis Ridley provided (E)-labda-8(17),12-diene-15-ol-16-al
(152), ${ }^{\mathbf{8 1 , 8 5 , 9 6}}$ while (E)-labda-8(17),13-dien-15-al (153) was only obtained from A. pahangensis Ridley. ${ }^{96}$ Flowers of A. chinensis Rosc. provided compounds 154-161. ${ }^{81,85,97} A$. tonkinensis 

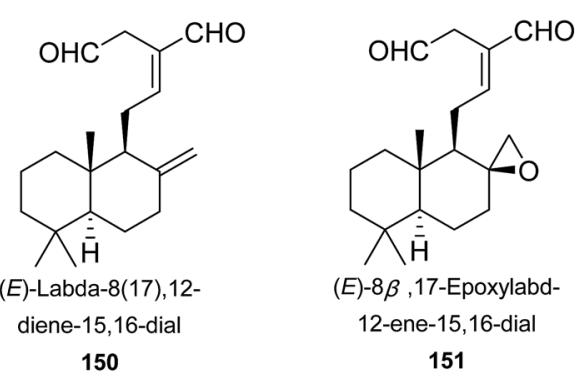

(E)-8 8 ,17-Epoxylabd12-ene-15,16-dial

151<smiles>C=C1CC[C@H]2C(C)(C)CCC[C@]2(C)[C@H]1C/C=C(/C=O)C(O)CO</smiles>

$(E)-14 \xi, 15$-Dihydroxylabda8(17),12-dien-16-al

156<smiles>C=C1CC[C@H]2C(C)(C)CCC[C@]2(C)[C@H]1C[C@H](O)C1=CC(O)OC1=O</smiles>

Zerumin B

163

8(17),12-labdadiene

162<smiles>C=C1CC[C@H]2C(C)(C)CCC[C@]2(C)[C@H]1CC=C(C)CC(=O)O</smiles>

Calcaratarin B

167<smiles>C=C1CC[C@H]2C(C)(C)CCC[C@]2(C)[C@H]1/C=C/c1ccoc1</smiles>

Coronarin $\mathrm{E}$

172<smiles>C=C1CC[C@H]2C(Cl)(Cl)CCC[C@]2(C)[C@H]1[C@H](O)CC1=CCOC1=O</smiles>

168<smiles>C=C1C(O)C[C@H]2C(C)(C)CCC[C@]2(C)[C@H]1/C=C/C1=CCOC1=O</smiles>

Hedyforrestin B

173

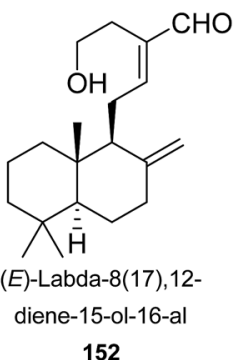<smiles>C=C1CC[C@H]2C(C)(C)CCC[C@]2(C)[C@H]1CC=C(C)CC=O</smiles>

$(E)$-Labda-8(17),13-dien-15-al

153<smiles>C=C1CC[C@H]2C(C)(C)CCC[C@]2(C)[C@H]1CC(O)/C(C=O)=C\CO</smiles>

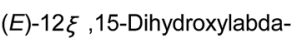

8(17)-13-dien-16-al

$157 / 158$<smiles>C=C1CC[C@H]2C(C)(C)CCC[C@]2(C)[C@H]1/C=C/C1=CCO[C@@H]1O</smiles>

(E)-15,16-Epoxylabda8(17),11,13-trien-16-ol

164<smiles>C=C1CC[C@H]2C(C)(C)CCC[C@]2(C)[C@H]1C/C=C1\C(=O)OC[C@H]1O</smiles>

169

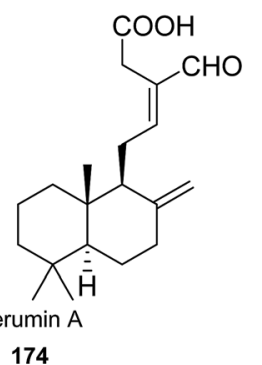<smiles>C=C1CC[C@H]2C(C)(C)CCC[C@]2(C)[C@H]1/C=C/C1=CC(O)OC1=O</smiles>

(E)-15-Hydroxylabda8(17),11,13-trien-16,15-olide

165<smiles>C=C1CCC2C(C)(C)CCCC2(C)[C@H]1/C=C/C1=CCOC1=O</smiles>

Labda-8(17),11,13-trien-15(16)-olide

170

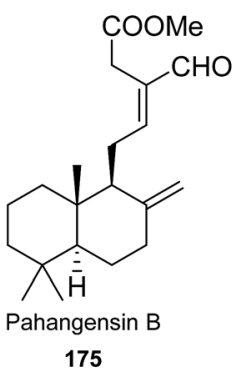<smiles>C=C1CC[C@H]2C(C)(C)CCC[C@]2(C)[C@H]1C/C=C(/C=O)CC(OC)OC</smiles>

Calcaratarin A

166<smiles>C=C1[C@H](O)CC2C(C)(C)CCC[C@]2(C)[C@H]1/C=C/c1ccoc1</smiles>

171

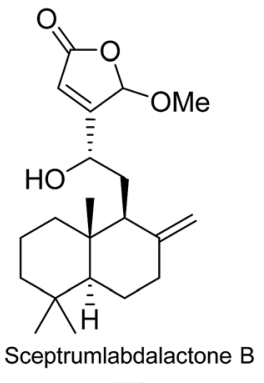

176

Fig. 3 Diterpenoids from Alpinia species.

Gagnep. and A. speciosa K. Schum. (the accepted name is $A$. zerumbet (Pers.) B. L. Burtt \& R. M. Sm.) were the sources of (E)15-nor-16-oxo-8(17),12-labdadiene (162). ${ }^{51,98}$ Both A. zerumbet
(Pers.) Burtt and P. M. Smith and A. pahangensis Ridley gave birth to zerumin B (163). ${ }^{96,99}$ (11E)-15,16-Epoxylabda-8(17),11,13-trien16-ol (164) and (E)-15-hydroxylabda-8(17),11,13-trien-16,15-olide 
<smiles>CC1(C)CCC[C@]2(C)[C@H]1CC[C@]1(CO1)[C@@H]2CC=C1CCOC1=O</smiles>

Galanolactone 177<smiles>C=C1CC[C@H]2C(C)(C)CCC[C@]2(C)[C@H]1CC=C1C(=O)OC[C@@H]1O</smiles>

Isocoronarin D

178<smiles>CC1(C)CCC[C@]2(C)C(/C=C/C3=CC(=O)OC3)C(C=O)=C(O)C(=O)[C@H]12</smiles><smiles>C=C1CC[C@H]2C(C)(C)CCC[C@]2(C)[C@H]1CCC1=CC(=O)OC1</smiles><smiles>C=C1CC[C@H]2C(C)(C)CCC[C@]2(C)[C@H]1Cc1coccc1=O</smiles>

Galaganin 179<smiles>C=C1CC[C@H]2C(C)(C)CCC[C@]2(C)[C@H]1/C=C(/CO)[C@@H](O)CO</smiles>

\section{5,16-olide}

Ottensinin

181

EtO<smiles>CC1(C)CCC[C@]2(C)[C@@H](CC=C3CCOC3=O)C(=O)CC[C@@]12C</smiles>

Methyl (11E)-14,15,16-Trinorlabda-8(17),11-dien-13-oate 182<smiles>C=C1CC[C@H]2C(C)(C)CCC[C@]2(C)[C@H]1C/C=C1\C[C@H](OC)OC1=O</smiles>

Coronarin D methyl ether 186

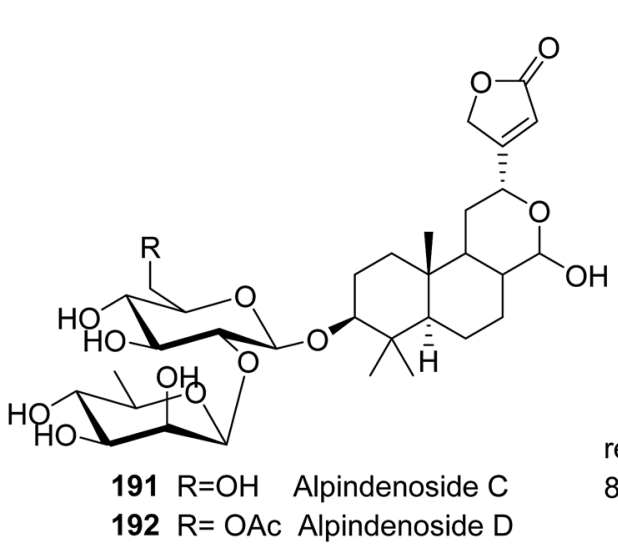<smiles>C[C@H]1CCC[C@@]2(C)[C@@H]3CC=C(C=O)C[C@H](O)CC3CC[C@H]12</smiles>

(12E)-17-Norlabd-12-en8-one-16,15-olide

183<smiles>CC1(C)CCC[C@]2(C)[C@@H](C/C=C3\C=COC3=O)[C@H](C(=O)O)CC[C@]12C</smiles>

$(12 Z, 14 R)-L a b d a-8(17), 12-$ diene-14,15,16-triol

184<smiles>C=C1CC[C@H]2C(C)(C)CCC[C@]2(C)[C@H]1C[C@H](O)C1=C[C@@H](OCC)OC1=O</smiles>

\section{(12R)-15-Ethoxy-12-hydroxylabda-} 8(17),13(14)-dien-15,16-olide 185

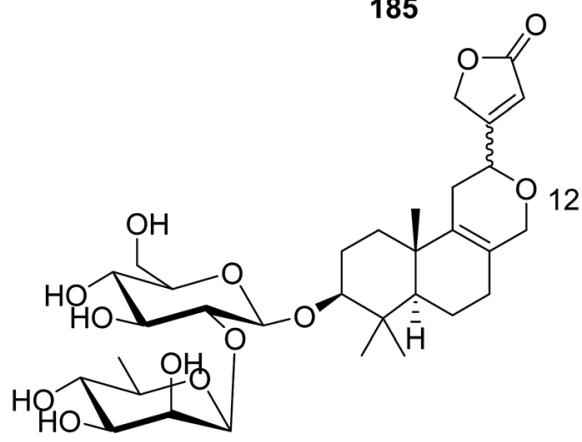

(E)-Labda-12,14-dien-15(16)olide-17-oic acid 188<smiles>COc1cc(OC)c([C@H]2C=CCC[C@]23C(=O)C[C@H]2C(C)(C)CCC[C@]2(C)[C@H]3CC=C2CCOC2=O)cc1OC</smiles>

rel-Labd-12-en-15(16)-olid-7-one8R-spiro-1'-[2S-(2,4,5-trimethoxyphenyl)-3-cyclohexene]

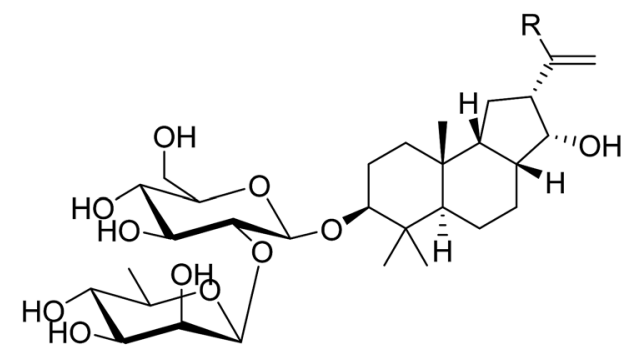

$194 \mathrm{R}=\mathrm{CH}_{2} \mathrm{OH}$ Noralpindenoside $\mathrm{A}$

$195 \mathrm{R}=\mathrm{Me}$ Noralpindenoside B
189 (12S) Alpindenoside A 190 (12R) Alpindenoside $\mathrm{B}$

\section{3}

Fig. 3 Diterpenoids from Alpinia species (continued).

(165) were found in the flowers of $A$. chinensis Rosc. ${ }^{97}$ It is noteworthy that 164 was actually a mixture of two epimers. Rhizomes of $A$. calcarata Rosc. produced calcaratarins A-D (166-169) and labda-8(17),11,13-trien-15(16)-olide (170). ${ }^{85}$ Rhizomes of $A$. malaccensis yielded coronarin A (171), coronarin E (172), and hedyforrestin B (173). ${ }^{100}$ Coronarin E (172) was also isolated from $A$. 
<smiles>C=C1CCC[C@]2(C)[C@H]1CCC(=C)[C@@H]2C1C=C(C=O)[C@@H](CO)OO1</smiles>

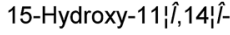
peroxylabda-8(17),12-dien-16-al 196/197

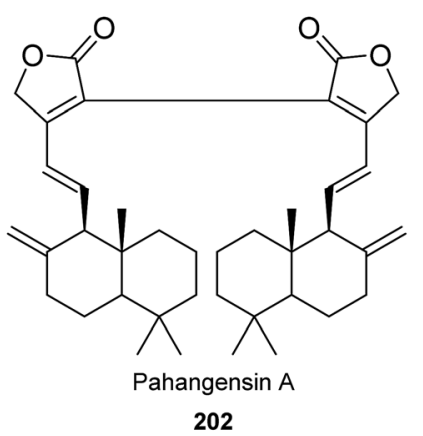<smiles>CC1(C)CCC[C@]2(C)[C@H]1CC[C@]1(C)[C@@H](O)CC(C=O)=CC[C@H]12</smiles>

Galanal A

199<smiles>C=C1CC(C)(C)[C@@H]2CCCC[C@]2(C)/C1=C\C[C@@H]1C(=C)CCC2(C)C(C)(C)CCC[C@]12C</smiles>

Pahangensin $\mathrm{A}$ 203<smiles>CC1(C)CCC[C@]2(C)[C@H]1CC[C@]1(C)[C@@H](O)CC(C=O)=CC[C@@H]12</smiles>
200<smiles>COc1cc(O)c(/C=C/C(C=O)=C/C[C@@H]2[C@]3(C)CCCC(C)(C)[C@@]3(C)CC[C@]23CO3)c(O)c1C(=O)/C=C/c1ccccc1</smiles>

A mixed metabolite 201

Fig. 3 Diterpenoids from Alpinia species (continued).

zerumbet (Pers.) Burtt and P. M. Smith, and A. chinensis Rosc. ${ }^{97,99,100}$ Three antibacterial constituents, zerumin A (174), pahangensin B (175), and sceptrumlabdalactone B (176), were isolated from A. pahangensis Ridley. ${ }^{96}$ Interestingly, zerumin A (174) was also obtained from A. calcarata Rosc. and A. zerumbet (Pers.) Burtt and P. M. Smith. ${ }^{85,99}$ Compound 175 was also found in A. japonica (Thunb.) Miq., with NO production inhibition $\left(\mathrm{IC}_{50}\right.$ $=34.3 \mu \mathrm{M})$ in LPS-induced RAW264.7 macrophages. $^{101} \mathrm{Gal}$ anolactone (177) was isolated from A. katsumadai Hayata and $A$. galanga. It was reported to have moderate antifungal activity to Candida guilliermondii PW44 and Candida tropicalis PW30 with both MIC values of $25 \mu \mathrm{g} \mathrm{mL}{ }^{-1} \cdot{ }^{93}$ Isocoronarin D (178) was found in A. galanga (L.) Willd and A. calcarata Rosc., which weakly suppressed the proliferation of four cancer cells lines of HeLa, A549, HepG2, and SMMC-7721 in a concentration-dependent way with $\mathrm{IC}_{50}$ values ranging from 69.1 to $87.0 \mu \mathrm{g} \mathrm{mL} \mathrm{m}^{-1}{ }^{64,67}$ Seeds of $A$. galanga yielded galaganin (179), which showed moderate cytotoxicity towards DU145, MCF-7, H522, and k562 cells with $\mathrm{IC}_{50}$ values of $8.2,13.8,17.8$, and $16.1 \mu \mathrm{M}$, respectively. ${ }^{102}$ Rhizomes of $A$. pinnanensis T. L. Wu et Senjen produced labda-8(17),13(14)-di-en-15,16-olide (180) and ottensinin (181). ${ }^{96}$ A. japonica provided compounds 182-187, of which 182 and 183 were norlabdanes. ${ }^{101}$ Compounds 182, 185, and 186 exhibited

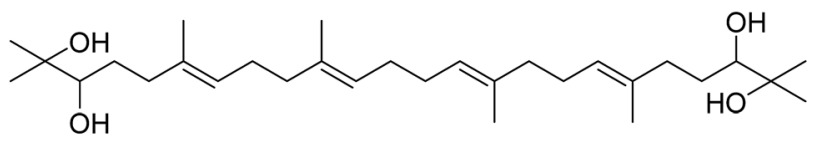

2,3,22,23-Tetrahydroxyl-2,6,10,15,19,23-hexamethyl-6,10,14,18-tetracosatetraene 207

Fig. 4 Triterpenoids from Alpinia species. significant NO production inhibitory effects in LPS-induced RAW264.7 macrophages, with respective $\mathrm{IC}_{50}$ values of 25.9, 14.6, and $25.6 \mu \mathrm{M}$, compare to $39.6 \mu \mathrm{M}$ of the positive control, $N$ monomethyl-L-arginine (L-NMMA). ${ }^{101}$ Ethanol extract of $A$. oxyphylla Miq. provided 188, which showed moderate hypoglycemic effect with inhibitory rates of $10.0 \%$ at $60 \mu \mathrm{M} .^{77}$ Ottensinin showed moderate antibacterial activity on the Gram-positive bacteria of Bacillus cereus with MIC value of $0.25 \mu \mathrm{g} \mu \mathrm{L}^{-1} .^{96}$ Alpindenosides A-D (189-192) were four labdane glycosides from A. densespicata Hayata. They didn't show cytotoxic activities against four human tumor cell lines of Hela, KB, Doay, and WiDr at $20 \mu \mathrm{M}$. Instead, they all exhibited moderate NO inhibitory activities with $\mathrm{IC}_{50}$ ranging from 30 to $49 \mu \mathrm{M} \cdot{ }^{103}$ Leaves of $A$. flabellate provided rel-labda-12-en-15(16)-olid-7-one-8R-spiro-1' [2S-(2,4,5-trimethoxyphenyl)-3-cyclohexene] (193), a unique labdane diterpene coupled with a phenylbutenoid. ${ }^{\mathbf{1 0}}$ Noralpindenosides A (194) and B (195) were two norditerpene glycosides from $A$. densespicata Hayata, both of which showed moderate inhibitory effects on NO production with $\mathrm{IC}_{50}$ values of 34.2 and $49.3 \mu \mathrm{M}$, respectively. ${ }^{103} \quad(E, E)$-15-Hydroxylabda8(17),11,13-trien-16-al (196) and its diastereoisomer (197) from A. chinensis Rosc. may arise by direct oxygenation of $(E, E)-15-$ hydroxylabda-8(17),11,13-trien-16-al. ${ }^{97}$ From flowers of $A$. chinensis Rosc., coronarin B (198) containing a seven-membered endoperoxide hemiacetal was isolated. ${ }^{97}$ It should be noted that although the structure and its NMR and MS spectroscopic data referred to coronarin B (CAS number: 119188-38-4) in the reference, the author gave a wrong name for this compound as coronarin C (CAS number: 119188-35-1) which was previously isolated from Hedychium coronarium. ${ }^{105}$ Galanals A (199) and B (200) were obtained from A. galanga (L.) Willd. Both compounds showed significant antifungal activities against 


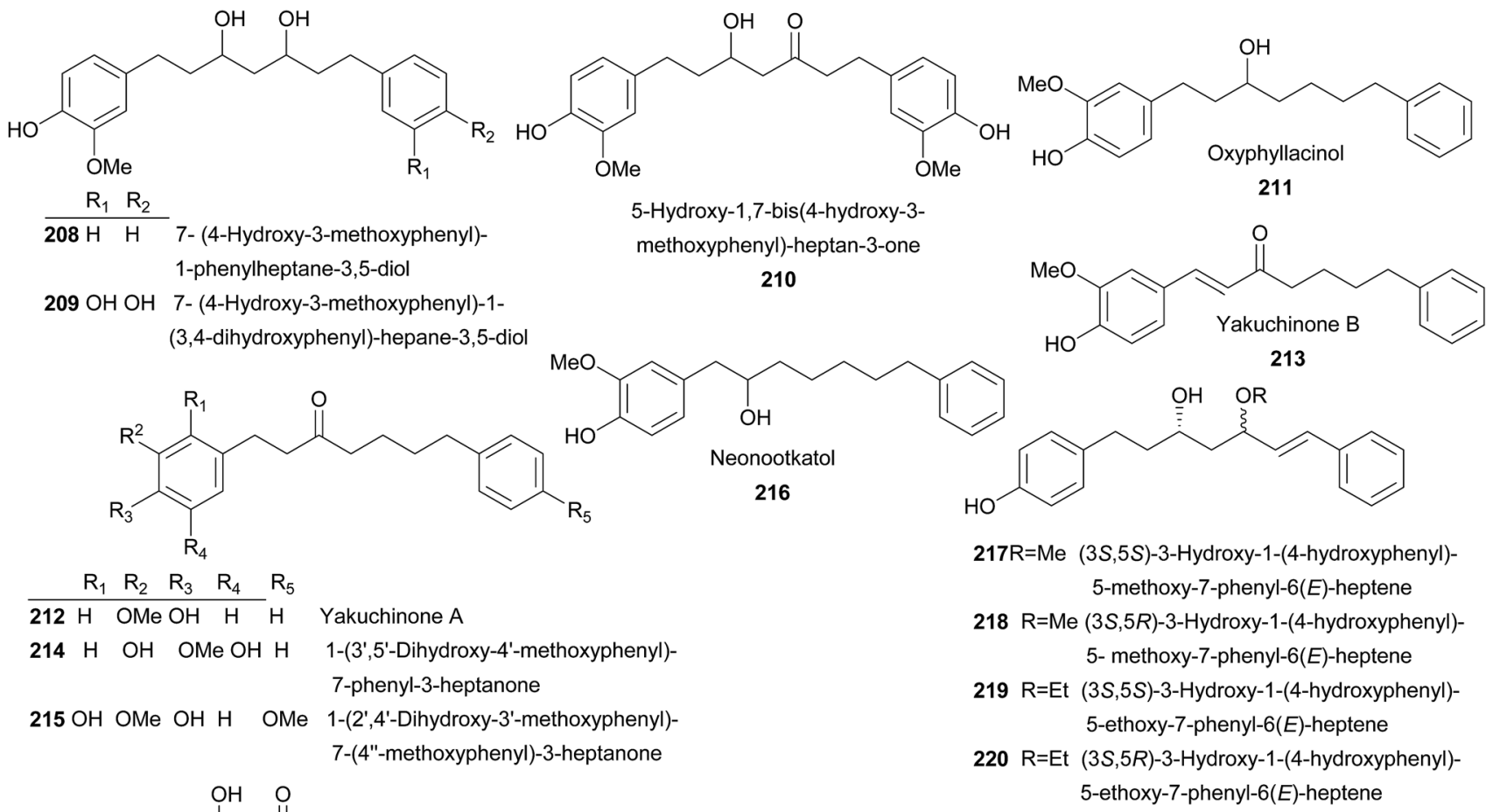<smiles>CO[C@H](CCc1ccc(O)cc1)CC(=O)/C=C/c1ccc(O)cc1</smiles>
1,3,6-heptatrien-5-one 221<smiles>[R]C(C=Cc1ccc([R3])cc1)=CC(=O)CCc1ccc([R])cc1</smiles>

$\begin{array}{llll}R_{1} & R_{2} & R_{3}\end{array}$

$222 \mathrm{OH} \mathrm{H \quad OH}$ 1,7-Bis(4-hydroxyphenyl)-hepta-4E,6E-dien-3-one $223 \mathrm{OH} \mathrm{OH} \mathrm{OH} \mathrm{1,7-Bis(4-hydroxyphenyl)-3-hydroxy-1,3-heptadien-5-one}$ 229 H H H Alnustone

$230 \mathrm{H} \mathrm{OH} \mathrm{H}$ (4Z,6E)-5-Hydroxy-1,7-diphenyl-4,6-heptadien-3-one<smiles>Oc1ccc(CCC(O)CCc2ccc(O)cc2)cc1</smiles>

225 1,7-diphenyl-1-heptene 226<smiles>O[C@H](C=Cc1ccccc1)C[C@@H](O)CCc1ccccc1</smiles>

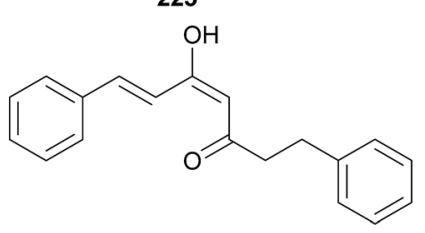

(E,E)-5-Hydroxy-1,7-diphenyl-4,6-heptadien-3-one 227

Fig. 5 Diarylheptanoids from Alpinia species.

Candida guilliermondii PW44 with MIC values of $12.5 \mu \mathrm{g} \mathrm{mL}{ }^{-1}$. Furthermore, galanal A exhibited potent cytotoxic activity against $\mathrm{KB}$ cells $\left(\mathrm{IC}_{50}=3.25 \mu \mathrm{g} \mathrm{mL} \mathrm{m}^{-1}\right) \cdot{ }^{7,93}$ Compound 201 was a novel metabolite conjugated of labdane diterpene with chalcone from aerial parts of A. katsumadai Hayata. ${ }^{49}$ A. pahangensis Ridley provided pahangensins A (202) and $\mathrm{C}$ (203) as antibacterial constituents. ${ }^{96,106}$ A . pahangensis Ridley produced calcaratarins D (204) and $\mathrm{E}$ (205), both of which were cytotoxic against human $\mathrm{KB}$ cells in vitro with $\mathrm{IC}_{50}$ value of 0.21 and $0.15 \mu \mathrm{g} \mathrm{mL}{ }^{-1}$, respectively ${ }^{\mathbf{1 0 7}}$ From seeds of A. katsumadai Hayata, a grayanane diterpenoid was isolated and characterized as rhodomollein I (206). ${ }^{\mathbf{1 0 8}}$

\subsection{Triterpenoids}

Up to now, only one triterpene was found from this genus (Fig. 4). It was named as 2,3,22,23-tetrahydroxyl-2,6,10,15,19,23-hexamethyl6,10,14,18-tetracosatetraene (207), an acyclic triterpenoid, isolated from the seeds of $A$. katsumadai L. $^{109}$ It showed weak cholesterol acyltransferase inhibitory activity with $\mathrm{IC}_{50}$ value of $47.9 \mu \mathrm{M.} .^{109}$

\section{Diarylheptanoids}

A total of 143 diarylheptanoids (208-350, Fig. 5) were isolated from Alpinia species, including 66 acyclic diarylheptanoids (208-273), 11 cyclic diarylheptanoids (274-284), 50 diarylheptanoid and flavonoid conjugates (285-334), 10 dimeric diarylheptanoids (335-344), and six others (345-350).

Compounds 208-210 were isolated from rhizomes of $A$. officinarum Hance. They were moderate or weak NO production inhibitors. ${ }^{110}$ From fruits of A. oxyphylla, oxyphyllacinol (211) and yakuchinones A-B (212-213) were isolated, of which 211 was a NO production inhibitor, while 212 and 213 exhibited anti-tumor activities to human promyelocytic leukemia (HL-60) cells in a concentration-related manner. ${ }^{32,67}$ In addition, 212 
<smiles>O[C@H](CC/C=C/c1ccccc1)CCc1ccccc1</smiles>

(S)-1,7-Diphenyl-6(E)-hepten-3-ol

228<smiles>O=C(CCc1ccc(O)cc1)C[C@H](O)/C=C/c1ccccc1</smiles>

5-Hydroxy-1-(4'-hydroxyphenl)7-phenyl-hepta-6-en-3-one

233<smiles>OC(CC/C=C/c1ccccc1)CCc1ccccc1</smiles>

trans-1,7-Diphenyl-5-hydroxy1-heptene 238<smiles>O=C(CCc1ccccc1)CC(O)/C=C/c1ccccc1</smiles>

1,7-Diphenyl-5-hydroxy-6-hepten-3-one

241<smiles>[R]c1ccc(CCC(=O)C[C@H](O)CCc2ccc(O)c([R5])c2)cc1[R]</smiles><smiles>[R3]c1ccc(/C=C/C([R])C[C@@H](O)CCc2ccc([R])cc2)cc1</smiles>

\begin{tabular}{rll}
$\mathrm{R}_{1}$ & $\mathrm{R}_{2}$ & $\mathrm{R}_{3}$ \\
\hline $234 \mathrm{H}$ & $\mathrm{O}$ & $\mathrm{OH}$ \\
$(-)-(R)-4 "$ Hydroxyyashabushiketol
\end{tabular} $235 \mathrm{OH} \mathrm{OH} \mathrm{H}$ (3S,5S)-Alpinikatin<smiles>O=C(/C=C/C=C/c1ccc(O)cc1)/C=C/c1ccc(O)cc1</smiles>

1,7-bis(4-Hydroxyphenyl)-1,4,6-heptatrien-3-one 239<smiles>[Z16][C@H](O)/C=C/c1ccccc1</smiles>

241a $S$

241b $R$<smiles>O=C(CCc1ccccc1)C[C@H](O)/C=C/c1ccccc1</smiles>

(5R,6E)-1,7-Diphenyl-5hydroxyhept-6-en-3-one

232<smiles>[R12]OC(CCc1ccc(O)cc1)CC([R20])/C=C/c1ccccc1</smiles><smiles>O=C(/C=C/c1ccc(O)cc1)CC(=O)/C=C/c1ccc(O)cc1</smiles>

240<smiles>CCOC(CCc1ccc(O)c(OC)c1)CC(=O)ON=C1OCCOCCOCCO1</smiles>

242<smiles>[R17]OC(CCc1ccc(O)c([R2])c1)CC(=O)CCc1ccccc1</smiles>

$250 \mathrm{Me} \mathrm{H}$ 5(S)-7-(4-Hydroxyphenyl)-5-methoxy1-phenylheptan-3-one

251 Et OMe 5(S)-Ethoxyl-7-(4-hydroxy-3-methoxyphenyl)- 1-phenyl-3-hepta none

\begin{abstract}
$\frac{\mathrm{R}_{1} \mathrm{R}_{2} \mathrm{R}_{3}}{243 \mathrm{H} \mathrm{OH} \mathrm{OMe}}$ 5-Hydroxy-7-(4-hydroxy-3- methoxyphenyl)1-(4-hydroxyphenyl)-3-heptanone

$244 \mathrm{OMe} \mathrm{OHH}$ 5-Hydroxy-1-(4-hydroxy-3-methoxyphenyl)7-(4-hydroxyphenyl)-3-heptanone
\end{abstract}<smiles>[R]c1ccc(CCC(=O)CC(O)CCc2cc([R])c(O)c([R])c2)cc1[R]</smiles><smiles>[R]c1ccc(CCC(=O)C[C@@H]([R6])CCc2cc([R2])c(O)c([R6])c2)cc1[R]</smiles>

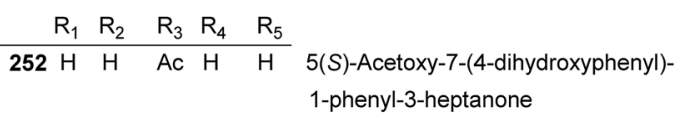

$253 \mathrm{OH} \mathrm{OMe} \mathrm{H} \mathrm{OMe} \mathrm{OH} \mathrm{(5R)-5-Hydroxy-1-(4-hydroxy-3-}$ methoxyphenyl)-7-(4,5-dihydroxy-<smiles>O=C(CCCCc1ccc(O)cc1)CCc1ccccc1</smiles>

7-(4-Hydroxyphenyl)-1-phenyl-3-heptanone

254

Fig. 5 Diarylheptanoids from Alpinia species (continued).

also possessed insecticidal, ${ }^{36}$ anti-adipocyte differentiation, ${ }^{111}$ NO production inhibitory, ${ }^{46}$ and cardiotonic activities. ${ }^{112}$ Compounds 213-216 were also yielded by fruits of $A$. oxyphylla. ${ }^{113,114}$ Seeds of $A$. blepharocalyx K. Schum. gave birth to 217-225. ${ }^{115-117}$ Among these compounds, 1,7-bis(4-hydroxyphenyl)-3-hydroxy-1,3-heptadien-5one (223) significantly inhibited platelet aggregation induced by 
<smiles>[R]c1ccc(CCC(=O)/C=C/CCc2cc([R3])c(P)c(P)c2)cc1[R]</smiles>
$255 \mathrm{OH} \mathrm{H} \quad \mathrm{OMe} \mathrm{OH} \quad \mathrm{H}$ 1-(4-Hydroxyphenyl)-7-(4-hydroxy- 3-methoxyphenyl)-4E-en-3-heptanone $256 \mathrm{H} \quad \mathrm{H} \quad \mathrm{H} \quad \mathrm{OH}$ OMe (E)-7-(4-Hydroxy-3-methoxyphenyl)-1-phenylhept-4-en-3-one $257 \mathrm{OH} \mathrm{OMe} \mathrm{OH} \mathrm{OH} \mathrm{H} \mathrm{7-(3,4-Dihydroxyphenyl)-1-(4-hydroxy-3-methoxyphenyl)-4-en-3-heptanone}$ $259 \mathrm{H} \quad \mathrm{H} \quad \mathrm{OMe} \quad \mathrm{OH} \quad \mathrm{OH}$ 7-(4",5"-Dihydroxy-3"-methoxyphenyl)-1-phenyl-4-heptene-3-one $263 \mathrm{H} \quad \mathrm{H} \quad \mathrm{H} \quad \mathrm{H} \quad \mathrm{H} \quad$ AO-5<smiles>COc1cc(CCC(=O)/C=C(O)/C=C/c2ccccc2)ccc1O</smiles>
$269 \mathrm{H} \quad \mathrm{H} \quad \mathrm{OMe} \mathrm{OH} \quad \mathrm{H} \quad \mathrm{AO}-4$ $271 \mathrm{H} \quad \mathrm{H} \quad \mathrm{H} \quad \mathrm{OH} \quad \mathrm{H} \quad \mathrm{AO}-3$<smiles>O=C(C/C=C/Cc1ccccc1)CCc1ccccc1</smiles><smiles>[R][R]</smiles>

(4E,6E)-5-Hydroxy-1-(4-hydroxy-3methoxyphenyl)-7-phenylhepta-4,6-dien-3-one 258

1,7-Diphenyl-5-heptene-3-one 261
$260 \mathrm{Me} \mathrm{OH} \mathrm{H} \mathrm{5-Methoxy-7-(4"} \mathrm{-hydroxyphenyl)-}$ 1-phenyl-3-heptanone

$265 \mathrm{H} \mathrm{OH} \mathrm{H} \mathrm{5-Hydroxy-7-(4"} \mathrm{-hydroxyphenyl)-}$ 1-phenyl-3-heptanone<smiles>[R]c1ccc(CCC(=O)CC(=O)CCc2ccccc2)cc1[R2]</smiles><smiles>[R]OC(=O)CC(CCc1ccc([R2])c([R3])c1)O[R17]</smiles>

$$
\begin{array}{rrrr} 
& \mathrm{R}_{1} & \mathrm{R}_{2} & \mathrm{R}_{3} \\
\hline 264 & \mathrm{H} & \mathrm{H} & \mathrm{H}
\end{array}
$$
$266 \mathrm{H} \quad \mathrm{OH}$ OMe AO-1 $267 \mathrm{Me} \mathrm{OH} O M e$ AO-2<smiles>[R]COC(CCc1ccc([R])c([R3])c1)CC(=O)CCc1ccccc1</smiles>
2680H OM 7-(4"-Hydroxy-3"-methoxyphenyl)1-phenyl-3,5-heptadione<smiles>O=C(/C=C/C(O)Cc1ccccc1)CCc1ccccc1</smiles>

6-Hydroxy-1,7-diphenyl-4-en-3-heptanone 270<smiles>COC(CCc1ccccc1)CC(=O)CCc1ccccc1</smiles>

(5S)-5-Methoxy-1,7-diphenyl-3-heptanone 272<smiles>Oc1ccc(CC[C@@H]2CC=C[C@H](c3ccc(O)cc3)O2)cc1</smiles>

(3S,7R)-5,6-Dehydro-1,7-bis(4-hydroxyphenyl)4"-de-O-methylcentrolobine

$$
\mathrm{HO}
$$
274<smiles>[R1][C@H]1CCO[C@H](c2ccc(O)cc2)[C@@H]1[R2]</smiles><smiles>CCO</smiles><smiles>Oc1ccc(CC[C@@H]2CC=C[C@H](c3ccc(O)cc3)O2)cc1</smiles>

(3S,7S)-5,6-Dehydro-4"-deO-methylcentrolobine 283<smiles>Oc1ccc(CC[C@@H](O)C[C@@H](O)CCc2ccccc2)cc1</smiles>

273

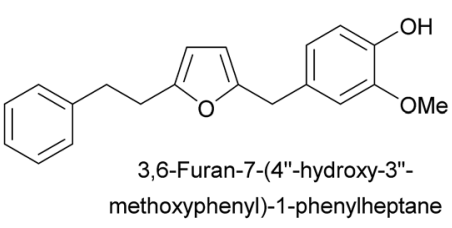

284

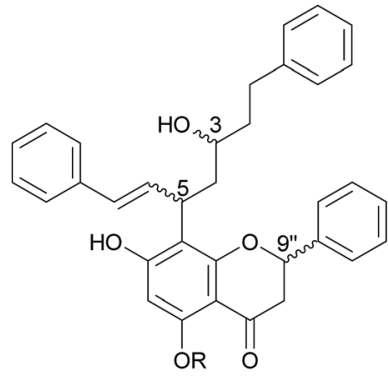

R 35 9"

285 Me $S R R$ Calyxin $N$ $286 \mathrm{Me} S R S$ Calyxin $O$ 287 Me $R S R$ ent-Calyxin $O$ 288 Me $R S S$ ent-Calyxin $N$ $289 \mathrm{H} S S \quad R \quad S$ Calyxin $\mathrm{P}$ $290 \mathrm{H} S R \quad R \quad 9$ "-Epicalyxin $\mathrm{P}$<smiles>COc1cc2c(c3c1C(=O)C[C@@H](c1ccccc1)O3)[C@@H](CC(=O)CCc1ccccc1)C[C](c1ccccc1)O2</smiles>

$2915 S$ Calyxin $S$ $2925 R$ 5-Epicalyxin $S$

Fig. 5 Diarylheptanoids from Alpinia species (continued).

collagen with $\mathrm{IC}_{50}$ value of $14.7 \mu \mathrm{g} \mathrm{mL}^{-1} \cdot{ }^{117}(3 S, 6 E)$-Methoxy1,7-bis(4-hydroxyphenyl)-6-hepten-5-one (224) and (3S,5S)3,5-dihydroxy-1,7-bis(4-hydroxyphenyl)heptane (225) showed significant antiproliferative activities against murine colon 26L5 carcinoma and human HT-1080 fibrosarcoma with $\mathrm{IC}_{50}$ values of 5.2 and $12.8 \mu \mathrm{M}$, respectively. ${ }^{115,116}$ Both $A$. pinnanensis 


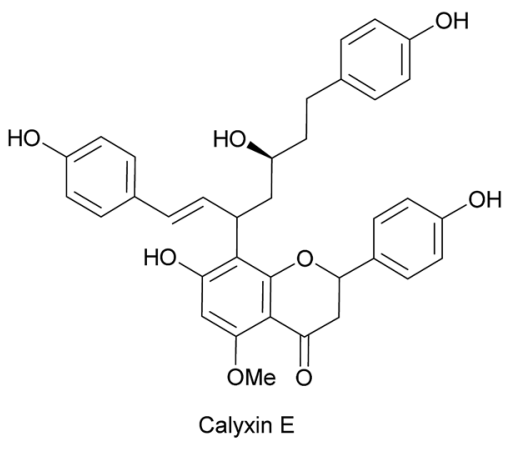

293<smiles>COc1cc2c(c3c1C(=O)C[C@@H](c1ccc(O)cc1)O3)[C@@H]1C[C@H](CCc3ccc(O)cc3)O[C@H](c3ccc(O)cc3)[C@H]1[C@@H](c1ccc(O)cc1)O2</smiles>

298 Calyxin J 299 Epicalyxin J<smiles>[R]c1ccc(/C=C/C(=O)c2c(OC)cc(O)c([C@H](/C=C/c3ccccc3)C[C@H](O)CCc3ccc([R2])cc3)c2O)cc1</smiles>
$\frac{\mathrm{R}_{1} \quad \mathrm{R}_{2}}{306 \mathrm{OH} \quad \mathrm{H} \text { Calyxin Q }}$ $307 \mathrm{H} \quad \mathrm{OH}$ Calyxin R<smiles>COc1cc(O)c([C@H]2C[C@H](CCc3ccccc3)O[C@H](c3ccc(O)cc3)C2)c(O)c1C(=O)/C=C/c1ccc(O)cc1</smiles>

313<smiles>COc1cc(O)c([C@H]2CC(C(C)C)O[C@@H](c3ccc(O)cc3)C2)c2c1C(=O)C[C@@H](c1ccc(O)cc1)O2</smiles>

294 Calyxin $G$ 295 Epicalyxin G

$\mathrm{HO}$<smiles>COc1cc(O)c([C@H]2C[C@H](CCc3ccc(F)cc3)O[C@H](c3ccc(O)cc3)C2)c2c1C(=O)C[C@@H](c1ccc(O)cc1)O2</smiles>

300 Calyxin K 301 Epicalyxin $K$<smiles>[R]c1ccc(CC[C@@H](O)C/C=C/[C@H](C(C(=O)O)=C(O)O)c2ccc(O)cc2)cc1</smiles>
\begin{tabular}{llllll} 
& $\mathrm{R}_{1}$ & $\mathrm{R}_{2}$ & 3 & 7 & \\
\hline 308 & $\mathrm{OH}$ & $\mathrm{H}$ & $R$ & $R$ & Katsumain $\mathrm{C}$
\end{tabular} $309 \mathrm{OH} \quad \mathrm{H} \quad R \quad S \quad 7$-epi-Katsumain C $310 \mathrm{H} \quad \mathrm{H} \quad R \quad R$ ent-Alpinnanin $\mathrm{B}$ $311 \mathrm{H} \quad \mathrm{H} \quad S \quad R$ ent-Alpinnanin A $312 \mathrm{H} \quad \mathrm{OH} \quad R \quad R$ ent-Calyxin $\mathrm{H}$ $319 \mathrm{OH} O H \quad S \quad S$ Calyxin $B$ $320 \mathrm{OH} \quad \mathrm{OH} S \quad R$ Epicalyxin $B$ $321 \mathrm{H} \quad \mathrm{H} \quad S \quad S$ Alpinnanin $B$ $322 \mathrm{H} \quad \mathrm{OH} \quad S \quad R$ Epicalyxin $\mathrm{H}$ $323 \mathrm{H} \quad \mathrm{OH} \quad \mathrm{S} S$ CalyxinH $332 \mathrm{H} \quad \mathrm{H} \quad R \quad S$ Alpinnanin $A$

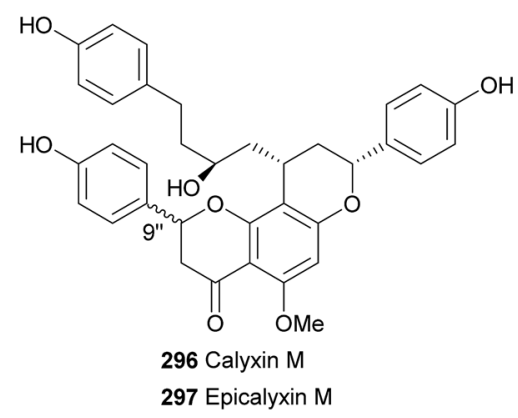

297 Epicalyxin M<smiles>COc1cc(O)c([C](C=CC[C@H](O)CCc2ccc(O)cc2)c2ccc(O)cc2)c2c1C(=O)C[C@@H](c1ccc(O)cc1)O2</smiles>

$3027 S$ Calyxin C 30375 Epicalyxin C $3047 R$ Calyxin $D$ $3057 R$ Epicalyxin $D$ 
<smiles>COc1cc(O)c(C(/C=C/C[C@H](O)CCc2ccccc2)c2ccccc2)c(O)c1C(=O)/C=C/c1ccccc1</smiles>

318<smiles>[R]C(CCc1ccc(O)cc1)C[C@H](/C=C/c1ccc(O)cc1)c1c(O)cc(OC)c(C(=O)/C=C/c2ccccc2)c1O</smiles>

$\begin{array}{lll}R_{1} & R_{2} & R_{3}\end{array}$

$330 \mathrm{OH} \mathrm{OOH} \mathrm{OH}$ Calyxin $\mathrm{A}$

$331 \mathrm{OH} \quad \mathrm{OH} \quad \mathrm{OH}$ Deoxycalyxin $\mathrm{A}$

$333 \mathrm{H} \quad \mathrm{OH} \quad \mathrm{H}$ Alpinnanin $\mathrm{C}$<smiles>O=C(CCC1=CCCC=C1)[C@H]1C(=O)[C@H](Cc2ccccc2)[C@@](O)(CCc2ccccc2)C[C@H]1O</smiles>

335<smiles>COc1cc(O)c2c(c1C(=O)/C=C/c1ccc(O)cc1)O[C@@]1(C)[C@H](c3ccc(O)cc3)O[C@@H](CCc3ccc(O)cc3)C[C@@H](c3ccc(O)cc3)[C@H]21</smiles>

$\mathrm{HO}$<smiles>[R]C1CC[C@@H](CCc2ccc(C)cc2)O[C@]1([CH2-])c1ccc(O)cc1</smiles>

$3247^{\prime \prime \prime \prime} R$ Calyxin I

$3267^{\prime \prime \prime '} S$ Epicalyxin I

$327 \mathrm{R}=\mathrm{H}, 7 \mathrm{~S}$ Epicalyxin $\mathrm{F}$

$328 \mathrm{R}=\mathrm{H}, 7 R \quad$ Calyxins $\mathrm{F}$

$329 \mathrm{R}=\mathrm{OH}, 7 S$ 6-Hydroxycalyxin $\mathrm{F}$<smiles>COc1cc(O)c2c(c1C(=O)/C=C\c1ccc(O)cc1)O[C@@H](c1ccc(O)cc1)C[C@@H]2C[C@H](O)CCc1ccc(O)cc1</smiles>

Calyxin L

325<smiles>COc1cc(CC[C@H](CC(=O)CCc2ccccc2)c2c(O)cc(O)c3c(=O)c(O)c(-c4ccccc4)oc23)ccc1O</smiles>

334<smiles>COc1cc(CCC(=O)CCc2ccccc2)cc(-c2cc(CC[C@H](CC(=O)CCc3ccccc3)OC)cc(OC)c2O)c1O</smiles>

336

337<smiles>COc1cc(CC/C=C/C(=O)CCc2ccccc2)ccc1Oc1cc(CC/C=C/C(=O)CCc2ccccc2)cc(OC)c1O</smiles><smiles>COc1cc(CC/C=C/C(=O)CCc2ccccc2)cc(-c2cc(CC/C=C/C(=O)CCc3ccccc3)cc(OC)c2O)c1</smiles>

340 (1-7) $S$ Blepharocalyxin A

341(1-7) $R$ Blepharocalyxin $B$

Fig. 5 Diarylheptanoids from Alpinia species (continued).

and alnustone (229) were isolated from A. katsumadai Hayata with significantly neuraminidase inhibitory in vitro with $\mathrm{IC}_{50}$ values between 1.0 and $6.1 \mu \mathrm{M} .{ }^{56}$ In addition, 229 also possessed antiemetic, ${ }^{\mathbf{1 2 1}}$ antimycobacterial activities, ${ }^{\mathbf{1 2 0}}$ and significantly inhibited proliferation of Bel 7402 and L0-2 cells. ${ }^{122}$ Investigation of A. katsumadai Hayata also led the isolation of 
$\mathrm{HO}$<smiles>Oc1ccc(/C=C/[C@@H](C[C@H](O)CCc2ccc(O)cc2)[C@H]2[C@@H](O)C[C@@H](CCc3ccc(O)cc3)O[C@H]2c2ccc(O)cc2)cc1</smiles>

342
HO<smiles>COc1cc(O)c([C@@H]2C[C@H](CCc3ccc(F)cc3)O[C@H](c3ccc(O)cc3)[C@H]2C(/C=C/c2ccc(O)cc2)c2ccc(O)cc2)c(O)c1C(=O)/C=C/C[C@H](O)CCc1ccc(O)cc1</smiles>

HO<smiles>Oc1ccc(/C=C/[C@H]2CCO[C@@H]3C[C@H](CCc4ccc(O)cc4)O[C@H](c4ccc(O)cc4)[C@H]23)cc1</smiles><smiles>CCCc1ccc(O)cc1</smiles>

348<smiles>COc1cc2c(c3c1C(=O)C[C@@H](c1ccc(O)cc1)O3)[C@@H]1C[C@@H](CCc3ccc(O)cc3)O[C@H]1O2</smiles><smiles>CC(C)=CCCC1=CCC(C(=O)CCc2ccccc2)C(CCc2ccccc2)C1</smiles>

Officinaruminane B 347<smiles>CCCCCCCCCCC(=O)CCc1ccccc1</smiles>

Katsumadain B

349<smiles>O=C(/C=C/c1ccccc1)C(CCc1ccccc1)C(=O)CCc1ccccc1</smiles>

4-Phenethyl-1,7-diphenyl-1-heptene-3,5-dione 350

Fig. 5 Diarylheptanoids from Alpinia species (continued).

compounds $\quad$ 230-238. ${ }^{\text {49,119,121,123-125 }}$ 1,7-Bis(4-hydroxyphenyl)1,4,6-heptatrien-3-one (239) and bisdemethoxycurcumin (240) were obtained from rhizomes of A. galanga (L.) Willd, both of which significantly inhibited the proliferation of melanoma cells and indistinctively inhibited cellular tyrosinase. ${ }^{\mathbf{1 2 6}}$ A planar structure of 1,7-diphenyl-5-hydroxy-6-hepten-3-one (241) was reported from $A$. nutans Rosc., ${ }^{127}$ A. rafflesiana Wall.ex.Bak., ${ }^{128}$ and $A$. officinarum Hance. ${ }^{129}$ While its enantiomers, $5 S$ (241a) and $5 R(\mathbf{2 4 1 b})$ counterparts, were identified from $A$. mutica Roxb. ${ }^{130}$ and A. katsumadai Hayata, ${ }^{119}$ respectively. It was shown that a large amount of diarylheptanoids (242-276) were obtained from the rhizomes of A. officinarum Hance. ${ }^{27,131-136}$ 7-(3,4Dihydroxyphenyl)-1-(4-hydroxy-3-methoxyphenyl)-4-en-3-heptanone (257) displayed moderate cytotoxicity against human tumor cell lines of HepG2, MCF-7, and SF-268. While (4E,6E)-5-hydroxy-1(4-hydroxy-3-methoxyphenyl)-7-phenylhepta-4,6-dien-3-one
(258) showed weak cytotoxicity against two cancer cell lines of MCF-7 and T98G with $\mathrm{IC}_{50}$ values of 22.68 and $4.44 \mu \mathrm{M}$, respectively. ${ }^{135}$ Meanwhile, 258-267 were proved to be inhibitors of Helicobactor pylori (Hp-Sydney and Hp-F44). ${ }^{129}$ AO-5 (263) showed antiinflammatory activity induced by 12 -O-tetradecanoylphorbol-13acetate (TPA), platelet-activating factor (PAF), and NO. ${ }^{10,136,137}$ Moreover, it exhibited very weak cytotoxic activity against human glioblastoma T98G cells $\left(\mathrm{IC}_{50}=27 \mu \mathrm{M}\right){ }^{138}$ The acetone extract of the rhizomes of $A$. officinarum Hance showed $5 \alpha$-reductase inhibitory effect, which was superior to the drug used in the treatment of androgen-dependent disorders. Therefore, a bioactivity-guided isolation was performed and resulted in the isolation of 263-266 which exerted $5 \alpha$-reductase inhibitory effect with $\mathrm{IC}_{50}$ values ranging from 220 to $390 \mu \mathrm{M}$, indicating potent usage in treating androgen-dependent diseases. ${ }^{139}$ Besides, AO-1 (266) also showed anti-helicobacter pylori, hypolipidemic activities, and NO 
<smiles>COc1cc(OC)c(OC)cc1/C=C/[C@H]1CC[C@H]1/C=C/c1cc(OC)c(OC)cc1OC</smiles>

351 cis-1-(2,4,5-Trimethoxy-E-styryl)-2(2,4,5- trimethoxy-Z-styryl)cyclobutane

352 trans-1-(2,4,5-Trimethoxy-E-styryl)-2(2,4,5-trimethoxy-Z-styryl)cyclobutane

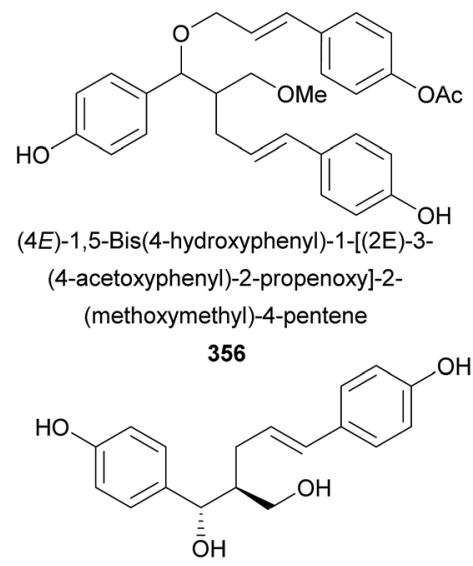

Galanganol B

360<smiles>COc1cc(OC)c(OC)cc1CCC1CCC1CCc1cc(OC)c(OC)cc1OC</smiles>

1,2-Bis(2,4,5-trimethoxy-Z-styryl)-cyclobutane 353<smiles>[R]CC(C/C=C/c1ccc(O)cc1)C(O)c1ccc(O)cc1</smiles>

$357 \mathrm{R}=\mathrm{OMe}$ (4E)-1,5-Bis(4-hydroxyphenyl)2-(methoxymethyl)-4-penten-1-ol $358 \mathrm{R}=\mathrm{OH} \quad(4 E)-1,5-\mathrm{Bis}$ (4-hydroxyphenyl)-2(hydroxymethyl)-4-penten-1-ol<smiles>[R]C(c1ccc(O)cc1)C(C/C=C/c1ccc(O)cc1)COC</smiles>

$354 \mathrm{R}=\mathrm{OMe}$ (4E)-1,5-Bis(4-hydroxyphenyl)-1methoxy-2-(methoxymethyl)-4-pentene $355 \mathrm{R}=\mathrm{OEt} \quad(4 E)-1,5-\mathrm{Bis}$ (4-hydroxyphenyl)-1ethoxy-2-(methoxymethyl)-4-pentene<smiles>Oc1cc(O)c(O)c(CC2OC2c2ccc(O)c(O)c2)c1</smiles>

359<smiles>COC(C/C=C/c1ccc(O)cc1)C(COC(C)=O)c1ccc(O)cc1</smiles>

$361 \mathrm{R}=\mathrm{OMe}$ Conchigeranal $\mathrm{A}$ $362 \mathrm{R}=\mathrm{H} \quad$ Conchigeranal $\mathrm{B}$

Conchigeranal C

363<smiles>COC(c1ccc(O)cc1)C(CO)C/C=C/c1ccc(O)cc1</smiles>

364 Conchigeranal $D$ 365 Conchigeranal E<smiles>O=C/C(=C/c1ccc(O)cc1)C/C=C/c1ccc(O)cc1</smiles><smiles>O</smiles><smiles>[R]C(CO)(C/C=C/c1ccc(O)cc1)[C@@H](O)c1ccc(O)cc1</smiles>

Galanganal

366
$367 \mathrm{R}=\beta-\mathrm{H}$ Galanganol A $368 \mathrm{R}=\alpha-\mathrm{H}$ Galanganol $\mathrm{B}$<smiles>COc1ccc2ccc(=O)oc2c1</smiles>

7-Methoxycoumarin 373<smiles>COC[C@H]1C[C@H](C/C=C/c2ccc(O)cc2)[C@@H](c2ccc(O)cc2)O[C@@H]1c1ccc(O)cc1</smiles>

369 370<smiles>CCOC[C@H]1C[C@H](C/C=C/c2ccc(O)cc2)[C@@H](c2ccc(O)cc2)O[C@@H]1c1ccc(O)cc1</smiles>

372<smiles>COc1cc([C@@H](O)[C@@H](O)Oc2c(OC)cc(/C=C/CO)cc2OC)ccc1OC(F)(Cl)Cl</smiles>

374<smiles>COC[C@H]1C[C@H](C/C=C/c2ccc(O)cc2)[C@@H](c2ccc(O)cc2)O[C@@H]1c1ccc(O)cc1</smiles><smiles>COc1cc([C@@H]2Oc3c(O)cc(CCCO)cc3[C@@H]2CO)ccc1OC(C)=O</smiles>

2,3-Dihydro-2-(4- $\beta$-D-glucopyranosyl3-methoxyphenyl)-3-hydroxymethyl-7-

hydroxy-5-benzofranpropanol

375

Fig. 6 Lignans from Alpinia species.

production inhibitory activity. ${ }^{\mathbf{1 1 0 , 1 4 0 , 1 4 1}}$ AO-2 (267) was identified as an inhibitor of prostaglandin (PG) biosynthesis and exerted antioxidant activity. ${ }^{\mathbf{1 4 2 , 1 4 3}}$ It is interesting to note that dihydroyashabushiketol (264), AO-1 (266), and AO-2 (267) were firstly reported as planar structures, and later, their absolute configurations were established as 264a, 266a, and 267a, respectively. ${ }^{\mathbf{1 3 6 , 1 4 4}}$

7-(4"-Hydroxy-3"-methoxyphenyl)-1-phenyl-3,5-heptadione (268) also exhibited prostaglandin biosynthesis inhibitory effect 
<smiles>[R]c1ccc(-c2oc3cc([R])cc(O)c3c(=O)c2[R])cc1</smiles>

$$
\begin{array}{llllll} 
& \mathrm{R}_{1} & \mathrm{R}_{2} & \mathrm{R}_{3} & \\
\cline { 1 - 3 } 376 & \mathrm{OMe} & \mathrm{H} & \mathrm{H} & \text { Tectochrysin } \\
\mathbf{3 7 7} & \mathrm{OH} & \mathrm{H} & \mathrm{H} & \text { Chrysin } \\
\mathbf{3 7 8} & \mathrm{OH} & \mathrm{H} & \mathrm{OH} & \text { Apigenin } \\
\mathbf{3 8 3} & \mathrm{OH} & \mathrm{OMe} & \mathrm{OMe} & \text { Kaempferol-3,4'-dimethylether }
\end{array}
$$$$
384 \mathrm{OH} \quad \mathrm{OH} \quad \mathrm{H} \quad \text { Galangin }
$$$$
385 \mathrm{OH} \mathrm{OH} \text { OMe Kaempferide }
$$$$
386 \mathrm{OH} \quad \mathrm{OH} \quad \mathrm{OH} \text { Kaempferol }
$$$$
387 \mathrm{OH} \quad \mathrm{OMe} \quad \mathrm{OH} \text { 3-Methoxykaempferol }
$$$$
388 \mathrm{OMe} \mathrm{OH} \text { OMe 3,5-Dihydroxy-7,4'-dimethoxyflavone }
$$$$
389 \mathrm{OMe} \mathrm{OH} \quad \mathrm{H} \quad \text { Izalpinin }
$$$$
390 \mathrm{OH} \quad \mathrm{OMe} \quad \mathrm{H} \quad \text { 3-Methylethergalangin }
$$$$
391 \mathrm{OMe} \mathrm{OMe} \mathrm{OH} \text { Kumatakenin }
$$$$
392 \mathrm{OMe} \mathrm{OH} \quad \mathrm{OH} \text { Rhamnocitrin }
$$$$
396 \mathrm{OMe} \mathrm{OMe} \text { OMe 5-Hydroxy-3,7,4' -trimethoxyflavone }
$$<smiles>[R]c1ccc([C@H]2CC(=O)c3c([R1])cc(O)cc3O2)cc1</smiles><smiles>[R]C[R]</smiles>

$397 \mathrm{OH} \quad \mathrm{H}$ Pinocembrin $398 \mathrm{OMe} \mathrm{H}$ Alpinetin $399 \mathrm{OMe} \mathrm{OH} \mathrm{7,4'-Dihydroxy-5-}$ methoxy flavanone<smiles>CC(=O)OC1C(=O)c2c(O)cc(O)cc2O[C@H]1c1ccccc1</smiles>

3-O-Acetylpinobanksin 403<smiles>[R]c1ccc(/C=C/C(=O)c2c([R])cc([R])c([R3])c2[R])cc1</smiles>

$$
\begin{array}{lllll}
R_{1} & R_{2} & R_{3} & R_{4} & R_{5} \\
\hline
\end{array}
$$

$410 \mathrm{OH} \quad \mathrm{OMe} \mathrm{H}$ OMe $\mathrm{H}$ Flavokawin $\mathrm{B}$

$411 \mathrm{OH} \quad \mathrm{OH} \quad \mathrm{H}$ OMe $\mathrm{H}$ Cardamomin

$412 \mathrm{OH} \quad \mathrm{OH} \quad \mathrm{OH}$ OH $\mathrm{H} \quad 2^{\prime}, 3^{\prime}, 4^{\prime}, 6^{\prime}-$ Tetrahydroxychalcone

413 OMe OMe H OMe H 2',4',6'-Trimethoxychalcone

$414 \mathrm{OH} \quad \mathrm{OMe} \mathrm{H} \quad \mathrm{OH} H$ Pinostrobin chalcone

$415 \mathrm{OH} \mathrm{OH} \quad \mathrm{H} \quad \mathrm{OMe} \mathrm{OH}$ Helichrysetin

$416 \mathrm{OMe} \mathrm{OH} \quad \mathrm{H}$ OMe OH 2,6-Dimethoxy-4,4-dihydroxychalcone

$417 \quad \mathrm{H} \quad \mathrm{OH} \quad \mathrm{H} \quad \mathrm{H} \quad \mathrm{OH}$ 4,4-Dihydroxychalcone

$418 \mathrm{OH} \quad \mathrm{OH} \quad \mathrm{OH} \quad \mathrm{OH}$ Pinocembrin chalcone<smiles>[R9]c1ccc([R8])c(-c2cc(=O)c3c(O)cc(OC)c(OC)c3o2)c1</smiles>

\begin{tabular}{cccc} 
& $\mathrm{R}_{1}$ & $\mathrm{R}_{2}$ & \\
\hline 379 & $\mathrm{H}$ & $\mathrm{H}$ & 5-Hydroxy-7,8-dimethoxyflavone
\end{tabular}

380 OMe H 5-Hydroxy-2',7,8-trimethoxyflavone

381 OMe OMe 5-Hydroxy-7,8,2',5'-tetramethoxyflavone<smiles>COc1ccc(-c2cc(=O)c3c(OC)cc(O)cc3o2)cc1OC</smiles>

5-Hydroxy-3',4',7trimethoxy flavanone 382<smiles>[R]c1cc(O)c2c(=O)c(O)c(-c3ccc([R])c(O)c3)oc2c1</smiles>
\begin{tabular}{lll}
$\mathrm{R}_{1}$ & $\mathrm{R}_{2}$ \\
\hline $393 \mathrm{OH}$ & $\mathrm{OH}$ & \\
Quercetin
\end{tabular} 394 OMe OMe Ombuine

4',5,7-Trimethoxyflavonol

395<smiles>O=C1c2c(O)cc(O)cc2O[C@H](c2ccccc2)[C@@H]1O</smiles>

401<smiles>O=C(/C=C/c1ccccc1)O[C@H]1C(=O)c2c(O)cc(O)cc2O[C@H]1c1ccccc1</smiles>

$(2 R, 3 S)$-Pinobaksin-3-cinnamate

402<smiles></smiles>

-Hydroxy-7,4'-dimethoxy-2" S(2,4,5-trimethoxy-E-styryl)-tetrahdrofuro[4" $R, 5 " R: 2,3$ ]flavanonol

404<smiles>O=C1c2c(O)cc(O)cc2O[C@H](c2ccc(O)cc2)[C@@H]1O</smiles>

406

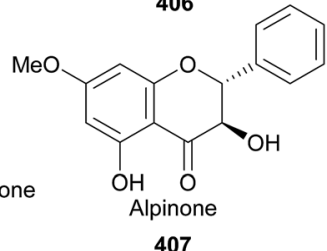

407<smiles>COc1ccc([C@]23Oc4cc(OC)cc(O)c4C(=O)[C@@]2(O)O[C@H](/C=C/c2ccccc2)[C@H]3O)cc1</smiles>

(2,4,5-trimethoxy-Estyryl)tetrahydrofuro[4" $R, 5 " R: 2,3]$ flavanonol

405<smiles>[R]c1cc(O)c(C(=O)CCc2ccccc2)c(OC)c1</smiles>

$408 \mathrm{R}=\mathrm{OH}$ Uvangoletin

$409 \mathrm{R}=\mathrm{OMe}$ Dihydroflavokawin $\mathrm{B}$

Fig. 7 Flavonoids from Alpinia species.

with $\mathrm{IC}_{50}$ values of $50 \mu \mathrm{M} .{ }^{143} \mathrm{AO}-4$ (269) was found to have marked inhibitory effect on TPA-induced inflammation and antioxidant activity. ${ }^{142,144}$ 6-Hydroxy-1,7-diphenyl-4-en-3-heptanone (270) was a PAF inhibitor. ${ }^{137} \mathrm{AO}-3$ (271) and (5S)-5-methoxy-1,7-diphenyl-3heptanone (272) displayed potent inhibitory effects on TPAinduced inflammation in mice with $50 \%$ of inhibition at a dose 
<smiles>[R2]CCCCCC/C=C/C(c1ccc(O)cc1)c1c(O)cc(OC)c(C(=O)/C=C/c2ccc([R7])cc2)c1O</smiles><smiles>Oc1cc(O)c2c(c1)O[C@H](c1ccc(O)c(O)c1)[C@H](O)C2</smiles>

$(+)$-Catechin

423<smiles>Oc1cc(O)c2c(c1)O[C@H](c1ccc(O)c(O)c1)[C@H](O)C2</smiles>

(+)-Epicatechin

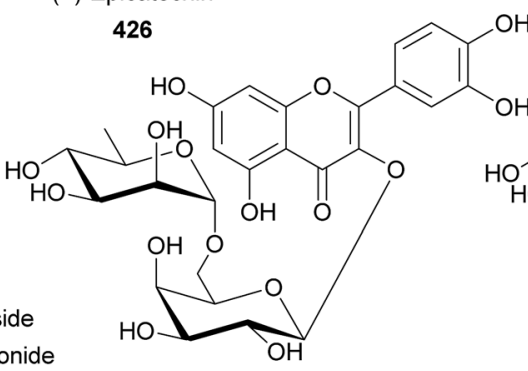

Quercetin 3-O- $\beta$-D-rhamnosyl$(1,6)$-galactoside 432

$\mathrm{HO}$<smiles>COc1c(-c2ccc(C)cc2)oc2cccc(O)c2c1=O</smiles>

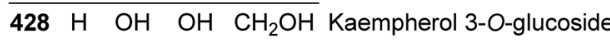
$429 \mathrm{H} \mathrm{OH} \mathrm{OH} \mathrm{COOH}$ Kaempherol 3-O-glucuronide $430 \mathrm{OH} \mathrm{OH} \quad \mathrm{OH} \quad \mathrm{CH}_{2} \mathrm{OH}$ Quercetin 3-O-glucoside $431 \mathrm{OH} \mathrm{OH} \mathrm{OH} \mathrm{COOH}$ Quercetin 3-O-glucuronide<smiles>[R]c1cc([C@H]2Oc3cc(O)cc(O)c3C[C@H]2O)cc(O)c1O</smiles>

$424 \mathrm{R}=\mathrm{H} \quad$ Epicatechin $425 \mathrm{R}=\mathrm{OH}$ Galloepicatechin<smiles>[R]c1ccc(-c2oc3cc(O)cc(O)c3c(=O)c2OC([O-])([O-])O[Na])cc1[R]</smiles>

Kaempferide-3-O- $\beta$-D-glucoside

427

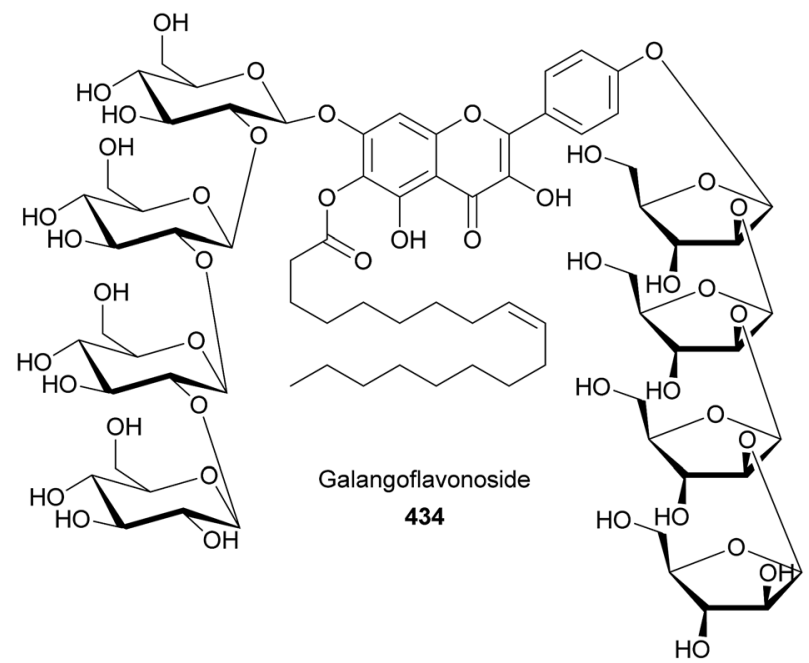

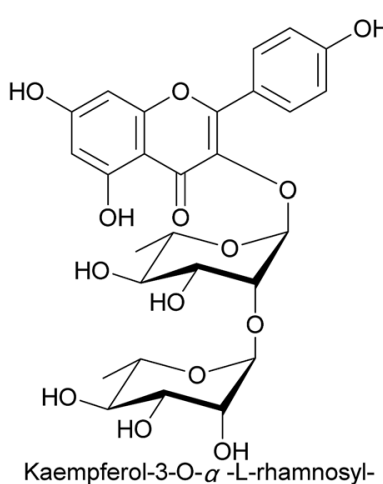
$(1 \rightarrow 2)$-O-L-rhamnoside 435<smiles>O=c1c(O)c(-c2ccc(O)cc2O)oc2cc(OC3CCCCC3)cc(O)c12</smiles>

Morin-7-O- $\beta$-D-glucopyranoside 437<smiles>[R]Oc1c(C(C)=O)oc2cc(O)cc(O)c2c1=O</smiles>

$\mathrm{R}=\mathrm{Me}$

438 Quercetin 3-O-(2, 6-di-Orhamnopyranosylgalactopyranoside) 439 Isorhamnetin 3-O-(2, 6-di-Orhamnopyranosylgalactopyranoside)

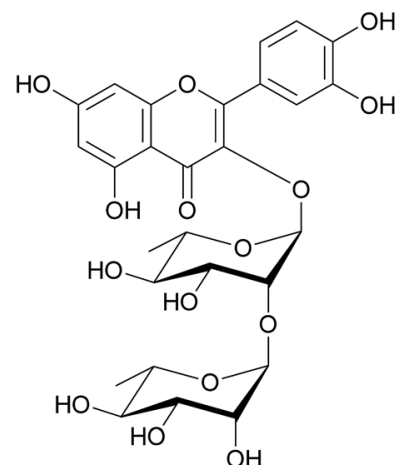

Quercetin-3-O- $\alpha$-L-rhamnosyl-

$(1 \rightarrow 2)-O-\alpha$-L-rhamnoside 436

Fig. 7 Flavonoids from Alpinia species (continued).

of 0.8-2.7 $\mu \mathrm{mol}$ per ear. ${ }^{144}$ (3R,5R)-1-(4-Hydroxyphenyl)-7-phenyl-3,5heptanediol (273) showed significantly antiemetic effect induced by $\mathrm{CuSO}_{4}$ with $37.7 \%$ inhibition at a dose of $50 \mathrm{mg} \mathrm{kg}{ }^{-1} \cdot{ }^{27,145}$
Investigation on seeds of A. blepharocalyx K. Schum. led to the isolation of ten cyclic diarylheptanoids (274-283). ${ }^{\mathbf{1 1 5}, \mathbf{1 4 6 - 1 4 8}}$ Rhizomes of $A$. officinarum Hance provided 3,6-furan-7-(4"- 


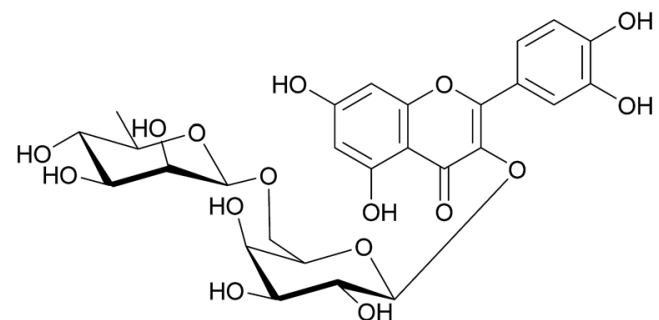

Isorhamnetin-3-O- $\beta$-D-galactosyl-

$(6 \rightarrow 1)-\alpha$-L-rhamnoside 441<smiles>[R2]c1cc(-c2oc3cc(O)cc(O)c3c(=O)c2OCC)ccc1O</smiles>

$\begin{array}{ll}\mathrm{R}_{1} & \mathrm{R}_{2}\end{array}$

442 Rutinoside $\mathrm{OH}$ Rutin

443 Rutinoside H Kaempferol-3-O-rutinoside<smiles>[Y]C(CCc1cc(O)ccc1C(=O)CCc1ccc(O)cc1OC)(c1ccc(O)cc1)c1cc(CCC(=O)c2ccc(O)cc2)c(OC)cc1OC</smiles>

445a $y^{\prime}-S \quad y^{\prime \prime}-S$

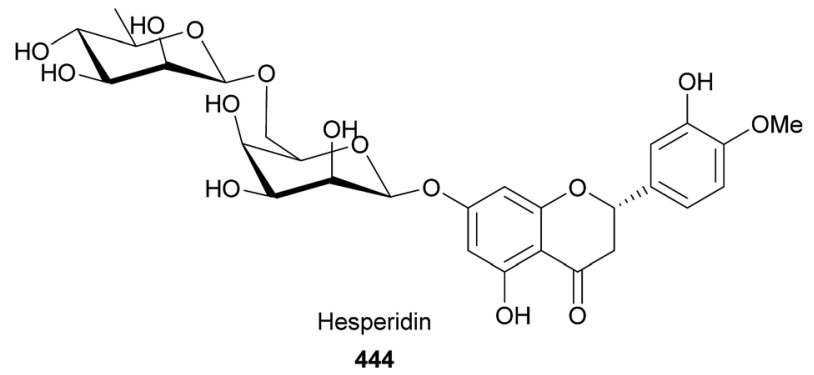<smiles>COc1cc(O)c(C(C)(CCc2ccc(O)cc2OC)c2ccc(O)cc2)cc1CCC(=O)c1ccc(O)cc1</smiles>

446a $\vee-R$

446b $\gamma-S$

Fig. 7 Flavonoids from Alpinia species (continued).<smiles>Oc1ccc(/C=C\C/C=C\c2ccc(O)cc2)cc1</smiles>

[Di-(p-hydroxy-cis-styryl)] methane

447<smiles>CCCCOC(=O)c1ccccc1C(=O)OCCCC</smiles>

Dibutyl phthalate

451<smiles>COc1cc(/C=C/COC(C)=O)ccc1OC(C)=O</smiles>

trans-Coniferyl diacetate

468<smiles>CC(=O)Oc1ccc(/C=C/COC/C=C/c2ccc(OC(C)=O)cc2)cc1</smiles>

4,4'[(2E,2'E)-Bis(prop-2-ene)-1,1'-oxy]-diphenyl-7,7'-diacetata 469<smiles>CCCCCCCC(=O)/C=C/c1ccc(O)cc1</smiles>

$\mathrm{R}_{1} \quad \mathrm{R}_{2}$

$452 \mathrm{OH} \quad \mathrm{CH}_{2} \mathrm{OH}$ (E)-p-Coumaryl alcohol $453 \mathrm{OH} \quad \mathrm{CH}_{2} \mathrm{OMe}(E)$ - $p$-Coumary/ alcohol $\gamma$ O-methy/ ether

$454 \mathrm{OH} \quad \mathrm{CHO}$ trans-p-Hydroxycinnamaldehyde

$455 \mathrm{OH} \quad \mathrm{CH}_{2} \mathrm{OAc}$ trans-p-Hydroxycinnamyl acetate

$456 \mathrm{OH} \quad \mathrm{CH}_{2} \mathrm{OH}$ trans-p-Coumaryl alcohol

$457 \mathrm{OAc} \quad \mathrm{CH}_{2} \mathrm{OAc}$ trans-p-Coumary/ diacetate

$458 \mathrm{OAc} \mathrm{CH}_{2} \mathrm{OH}$ trans-p-Acetoxycinnamyl alcohol

$459 \mathrm{OAc} \mathrm{CHO}$ trans-p-Hydroxy/cinnamaldehyde acetate $460 \mathrm{OH} \quad \mathrm{COOH} \quad p$-Coumaric acid

$461 \mathrm{H} \quad$ CoOMe Methyl trans-cinnamate

$462 \mathrm{OH}$ COOMe Methyl $p$-hydroxycinnamate

$463 \mathrm{OH}$ COMe Methyl $p$-hydroxycinnamyl ketone<smiles>C=C[C@H](OCC)c1ccc(OC(C)=O)cc1</smiles>

(S)-1'-Ethoxy chavicol acetate 470

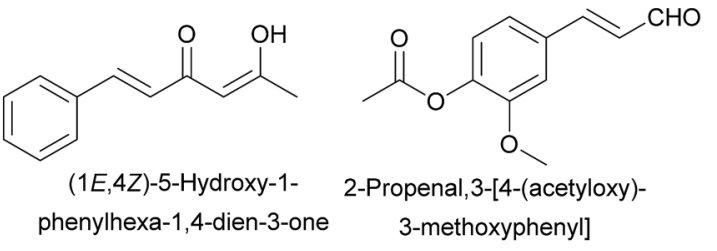

449<smiles>[R]c1ccc(C([R3])C=C)cc1[R]</smiles>

\begin{tabular}{lll}
$\mathrm{R}_{1}$ & $\mathrm{R}_{2}$ & $\mathrm{R}_{3}$ \\
\hline
\end{tabular}

$464 \mathrm{H} \quad$ OAc OAc 1'S-1'-Acetoxychavicol acetate 465 OMe OAc OAc 1'-Acetoxyeugenol acetate

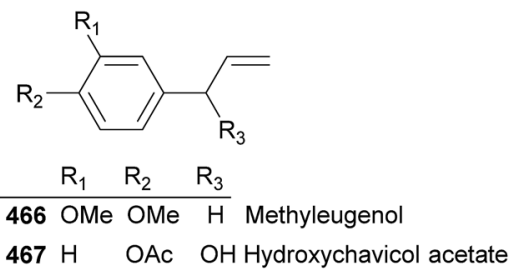<smiles>[R]c1ccc(/C=C/COCC)cc1</smiles>

$471 \mathrm{R}=\mathrm{OCOMe}(E)-4-$ Acetoxy cinnamyl ethyl ether $472 \mathrm{R}=\mathrm{OH} \quad(E)-p$-Coumaryl alcohol ethyl ether

Fig. 8 Phenolics from Alpinia species. 
<smiles>COc1ccc(/C=C/CO)cc1OC</smiles>

trans-3,4-Dimethoxycinnamyl alcohol

473<smiles>COc1cc(OC)c(OC)cc1/C=C/C=O</smiles>

(E)-2,4,5-Trimethoxycinnamal dehyde

478<smiles>COc1cc(OC)c(C(=O)O)cc1OC</smiles>

2,4,5-Trimethoxybenzoic acid 482<smiles>O=C(O)c1cc(O)ccc1O</smiles>

2,5-Dihydroxybenzoic acid 487<smiles>COc1cc(C(=O)O)ccc1O</smiles>

Vanillic acid 488<smiles>COc1cc(OC)c(OC)cc1/C=C/C(=O)O</smiles>

2,4,5-Trimethoxycinnamic acid

483<smiles>[R]c1ccc(C=O)cc1</smiles>

$490 \mathrm{R}=\mathrm{OH}$ 4-Hydroxybenzaldehyde $492 \mathrm{R}=\mathrm{H} \quad$ Benzaldehyde

474<smiles>COc1cc(OC)c(OC)cc1C=O</smiles>

2,4,5-Trimethoxybenzaldehyde 479

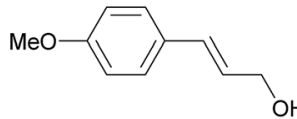

(E)-p-Acetoxycinnamyl alcohol trans-4-Methoxycinnamyl alcohol<smiles>COc1cc(-c2ccccc2O)oc(=O)c1</smiles>
6-(2-Hydroxy-phenyl)4-methoxy-2-pyrone

496

4-Hydroxy-2-(2,4,5-trimethoxyphenyl)-2-E-butenal

480<smiles>COc1c(O)cc(C(=O)O)cc1O</smiles>

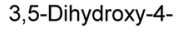

methoxybenzoic acid

484

Isovanillin

491

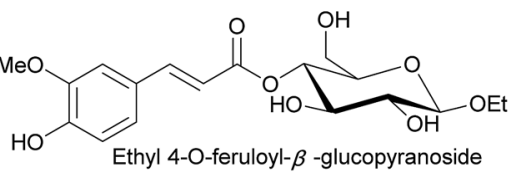

497

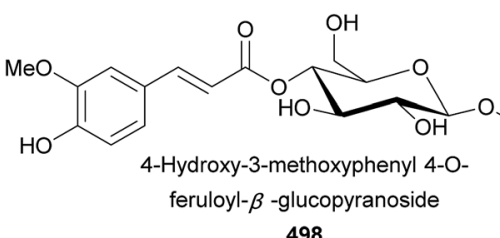<smiles>COc1ccccc1O</smiles>

498<smiles>C=CCc1ccc(OC(=O)OCc2ccccc2)c(O)c1</smiles>

1-O- $\beta$-D-Glucopyranosyl2-hydroxy-4-allylbenzene 502

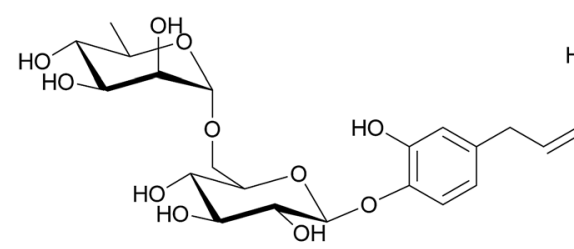

1-O-(6-O- $\alpha$-L-Rhamnopyranosyl$\beta$-D-glucopyranosyl)-2-hydroxy4-allylbenzene

503
1-O- $\beta$-D-Glucopyranosyl-4-allylbenzene

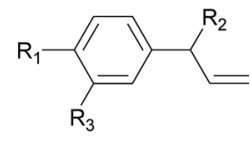

\begin{tabular}{lll}
$R_{1}$ & $R_{2}$ & $R_{3}$ \\
\hline
\end{tabular}

$476 \mathrm{OACH}$ H Chavicol acetate 477 OAC OAC OAC 1'S-1'-Acetoxyeugenol acetate<smiles>COc1cc(/C=C/C(=O)O)ccc1O</smiles>

481<smiles>COc1ccc(C=O)cc1O</smiles>

Benzyl $\beta$-D-glucopyranoside

499<smiles>C=CCc1ccc(O)c(OC(Cl)OCl)c1</smiles>

1-Hydroxy-2-O- $\beta$-D-

glucopyranosyl-4-allylbenzene

501

Fig. 8 Phenolics from Alpinia species (continued).

hydroxy-3" ${ }^{\prime \prime}$-methoxyphenyl)-1-phenylheptane (284). ${ }^{131}$ From the seeds of $A$. katsumadai, 285-292 were obtained, ${ }^{\mathbf{1 4 9}}$ three of which (285-287) displayed weak antiproliferative activities against four cancer cell lines of NCI-H460, HeLa, SMMC-7721, and HCT-116 with $\mathrm{IC}_{50}$ values of 15.39-42.24 mM. ${ }^{115,149}$ A. blepharocalyx K. Schum. was the source of 293-305. ${ }^{115,148,150,151}$ However, 


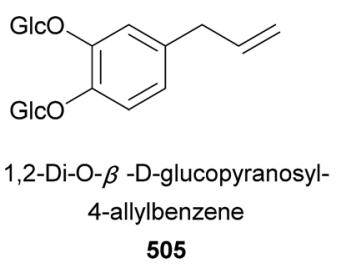

505
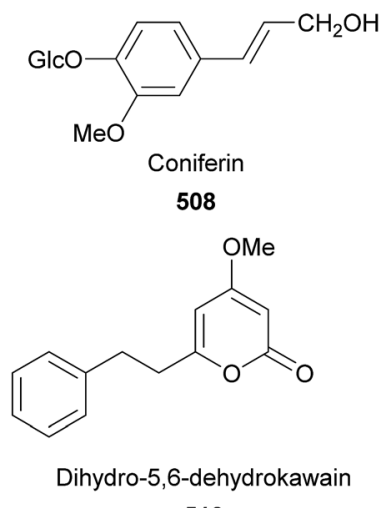

510

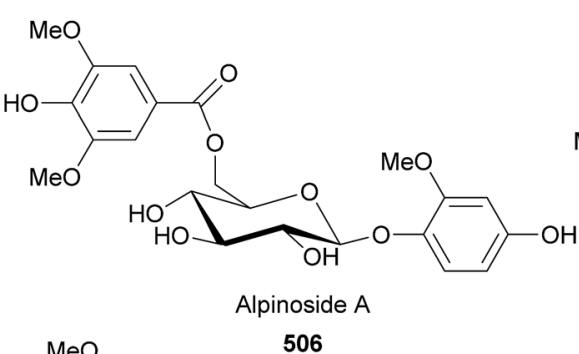

506
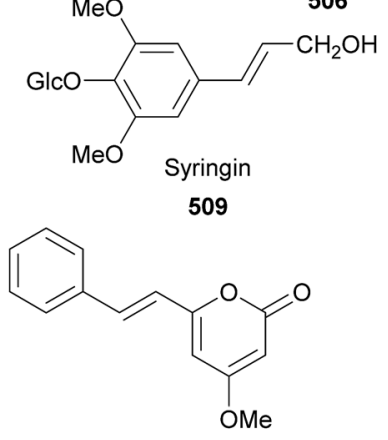

5,6-Dehydrokawain

511

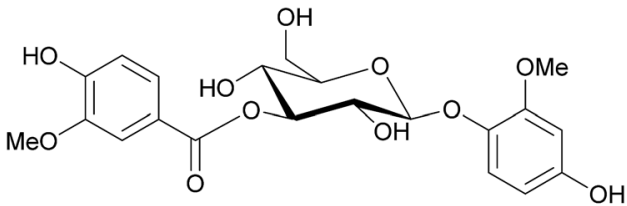

4-Hydroxy-2-methoxyphenol- $\beta$ -

D-\{3"-O-[4'-hydroxy-3'-methoxy

(benzoate)]\}-glucopyranoside

507

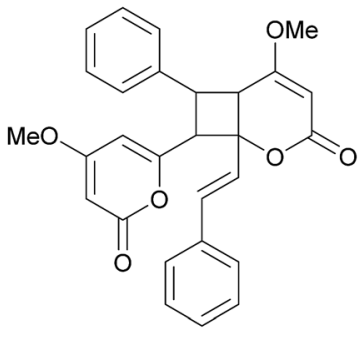

AS-II

511a

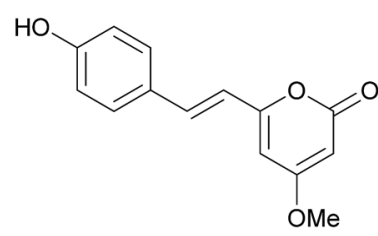

4-Hydroxy-5,6-dehydrokawain

512

Fig. 8 Phenolics from Alpinia species (continued).

the stereochemistry at C- $9^{\prime \prime}$ of six stereoisomerics (294/295, 296/ 297, 298/299, 300/301, 302/303, 304/305) remained unsolved. Calyxin J (298), epicalyxin J (299), calyxin K (300), and epicalyxin $\mathrm{K}$ (301) showed marked anti-proliferative activity against human HT-1080 fibrosarcoma cells with $\mathrm{ED}_{50}$ values from $0.3-$ 8.2 $\mu \mathrm{M} .{ }^{115,152}$ Compounds 302-305 were proved to inhibit NO production in endotoxin activated murine macrophage J774.1 with $90-94 \%$ inhibitory rate at a concentration of $100 \mu \mathrm{g}$ $\mathrm{mL}^{-1} \cdot{ }^{151}$ Seeds of A. katsumadai Hayata provided 306-318. Calyxins Q (306) and R (307) exerted potent antiproliferative activities against four cancer cell lines of NCI-H460, HeLa, SMMC-7721, and HCT-116 at the level of $\mathrm{IC}_{50}$ values of 15.3-42.2 $\mu \mathrm{M} .{ }^{\mathbf{1 4 9}}$ Calyxin B (319) and epicalyxin B (320) were obtained from A. blepharocalyx K. Schum. and A. pinnanensis as NO production inhibitiors. ${ }^{\mathbf{1 1 5 , 1 5 1}}$ In addition, 319 showed potent antiproliferative activity against human HT-1080 fibrosarcoma cells with an $\mathrm{ED}_{50}$ value of $0.69 \mu \mathrm{M} .{ }^{\mathbf{1 4 8}}$ Both $A$. pinnanensis $\mathrm{T}$. L. Wu et Senjen and A. katsumadai Hayata yielded alpinnanin B (321). ${ }^{118,124}$ From A. katsumadai Hayata and A. blepharocalyx K. Schum., epicalyxin $\mathrm{H}$ (322) and calyxin $\mathrm{H}$ (323) were isolated. 118,124,153 Epicalyxin $\mathrm{H}$ was identified as NO production inhibitor. ${ }^{115,153}$ Seeds of A. blepharocalyx yielded 324330. ${ }^{115,152,154}$ It's worth mentioning that all three structures of calyxin L (325), epicalyxin F (327), and calyxins F (328) in the Scifinder were wrong. Out of a serious of diarylheptanoids bearing a chalcone or a flavanone moiety, epicalyxins I (326), F (327), and calyxin F (328) were shown to possess strong antiproliferative activities toward colon 26-L5 carcinoma and HT1080 fibrosarcoma with $\mathrm{IC}_{50}$ values ranging from 0.5 to 10.1 $\mu \mathrm{M} .{ }^{\mathbf{1 1 5 , 1 5 0}}$ Meanwhile, $\mathbf{3 2 6}$ and $\mathbf{3 2 7}$ were cytotoxic against human fibrosarcoma cells with $\mathrm{IC}_{50}$ values ranging from 0.9 to 12.1 $\mu \mathrm{M} .{ }^{152}$ 6-Hydroxycalyxin $\mathrm{F}$ (329) and calyxin A (330) demonstrated NO production inhibitory activities with $\mathrm{IC}_{50}$ values of
49 and $62 \mu \mathrm{M}$, respectively. ${ }^{115,150}$ Rhizomes of $A$. pinnanensis $\mathrm{T}$. L. $\mathrm{Wu}$ et Senjen provided deoxycalyxin A (331), alpinnanins A (332), and $\mathrm{C}(333) .{ }^{118}$ In addition, 331 was also found in A. blepharocalyx K. Schum. ${ }^{115}$ While officinin A (334) was obtained from rhizomes of $A$. officinarum Hance. ${ }^{155}$

Five dimeric diarylheptanoids (335-339) were obtained from rhizomes of $A$. officinarum Hance. ${ }^{135,136,138,156,157}$ Only alpinin C (338) displayed selective cytotoxic against MCF-7 $\left(\mathrm{IC}_{50}=62.3\right.$ $\mu \mathrm{M})$ and T98G cells $\left(\mathrm{IC}_{50}=57.3 \mu \mathrm{M}\right) \cdot{ }^{135}$ Seeds of $A$. blepharocalyx K. Schum. provided 340-344 possessing two diarylheptanoid units and a chalcone moiety. ${ }^{\mathbf{1 1 5 , 1 4 6 , 1 5 3}}$ Both blepharocalyxins A (340) and B (341) showed concentration-dependent inhibition in the range of $1-100 \mu \mathrm{g} \mathrm{mL} \mathrm{m}^{-1}$ against NO production in endotoxin-activated murine macrophages J774.1. ${ }^{158}$ Blepharocalyxins C-E (342-344) were tested for antiproliferative activities against two tested cancer cells, blepharocalyxin D (343) exhibited the strongest effect against highly liver-metastatic murine colon 26-L5 carcinoma cells $\left(\mathrm{ED}_{50}=3.6 \mu \mathrm{M}\right)$, whereas blepharocalyxin E (344) showed the strongest activity against human HT-1080 fibrosarcoma cells $\left(\mathrm{ED}_{50}=9.02 \mu \mathrm{M}\right) .{ }^{\mathbf{1 1 5 , 1 4 6 , 1 5 9}} \mathrm{It}$ is worth mentioning that the stereochemistry at C-I-5 position for 343 in Scifinder was $S$, which was not correct and should be revised as $R$. Moreover, the two diarylheptanoid moieties in 344 were wrongly connected through C-I- 6 and C-II- 5 by Scifinder. Instead, it should be joined through C-I-6 and C-II-7. Two unusual diarylheptanoid derivatives, neocalyxin A (345) and its epimer neocalyxin B (346), were found from the seeds of $A$. blepharocalyx K. Schum., with the stereochemistry at C- $9^{\prime \prime}$ undetermined. ${ }^{\mathbf{1 1 5 , 1 5 2}}$

Rhizomes of A. officinarum Hance produced officinaruminane B (347), a diarylheptanoid coupled with a monoterpene unit. ${ }^{131}$ Investigation on seeds of A. katsumadai Hayata identified two novel anti-emetic diaryllheptanoids, katsumadains A (348) and B 


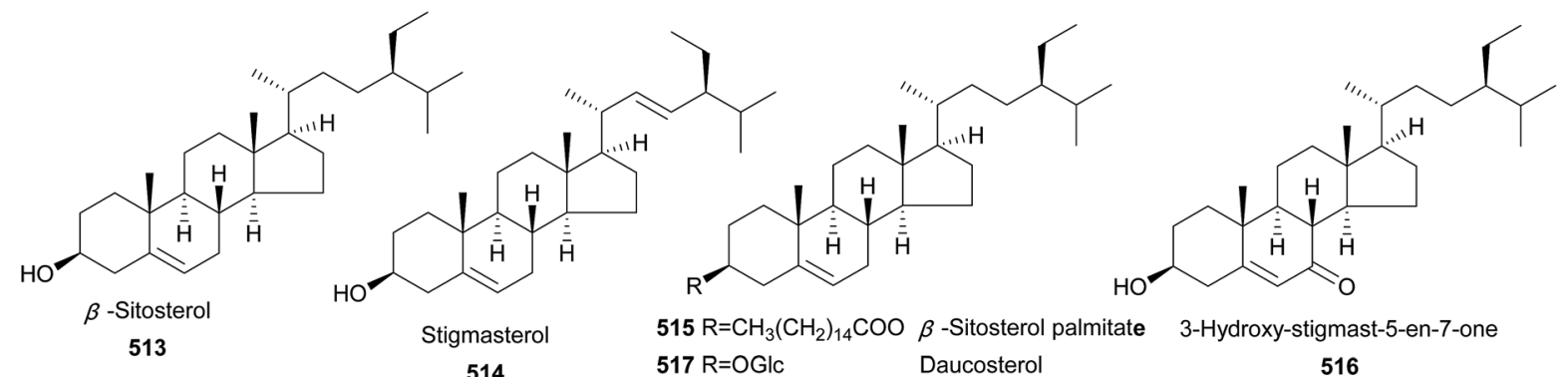

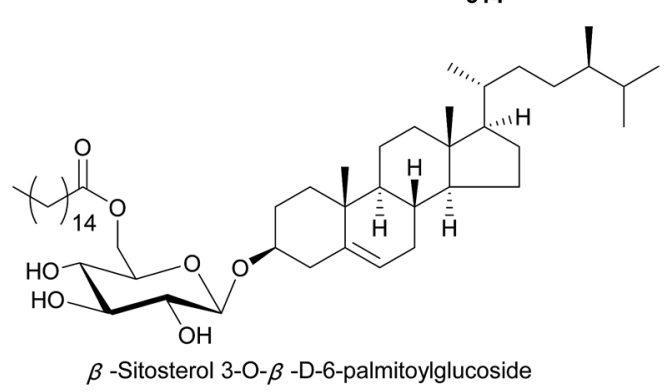

518 $517 \mathrm{R}=\mathrm{OGlC}$

Daucosterol

516

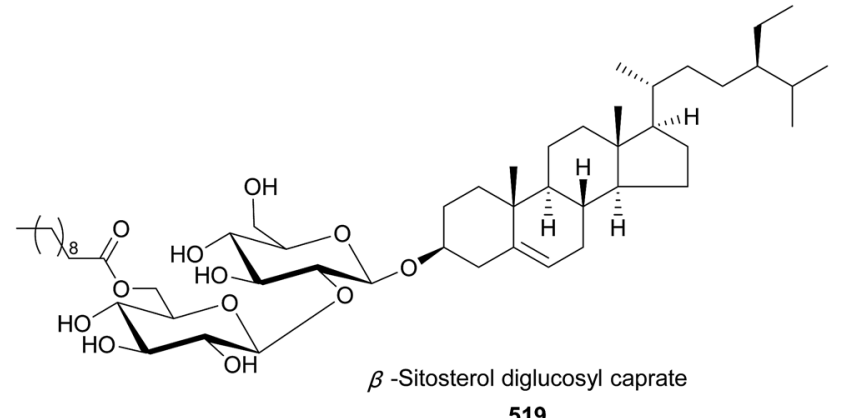<smiles>O=C(CCc1ccccc1)c1cc(C(=O)CCc2ccccc2)c(CCc2ccccc2)nc1CCc1ccccc1</smiles>

520

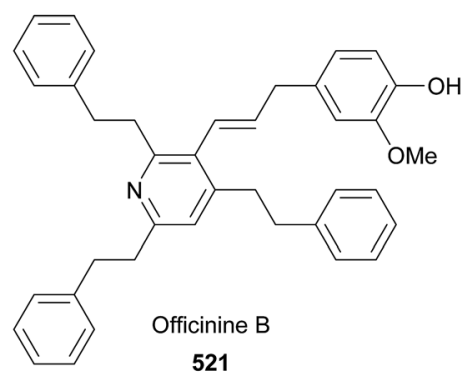

521<smiles>CC(=O)OC[C@H](Cc1ccccc1)NC(=O)[C@H](Cc1ccccc1)NC(=O)c1ccccc1</smiles><smiles>[R]Oc1cc(/C=C/c2ccc([R9])cc2)cc(O[R2])c1</smiles>

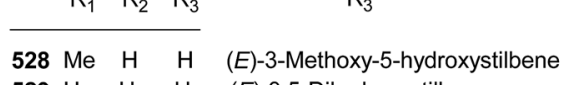

$529 \mathrm{H} \quad \mathrm{H} \quad \mathrm{H}$ (E)-3,5-Dihydroxystilbene

$530 \mathrm{Me} \mathrm{Me} \mathrm{H}$ (E)-3,5-Dimethoxystilbene

$531 \mathrm{H} \quad \mathrm{H}$ OMe (E)-3,5-Dihydroxy-4'-methoxystilbene<smiles>[R]c1cc(O)cc(C=Cc2ccccc2)c1</smiles>

$532 \mathrm{R}=\mathrm{OMe}(\mathrm{Z})-3-$ Methoxy-5-hydroxystilbene $533 \mathrm{R}=\mathrm{OH} \quad(Z)-3,5-$ Dihydroxystilbene<smiles>Nc1ncnc2c1ncn2[C@@H]1O[C@H](CO)[C@@H](O)[C@H]1O</smiles>

523<smiles></smiles><smiles>Oc1ncnc2[nH]cnc12</smiles><smiles>Nc1ncnc2nc[nH]c12</smiles>

522<smiles>CC1([13C](=O)C(=O)OCC(O)CO)CC1</smiles><smiles>CC(C)(C)C(=O)O</smiles><smiles>O=C(O)CCC(=O)O</smiles><smiles>O=C(O)c1cccnc1</smiles>

Nicotinic acid

527 
Number of publications

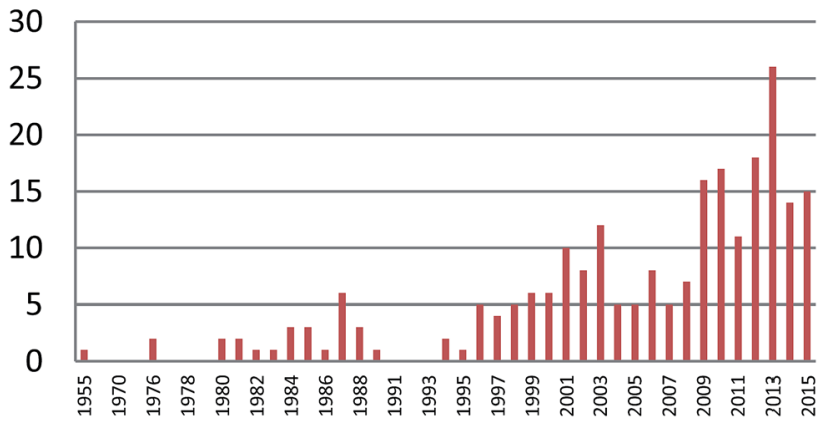

Fig. 10 The number of publications on Alpinia since 1955.

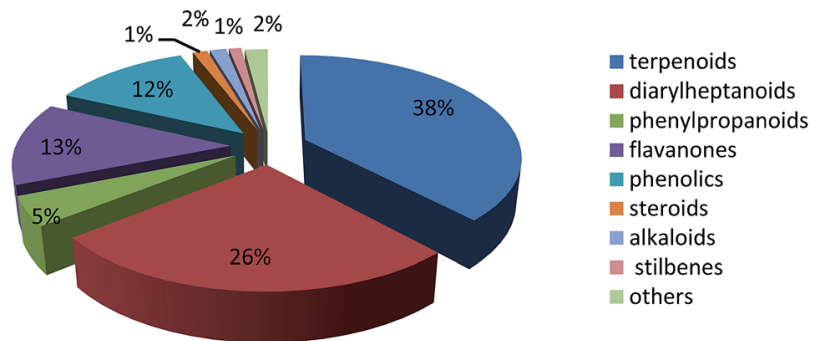

Fig. 11 The percentage of each type of compounds from Alpinia species.

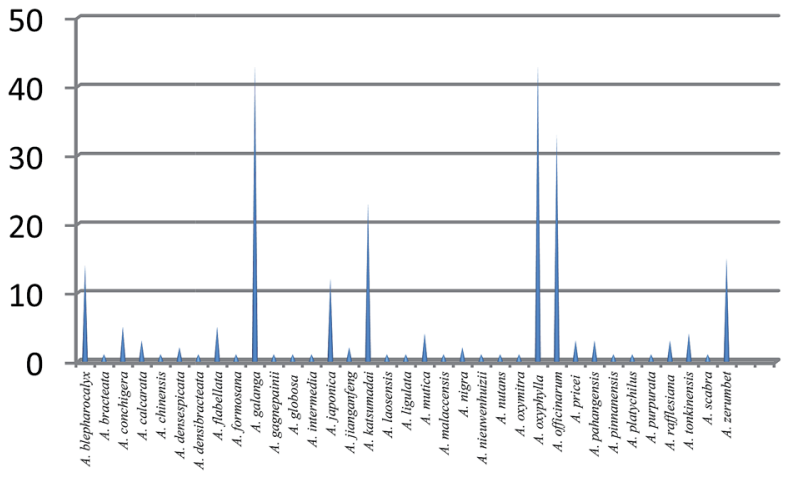

Fig. 12 The number of published papers for each investigated Alpinia species on chemical constituents and their bioactivities over last six decades since 1955

\section{Lignans}

Twenty-four lignans (351-374) were reported from the genus of Alpinia (Fig. 6). Separation for leaves of A. flabellata Ridley resulted in the isolation of 351-353, three phenylbutanoid dimers bearing a novel tetracyclic moiety. ${ }^{161,162}$ cis-1-(2,4,5-Trimethoxy- $E$-styryl)-2-(2,4,5-trimethoxy- $Z$-styryl)cyclobutane (351) and trans-1-(2,4,5-trimethoxy- $E$-styryl)-2-(2,4,5-trimethoxy- $Z$ styryl)cyclobutane (352) showed weak antibacterial against Staphylococcus aureus with MIC values of 5.0 and $2.5 \mathrm{mM}$, respectively. ${ }^{161}$ Furthermore, 351 significantly decreased the ovalbumin permeability in intestinal cells. ${ }^{\mathbf{1 6 1}}$
Rhizomes of A. officinarum Hance yielded 354-358 containing a rare $\beta-\gamma$ linkage. All five compounds exhibited weak antioxidant activities against the autoxidation of methyl linoleate in bulk phase. ${ }^{163}$ Extracts of seeds of A. katsumadai Hayata afforded antiemetic katsumadin (359) with antiemetic activity on $\mathrm{CuSO}_{4}$-induced emesis in young quail. ${ }^{\mathbf{1 2 1}}$ Galanganol B (360) was isolated from rhizomes of A. galanga (L.) Willd. ${ }^{164}$ Investigation on the whole plant of $A$. conchigera afforded eight rare 8$9^{\prime}$ linked neolignans 361-368. ${ }^{165}$ Although conchigeranals D (364) and E (365) shared the same planar structure, their relative configurations were not be determined. Galanganal (366), galanganols A (367), and B (368) were also found from rhizomes of A. galanga (L.) Willd. ${ }^{166}$ Compounds 361-367 exhibited significant cytotoxic activity against cancer Hela cells with $\mathrm{IC}_{50}$ values ranging from 1.5 to $5.29 \mu \mathrm{g} \mathrm{mL}^{-1}$. $^{165}$ Interestingly, 366 and 368 also inhibited NO production in mouse peritoneal macrophages. ${ }^{166}$ Galanganol C (369) was obtained from rhizomes of $A$. galanga (L.) Willd as a NO production inhibitor. ${ }^{\mathbf{1 6 6}}$ The whole plant of $A$. conchigera yielded three unusual sesquineolignans, conchignans A-C (370-372) bearing a tetrahydropyrane ring. ${ }^{167}$ 7-Methoxycoumarin (373) is a coumarin known from A. calcarata Rosc. $^{85}$

Citrusin B (374) and 2,3-dihydro-2-(4- $\beta$-D-glucopyranosyl-3methoxyphenyl)-3-hydroxymethyl-7-hydroxy-5-

benzofranpropanol (375) were the only two lignan glycosides isolated from leaves of $A$. speciosa. ${ }^{168}$

\section{Flavonoids}

To date, 71 flavanoides (Fig. 7) were isolated from the Alpinia species, including seven flavones (376-382), 14 flavonols (383396), four flavanones (397-400), seven flavanonols (401-407), two dihydrochalcones (408 and 409), 13 chalcones (410-422), four flavanols (423-426), and 18 flavonoid glycosides (427-444), two flavonoid oligomers (445 and 446).

Tectochrysin (376) and chrysin (377) were isolated from $A$. oxyphylla Miq. and exhibited moderate anti-inflammatory activities against LPS-induced NO production in RAW264.7 macrophage cells. ${ }^{169}$ Both A. bracteata and A. officinarum Hance produced apigenin (378), which displayed moderate activity on scavenging DPPH free radicals $\left(\mathrm{EC}_{50}=90 \pm 1.5 \mu \mathrm{M}\right) .{ }^{170} \mathrm{~A}$. galanga (L.) Willd was the source of 379-381 and A. tonkinensis Gagnep. produced 5-hydroxy-3', $4^{\prime}, 7$-trimethoxy flavanone (382). ${ }^{102,171}$ Kaempferol-3,4'-dimethylether (383) was afforded by A. sichuanensis Z. Y. Zhu. ${ }^{52}$ Galangin (384) and kaempferide (385) were the major flavonols distributed in several plants of Alpinia, both of which exhibited inhibitory against penicillinase and potent antioxidant activities. ${ }^{113,172}$ In addition, galangin effectively inhibited the TPA-induced invasion and migration of HepG2 cells at concentrations of $2.5-5 \mu \mathrm{M} .{ }^{173}$ In 2001 , a review summarized anti-genotoxic activity of galangin and demonstrated that galangin was a promising candidate for cancer chemoprevention. ${ }^{\mathbf{1 7 4}}$ Investigation on the whole plant of $A$. sichuanensis Z. Y. Zhu provided kaempferol (386). ${ }^{52}$ From A. speciosa, A. galanga (L.) Willd, A. katsumadai Hayata, and A. tonkinensis Gagnep., 3-methoxykaempferol (387) was isolated. ${ }^{175-178}$ While A. flabellata Ridley, A. oxyphylla, and A. 
tonkinensis Gagnep. yielded 3,5-dihydroxy-7,4'-dimethoxyflavone (388). ${ }^{113,161,171}$ Izalpinin (389) from different parts of $A$. oxyphylla Miq. was a NO production inhibitor and exhibited potent antioxidant activity. ${ }^{113,176}$ From rhizomes of $A$. officinarum, 3-methylethergalangin (390) was identified as an inhibitor of pancreatic lipase with an $\mathrm{IC}_{50}$ value of $1.3 \mathrm{mg} \mathrm{mL}{ }^{-1} \cdot{ }^{179}$ Compounds 391-395 were mainly obtained from A. tonkinensis Gagnep. ${ }^{111}$ 5-Hydroxy-3,7,4'-trimethoxyflavone (396) was yielded by leaves of A. flabellata Ridley. ${ }^{180}$ Pinocembrin (397) and alpinetin (398) were distributed in several Alpinia species and both showed antiemetic activities. ${ }^{121,181}$ In addition, 397 also demonstrated several bioactivities, such as cytotoxicity (on human T4 lymphoblastoid cancer cells), ${ }^{182}$ anti-inflammation, ${ }^{169}$ and antiplatelet aggregation $e t c .{ }^{183}$ While, 398 was a PAF receptor binding inhibitor. ${ }^{184} 7,4^{\prime}$-Dihydroxy-5-methoxy flavanone (399), pinostrobin (400) were reported from several species. ${ }^{116,118,128,182}$ Pinobanksin (401), $(2 R, 3 S)$-pinobaksin-3cinnamate (402), and 3-O-acetylpinobanksin (403) were mainly obtained from A. galanga (L.) Willd and A. katsumadai Hayata. ${ }^{176,177,185}$ Compound 402 showed potent neuroprotective effect against PC12 cells. ${ }^{177,186}$ Leaves of A. flabellata Ridley provided 404 and 405. ${ }^{180}$ Dihydrokaempferol (406) were isolated from A. oxyphylla. ${ }^{169}$ Both A. japonica (Thunb.) Miq. and A. galanga (L.) Willd were sources for alpinone (407). ${ }^{176,187}$ From seeds of A. katsumadai Hayata, a dihydrochalcone uvangoletin (408) was isolated. ${ }^{108} A$. speciosa $\mathrm{K}$. Schum. and A. formosana afforded another dihydrochalcone, dihydroflavokawin B (409). ${ }^{81,188}$ Flavokawin B (410) was isolated from several plants and showed strong cytotoxicity against human T4 lymphoblastoid cancer cells $\left(\mathrm{IC}_{50}=6.5 \mu \mathrm{M}\right)$ and anti-inflammatory activity. ${ }^{182,189}$ Cardamomin (411) distributed in many Alpinia species ${ }^{\mathbf{1 0 0 , 1 1 8 , 1 2 3 , 1 8 8 , 1 9 0}}$ and exhibited extensive bioactivities including death receptor 5 (DR5) promotor, ${ }^{175}$ antimicrobial, ${ }^{191}$ antiemetic, ${ }^{121}$ anticoagulation, ${ }^{183}$ and anti-inflammation. ${ }^{128}$ Interestingly, it also protected septic mice from acute lung injury by preventing endothelial barrier dysfunction. ${ }^{192} 2^{\prime}, 3^{\prime}, 4^{\prime}, 6^{\prime}$-Tetrahydroxychalcone (412), which was obtained from A. rafflesiana Wall.ex.Bak., was potently active to DPPH free radical scavenging $\left(\mathrm{IC}_{50}=55 \mu \mathrm{M}\right) \cdot{ }^{128}$ Rhizomes of A. pricei Hayata yielded $2^{\prime}, 4^{\prime}, 6^{\prime}$ trimethoxychalcone (413) and pinostrobin chalcone (414). ${ }^{189}$ Compounds 415-417 were isolated from the seeds of $A$. blepharocalyx K. Schum., ${ }^{116,117}$ while helichrysetin (415) was also found in A. katsumadai Hayata. ${ }^{108}$ Pinocembrin chalcone (418) and $4^{\prime}, 6^{\prime}$-dimethylchalconaringenin (419) were provided by $A$. katsumadai Hayata and A. pinnanensis T. L. Wu et Senjen, respectively. ${ }^{118,181}$ Compound $\mathbf{4 1 8}$ was also isolated from $A$. platychilus. ${ }^{193}$ Galanganones A-C (420-422) were three novel chalcones bearing a long-chain alkylphenol from A. galanga. ${ }^{194}$ Whilst $A$. katsumadai Hayata and A. zerumbet (Pers.) B. L. Burttet Smith. provided (+)-catechin (423). ${ }^{195,196}$ Epicatechin (424) and galloepicatechin (425) were yielded by A. oxymitra K. Schum. ${ }^{75}$ (+)-Epicatechin (426) was isolated from A. speciosa K. Schum. and displayed antioxidant activity. ${ }^{197}$ Kaempferide-3-O- $\beta$-Dglucoside (427) from A. officinarum Hance had an weak inhibitory activity against penicillinase. ${ }^{172}$ Study on A. speciosa $\mathrm{K}$. Schum. lead to the isolation of 428-432. ${ }^{198}$ Quercetin 3-O-robinobioside (433) and galangoflavonoside (434) were obtained from A. katsumadai Hayata and A. galanga (L.) Swartz., respectively. ${ }^{196,199}$ Compounds 435-437 from A. densespicata Hayata exhibited moderate NO inhibitory activities. ${ }^{103}$ Compounds 438-440 were obtained from the seeds of $A$. katsumadai Hayata and isorhamnetin-3-O- $\beta$-D-galactosyl-( $6 \rightarrow$ 1)- $\alpha$-L-rhamnoside (441) was isolated from rhizomes of A. tonkinensis Gagnep. ${ }^{51,196}$ Leaves of A. zerumbet (Pers.) B. L. Burttet Smith. contained rutin (442) and kaempferol-3-O-rutinoside (443) ${ }^{195}$ The whole plant of A. sichuanensis Z. Y. Zhu yielded hesperidin (444). ${ }^{52}$ Two pairs of enantiomers of flavonoidoligomers (445a and 445b, 446a and 446b) were found from rhizomes of A. platychilus. The compounds mixture of $\mathbf{4 4 6 a}$ and $\mathbf{4 4 6 b}$ showed anticoagulant activity on the prolongation of both prothrombin times (PT) and the thrombin times (TT) with a dose-effect relationship at 6.25$100 \mathrm{mM}^{193}$

\section{Phenolics}

A total number of 66 phenolics (447-512) were obtained from Alpinia species (Fig. 8). [Di-(p-hydroxy-cis-styryl)]methane (447) was obtained from A. galanga (L.) Willd. ${ }^{200}$ Whist alpininone (448) was isolated from A. gagnepainii K. Schum. with antibacterial effect against $E$. coli, B. subtilis, and $S$. aureus with the same MIC value of $12.5 \mu \mathrm{g} \mathrm{mL}{ }^{-1}{ }^{191}(1 E, 4 Z)-5$-Hydroxy-1phenylhexa-1,4-dien-3-one (449) and 2-propenal, 3-[4(acetyloxy)-3-methoxyphenyl] (450) were provided by $\mathrm{A}$. katsumadai Hayata and A. galanga (L.) Willd, respectively. ${ }^{86,108}$ From A. sichuanensi and A. oxyphylla, dibutyl phthalate (451) was isolated..$^{52,201}$ Two compounds named as $(E)$-p-coumaryl alcohol (452) and (E)-p-coumaryl alcohol $\gamma$-O-methyl ether (453) exhibited potent inhibitory activities against the autoxidation of methyl linoleate in bulk phase. ${ }^{163}$ In addition, compound 453 exerted potent cytotoxic activity against the SNU638 cells with $\mathrm{IC}_{50}$ value of $1.62 \mu \mathrm{g} \mathrm{mL} \mathrm{m}^{-1}$. $^{202}$

A. galanga (L.) Willd and A. conchigera Griff. produced trans$p$-hydroxycinnamaldehyde (454) and trans-p-hydroxycinnamyl acetate (455). ${ }^{170,203}$ Compound 454 displayed weak antiallergic effect, ${ }^{204}$ and NO production inhibitory activities $\left(\mathrm{IC}_{50}=20\right.$ $\mu \mathrm{M}),{ }^{166}$ and 455 exerted no inhibitory activity towards Staphylococcus aureus strain VISA (MIC $=203 \mathrm{mM}){ }^{82}$ trans- $p$-Coumaryl alcohol (456) was a weak NO production inhibitor from A. galanga $\left(\mathrm{L}\right.$.) Willd $\left(\mathrm{IC}_{50}=72 \mu \mathrm{M}\right) \cdot{ }^{166}$ trans-p-Coumaryl diacetate (457) from A. galanga showed a number of bioactivities, including anti-allergy, ${ }^{204}$ efflux pump inhibition, ${ }^{205} \mathrm{NO}$ production inhibition, ${ }^{166}$ xanthine oxidase inhibition, ${ }^{206}$ antileishmania, ${ }^{164}$ cytotoxicity, ${ }^{203}$ and antibacteria. ${ }^{82}$ trans-p-Acetoxycinnamyl alcohol (458), trans-p-hydroxylcinnamaldehyde acetate (459), and $p$-coumaric acid (460) were obtained from rhizomes of $A$. galanga (L.) Willd. ${ }^{164,205}$ In addition, compound $\mathbf{4 6 0}$ was also distributed in $A$. galanga (L.) Willd, ${ }^{164}$ A. sichuanensis Z. Y. Zhu, ${ }^{52}$ A. speciosa,${ }^{198} A$. blepharocalyx K. Schum. ${ }^{116}$ and A. oxyphylla ${ }^{169}$ Both A. formosana and $A$. speciosa $\mathrm{K}$. Schum. were sources of methyl trans-cinnamate (461). ${ }^{81,188}$ Seeds of A. blepharocaly yielded methyl $p$-hydroxycinnamate (462) and methyl $p$-hydroxycinnamyl ketone (463). ${ }^{116}$ From rhizomes of A. galanga (L.) Willd, 12 compounds (464-475) were obtained. . $3,82,203,207$ Among them, 1S-1'-acetoxychavicol acetate (464) and 1-acetoxyeugenol acetate (465) were the most 
abundant phenylpropanoids presented in A. galanga (L.) Swartz., A. officinarum Hance, and A. conchigera Griff. They were reported to have anti-ulcer, ${ }^{24}$ antileishmanial, ${ }^{\mathbf{1 6 4}}$ and antitumor bioactivities, ${ }^{33,202,208}$ Furthermore, 464 also showed antiallergic, ${ }^{204}$ efflux pump inhibitory, ${ }^{205}$ NO production inhibitory, ${ }^{166}$ xanthine oxidase inhibitory, ${ }^{206}$ gastroprotective ${ }^{209}$ anti-HIV ${ }^{210}$ anti-cancer, ${ }^{86}$ antibacterial, ${ }^{30,211}$ plant growth-inhibitory and fungal growthinhibitory activities. ${ }^{212}$ Two compounds, methyleugenol (466) and hydroxychavicol acetate (467), were isolated from A. galanga (L.) Willd. ${ }^{82,164,166,204,211}$ It was demonstrated that 467, a chavicol acetate analogue, suppressed T-bet expression in Th cells. ${ }^{\mathbf{2 1 1}}$ Besides, 467 also showed weak antibacterial activity against Staphylococcus aureus strain VISA (MIC $=0.8 \mathrm{mM}) .{ }^{82}$ trans-Coniferyl diacetate (468) was proved to be a xanthine oxidase inhibitor. $^{206}$ Three new phenolics 469,470 , and 471 , along with four known ones 472-475 were also yielded by A. galang..$^{33,203,207}$ Chavicol acetate (476) and $1^{\prime} S$-acetoxyeugenol acetate (477) were two known phenolics found from $A$. conchigera Griff. ${ }^{82}$ Compound 477 possessed antibacterial, ${ }^{82}$ xanthine oxidase inhibitory, ${ }^{206}$ gastroprotective, ${ }^{209}$ and anti-cancer activities.$^{86}$ Investigation on leaves of A. flabellata Ridley provided 478-480, with strong antibacterial activities against Staphylococcus aureus. ${ }^{\mathbf{1 6 1 , 1 6 2 , 1 8 0}}$ Compounds 481489 were nine phenolic acids isolated from several Alpinia species..$^{52,89,122,180,198,213,214}$ Protocatechuic acid (489) showed potent neuroprotective effect on $\mathrm{MPP}^{+}$-induced neurotoxicity and $\mathrm{H}_{2} \mathrm{O}_{2}$ induced oxidative damage in PC12 cells. ${ }^{215-219}$ In addition, it also exerted anti-aging effect on spleen and liver antioxidative system of senescent mice. ${ }^{31}$ 4-Hydroxybenzaldehyde (490), isolated from A. sichuanensis Z. Y. Zhu, A. blepharocalyx K. Schum., A. bracteata, and $A$. galanga (L.) Willd, ${ }^{52,116,117,166,170}$ didn't show any DPPH radical-scavenging activity. Instead, it exhibited inhibitory activity on xanthine oxidase $\left(\mathrm{IC}_{50}=19.6 \mu \mathrm{M}\right) .{ }^{170,206}$ Compounds 491-496 were provided by several Alpinia plants..$^{52,84,137,167,220}$

Ethyl 4- $O$-feruloyl- $\beta$-glucopyranoside (497) and 4-hydroxy-3methoxyphenyl 4- $O$-feruloyl- $\beta$-glucopyranoside (498) were two new glucoside esters of ferulic acid from rhizomes of $A$. speciosa, both of which showed antioxidant activities. ${ }^{197}$ Investigation on rhizomes of A. officinarum Hance yielded 499-506. ${ }^{55}$ While from rhizomes of $A$. bracteata, a new phenolic glycoside (507) was isolated and showed moderate antioxidant activity on scavenging DPPH free radicals $\left(\mathrm{EC}_{50}=169 \pm 4.8 \mu \mathrm{M}\right) .{ }^{170}$ Leaves of $A$. speciosa K. Schum. provided coniferin (508) and syringin (509). ${ }^{168}$

Dihydro-5,6-dehydrokawain (510) and 5,6-dehydrokawain (511) were major chemical constituents in several Alpinia species. ${ }^{\mathbf{8 1 , 1 0 0 , 1 2 8 , 1 7 5 , 1 8 8 , 2 2 1}}$ They showed antiulcerogenic, antithrombotic, ${ }^{195}$ antifungal, ${ }^{191}$ anti-obesity, ${ }^{222}$ and plant growth inhibitory activities. ${ }^{223}$ Recently, it was reported that they could strongly inhibit HIV-1 integrase with respective IC $_{50}$ values of 4.4 and $3.6 \mu \mathrm{g} \mathrm{mL}^{-1}$. In addition, they exhibited mixed type of inhibition against neuraminidase with both $\mathrm{IC}_{50}$ values of 25 $\mu \mathrm{M} .{ }^{95}$ Furthermore, $\mathbf{5 1 1}$ was also reported as a slow and timedependent reversible inhibitor of neuraminidase, a moderated antioxidant, a strong inhibitor of skin diseases-related enzymes, and strong antiplatelet inhibitor. ${ }^{\mathbf{9 5 , 1 2 7 , 2 2 4}}$ Interestingly, a dimer of 5,6-dehydrokawain, AS-II (511a), was an artifact formed by photo-irradiation during the isolation procedure of A. speciosa
K. Schum. leaves. ${ }^{223}$ 4-Hydroxy-5,6-dehydrokawain (512) was an $\alpha$-pyrone isolated from A. blepharocalyx K. Schum. It displayed antiproliferative activity against murine colon 26-L5 carcinoma and human HT-1080 fibrosarcoma with $\mathrm{ED}_{50} 20.7$ and $20.1 \mu \mathrm{M}$, respectively. ${ }^{116,117}$ It also showed inhibitory effect on platelet aggregation induced by collagen, arachidonic acid (AA), adenosine diphosphate, and ristocetin. ${ }^{96}$

\section{Steroids}

Seven steroids (Fig. 9) were isolated from Alpinia species including four cholestanes (513-516) and three sitosterol glycosides (517-519). ${ }^{27,52,89,116,118,225}$ As it is the same in plants of the other genera, $\beta$-sitosterol (513) and stigmasterol (514) were also widely distributed in Alpinia species..$^{52,82,89,118,178,191,226-228} \beta$ Sitosterol-3-O- $\beta$-D-6-palmitoylglucoside (518) showed potent antiemetic activity induced by $\mathrm{CuSO}_{4} \cdot{ }^{27}$

\section{Alkaloids}

Officinaruminane A (520) and officinine B (521), two alkaloids of bi-diarylheptanoid connecting by a pyridine ring were contributed by rhizomes of $A$. officinarum Hance. ${ }^{131,157}$ A study on seeds of A. katsumadai Hayata afforded another six alkaloids (522-527) (Fig. 9). ${ }^{108,196}$

\section{Stilbenes}

Six stilbenes, 528-533 (Fig. 9), were all isolated from aerial parts of A. katsumadai Hayata. ${ }^{29,121}$

\section{Others}

One esters (534) and three fatty acids, 535-537, were isolated from several Alpinia species. ${ }^{64,227-229}(S)$-2-Pentanol-2-O- $\beta$-D-glucopyranoside (538), which showed inhibitory effect on NO production from LPS-activated RAW264.7 macrophage cells, was obtained from fruits of $A$. oxyphylla. ${ }^{89}$ Two glycosides known as 3methyl-but-2-en-1-yl- $\beta$-D-glucopyranoside (539) and $n$-butyl- $\beta$-Dfructopyranoside (540) were isolated from $A$. officinarum Hance ${ }^{\mathbf{5 5 , 2 3 0}}$ While 541-544 were found in different Alpinia species (Fig. 9). ${ }^{\mathbf{2 4 5 1 , 1 0 8 , 1 9 6 , 2 0 1}}$ Interestingly, 5-hydroxymethylfurfural (544) exerted memory improvement activity against Alzheimer's disease $(\mathrm{AD})$ by mitigating the degree of neuronal damage. ${ }^{231}$

\section{Conclusions}

The number of publications on the chemical constituents and their bioactivities for Alpinia species from 1955 to 2015 are shown in Fig. 10. Before 1999, fewer investigations (less than five per year, except six in 1987) were performed on this genus. However, after 2009, there were more than 10 papers published for each year. In 2013, the number of published articles reached 26, indicating a growing interest in the genus of Alpinia.

Till 2015, investigations on chemical constitutes of the Alpinia species afforded a total of 544 compounds, including 207 terpenoids, 143 diarylheptanoids, 25 phenylpropanoids, 71 
Table 1 The number of new compounds isolated from each Alpinia species since 1955

\begin{tabular}{|c|c|c|c|c|c|c|}
\hline Sources of new compounds & Terpenoids & Diarylheptanoids & Lignans & Flavanoids & Phenolics & Sum \\
\hline A. blepharocalyx & - & 45 & - & - & - & 45 \\
\hline A. bracteata & - & - & - & - & 1 & 1 \\
\hline A. calcarata & 6 & - & 1 & - & - & 7 \\
\hline A. chinensis & 10 & - & - & - & - & 10 \\
\hline A. densibracteata & 1 & - & - & - & - & 1 \\
\hline A. formosana & 1 & - & - & - & - & 1 \\
\hline A. galanga & 4 & - & - & 5 & 4 & 13 \\
\hline A. gagnepainii & - & - & - & - & 1 & 1 \\
\hline A. intermedia & 8 & - & - & - & - & 8 \\
\hline A. japonica & 14 & - & - & - & - & 14 \\
\hline A. katsumadai & 2 & 29 & 1 & - & 1 & 33 \\
\hline A. pahangensis & 5 & - & - & - & - & 5 \\
\hline A. pinnanensis & - & 2 & - & - & - & 2 \\
\hline A. rafflesiana & - & - & - & 1 & - & 1 \\
\hline A. tonkinensis & 2 & - & - & - & - & 2 \\
\hline A. zerumbet (A. speciosa) & 2 & - & - & - & 2 & 4 \\
\hline Sum & 106 & 96 & 21 & 8 & 16 & 247 \\
\hline
\end{tabular}

flavanones, 66 phenolics, seven steroids, eight alkaloids, six stilbenes, and 11 others (Fig. 11). Among 207 terpenoids, 17 are monoterpenoids, 132 are sesquiterpenoids, 57 are diterpenoids, and the rest one is a triterpenoid. For sesquiterpenoids, eudesmanes and eremophilanes are undoubtedly predominant with 44 and 21 components, respectively. While for diterpenoids, almost all are labdanes.

Amongst 544 isolated compounds from the genus of Alpinia, 247 are new ones (Table 1), including 96 diarylheptanoids and 106 terpenoids. Obviously, diarylheptanoids, especially diarylheptane-flavonoids conjugates, are characteristic components for the genus of Alpinia. ${ }^{149}$

The crude extracts of Alpinia species and their chemical constituents were found to possess various biological activities. Mainly reported were antiemetic, ${ }^{26,27}$ antibacterial, ${ }^{29-31,37,82,232-236}$ antioxidant, ${ }^{127,237-239}$ anticancer, ${ }^{32-34,240-245}$ anti-inflammatory, ${ }^{189,246,247}$ insecticidal, ${ }^{36,164}$ and neuroprotective bioactivities. ${ }^{38,39,231,248-250}$

In addition, they also showed antiulcer, ${ }^{25}$ antiplatelet, ${ }^{\mathbf{1 1 7}, \mathbf{1 8 3}}$ hepatoprotective, ${ }^{251}$ and hypolipidemic effects. ${ }^{252}$ Meanwhile, evidences showed that ethanol extract of A. galangal can retard lipid oxidation for minced beef, indicating a great potential utility for food storage. ${ }^{8}$ What should be aroused considerable interest was the promising anticancer and hepatoprotective properties, which could be a great potential to be developed as herbal medicines.

Although there are about 230 species for the Alpinia genus, only 35 were investigated for their chemical constituents and bioactivities (Fig. 12), because A. jianganfeng T. L. Wu includes Alpinia sichuanensis Z. Y. Zhu, and A. zerumbet (Pers.) B. L. Burtt \& R. M. Sm. includes A. speciosa K. Schum. according to The Plant List. Among these species, A. galanga, A. oxyphylla, A. officinarum, and A. katsumadai are four most studied plants with referenced papers of 43,40,32, and 23, respectively. While for the rest of 31 species, only very fewer articles were published, most of which were less than five. As a matter of fact, there was even only one paper published for 18 species. Although this genus contributed a diverse array of bioactive compounds, the potential of Alpinia species remains virtually untapped. Thus, much attention should be paid to Alpinia species on further phytochemical and pharmacological studies, which would produce structurally interesting and biologically active compounds with potential use in agricultural and medicinal applications. In addition, although most of Alpinia species were also used as edible plants, the nutritious components and their effects were seldom investigated, which could be a hotspot in the near future.

\section{Acknowledgements}

The project was supported by National Natural Science Foundation of China (41176148, 21372233, 21202080).

\section{Notes and references}

1 D. Wu and L. Kai, in Flora of China, ed. Z. Y. Wu, Science Press, Beijing, 2000, vol. 24, p. 333.

2 T. Wu, Redai Yaredai Zhiwu Xuebao, 1994, 2, 1.

3 J. Jonczyk, Acta Pol. Pharm., 1970, 27, 155.

4 K. D. Kobayashi, J. McEwen and A. J. Kaufman, Ornamentals and Flowers, 2007, 37.

5 C. Wang, C. Xu, A. Tian, S. Fu and C. Wang, Color. Technol., 2013, 129, 32. 
6 X. Yang and R. G. Eilerman, J. Agric. Food Chem., 1999, 47, 1657.

7 H. Morita and H. Itokawa, Chem. Lett., 1986, 15, 1205.

8 P. B. Cheah and N. H. Abu Hasim, J. Sci. Food Agric., 2000, 80, 1565.

9 P. Pripdeevech, N. Nuntawong and S. Wongpornchai, Chem. Nat. Compd., 2009, 45, 562.

10 J. Qiu, CN. Pat., CN101711590A, 2010.

11 Z. Wei, CN. Pat., CN1385106A, 2002.

12 L. Xu and X. Gao, CN. Pat., CN1481713A, 2004.

13 H. Yan, CN. Pat., CN102084999A, 2011.

14 H. Yang, CN. Pat., CN103156116A, 2013.

15 B. Zhu, CN. Pat., CN101380121A, 2009.

16 J. Wang, W. Hou and Z. Zhang, CN. Pat., CN104893910A, 2015.

17 Z. Cheng, CN. Pat., CN102429267A, 2012.

18 Y. Dou, CN. Pat., CN101088380A, 2007.

19 H. Zhao, CN. Pat., CN1138960A, 1997.

20 L. X. Du, Z. T. Jiang and R. Li, Zhongguo Tiaoweipin, 2012, $37,22$.

21 H. Itokawa, H. Morita, K. Watanabe, S. Mihashi and Y. Iitaka, Chem. Pharm. Bull., 1985, 33, 1148.

22 H. Itokawa, H. Morita, I. Midorikawa, R. Aiyama and M. Morita, Chem. Pharm. Bull., 1985, 33, 4889.

23 B. Roy and A. Swargiary, J. Parasit. Dis., 2009, 33, 48.

24 S. Mitsui, S. Kobayashi, H. Nagahori and A. Ogiso, Chem. Pharm. Bull., 1976, 24, 2377.

25 M. A. Al-Yahya, S. Rafatullah, J. S. Mossa, A. M. Ageel, M. S. Al-Said and M. Tariq, Phytother. Res., 1990, 4, 112.

26 Y. Yang, K. Kinoshita, K. Koyama, K. Takahashi, T. Tai, Y. Nunoura and K. Watanabe, Nat. Prod. Sci., 1999, 5, 20.

27 D. Shin, K. Kinoshita, K. Koyama and K. Takahashi, J. Nat. Prod., 2002, 65, 1315.

28 Y. Yang, K. Kinoshita, K. Koyama, K. Takahashi, S. Kondo and K. Watanabe, Phytomedicine, 2002, 9, 146.

29 K. Rao, B. Ch, L. Narasu and A. Giri, Appl. Biochem. Biotechnol., 2010, 162, 871.

30 P. Niyomkam, S. Kaewbumrung, S. Kaewnpparat and P. Panichayupakaranant, Pharm. Biol., 2010, 48, 375.

31 X. Zhang, G. F. Shi, X. Z. Liu, L. J. An and S. Guan, Cell Biochem. Funct., 2011, 29, 342.

32 K. S. Chun, Y. Sohn, H. S. Kim, O. H. Kim, K. K. Park, J. M. Lee, J. Lee, J. Y. Lee, A. Moon, S. S. Lee and Y. J. Surh, Mutat. Res., 1999, 428, 49.

33 H. Itokawa, H. Morita, T. Sumitomo, N. Totsuka and K. Takeya, Planta Med., 1987, 53, 32.

34 S. Samarghandian, M. A. R. Hadjzadeh, J. Afshari and M. Hosseini, BMC Complementary Altern. Med., 2014, 14, 1.

35 R. Rajasekar, K. Manokaran, N. Rajasekaran, G. Duraisamy and D. Kanakasabapathi, J. Diabetes Metab. Disord., 2014, 13, 1.

36 Y. M. Chang, C. T. Tsai, C. C. R. Wang, Y. S. Chen, Y. M. Lin, C. H. Kuo, B. S. Tzang, R. J. Chen, F. J. Tsai and C. Y. Huang, Biosci., Biotechnol., Biochem., 2013, 77, 229.

37 K. Klahan, N. Nantapong and N. Chudapongse, Planta Med., 2011, 77, PM27.

38 S. H. Shi, X. Zhao, A. J. Liu, B. Liu, H. Li, B. Wu, K. S. Bi and Y. Jia, Physiol. Behav., 2015, 139, 13.
39 X. Z. Li, S. N. Zhang, S. M. Liu and F. Lu, Fitoterapia, 2013, 84, 273.

40 D. P. de Sousa, P. de Almeida Soares Hocayen, L. N. Andrade and R. Andreatini, Molecules, 2015, 20, 18620.

41 H. Lv and G. She, Nat. Prod. Commun., 2010, 5, 1687.

42 D. Kaushik, J. Yadav, P. Kaushik, D. Sacher and R. Rani, Zhongxiyi Jiehe Xuebao, 2011, 9, 1061.

43 J. W. Nam and E. K. Seo, Nat. Prod. Commun., 2012, 7, 795.

44 P. Chen, P. P. Wang, Z. Z. Jiao and L. Xiang, Xiandai Yaowu Yu Linchuang, 2013, 28, 617.

45 M. A. Rahman and M. S. Islam, Pharmacogn. Rev., 2015, 9, 55. 46 E. W. Chan and S. K. Wong, J. Integr. Med., 2015, 13, 368. 47 T. D. Xuan and R. Teschke, Molecules, 2015, 20, 16306.

48 S. Z. Hua, J. G. Luo, X. B. Wang, J. S. Wang and L. Y. Kong, Bioorg. Med. Chem. Lett., 2009, 19, 2728.

49 K. S. Ngo and G. D. Brown, Phytochemistry, 1998, 47, 1117. 50 L. K. Sy and G. D. Brown, Phytochemistry, 1997, 45, 537.

51 J. Zhang and L. Y. Kong, J. Asian Nat. Prod. Res., 2004, 6, 199.

52 D. Liu, W. Qu and J. Y. Liang, Biochem. Syst. Ecol., 2013, 46, 127.

53 J. J. Xu, N. H. Tan, Y. S. Chen, X. L. Pan, G. Z. Zeng, H. J. Han, C. J. Ji and M. J. Zhu, Helv. Chim. Acta, 2009, 92, 1621.

54 Y. Someya, A. Kobayashi and K. Kubota, Biosci., Biotechnol., Biochem., 2001, 65, 950.

55 T. N. Ly, R. Yamauchi, M. Shimoyamada and K. Kato, J. Agric. Food Chem., 2002, 50, 4919.

56 U. Grienke, M. Schmidtke, J. Kirchmair, K. Pfarr, P. Wutzler, R. Dürrwald, G. Wolber, K. R. Liedl, H. Stuppner and J. M. Rollinger, J. Nat. Med., 2010, 53, 778.

57 M. Morita, H. Nakanishi, H. Morita, S. Mihashi and H. Itokawa, Chem. Pharm. Bull., 1996, 44, 1603.

58 M. Miyazawa, Y. Nakamura and Y. Ishikawa, J. Agric. Food Chem., 2000, 48, 3639.

59 P. Chen, P. P. Wang, Z. Z. Jiao and L. Xiang, Helv. Chim. Acta, 2014, 97, 388.

60 P. Chen, L. Qu, L. Tian, P. P. Wang and L. Xiang, Helv. Chim. Acta, 2013, 96, 1163.

$61 \mathrm{H}$. Itokawa, H. Morita, T. Kobayashi, K. Watanabe and Y. Iitaka, Chem. Pharm. Bull., 1987, 35, 2860.

62 S. Ando, H. Matsuda, T. Morikawa and M. Yoshikawa, Bioorg. Med. Chem., 2005, 13, 3289.

63 X. Luo, J. Yu, L. Xu, K. Li, P. Tan and J. Feng, Yaoxue Xuebao, 2000, 35, 204.

64 L. Hou, X. X. Lu, B. B. Xie, W. H. Huang, J. G. Yu and B. L. Guo, Tianran Chanwu Yanjiu Yu Kaifa, 2013, 25, 878.

65 J. Luo, X. Lv, X. Wang and L. Kong, Phytochem. Lett., 2012, 5, 134.

66 J. J. Xu, N. H. Tan, J. Xiong, A. H. Adebayo, H. J. Han, G. Z. Zeng, C. J. Ji, Y. M. Zhang and M. J. Zhu, Chin. Chem. Lett., 2009, 20, 945.

67 D. H. Park, J. W. Lee, Q. Jin, W. K. Jeon, M. K. Lee and B. Y. Hwang, Bull. Korean Chem. Soc., 2014, 35, 1565.

68 X. Q. Lv, J. G. Luo, X. B. Wang, J. S. Wang, J. Luo and L. Y. Kong, Chem. Pharm. Bull., 2011, 59, 402.

69 H. Itokawa, H. Morita and K. Watanabe, Chem. Pharm. Bull., 1987, 35, 1460. 
70 H. Itokawa, K. Watanabe, S. Mihashi and Y. Iitaka, Chem. Pharm. Bull., 1980, 28, 681.

71 B. Jiang, W. J. Wang, M. P. Li, X. J. Huang, F. Huang, H. Gao, P. H. Sun, M. F. He, Z. J. Jiang, X. Q. Zhang and W. C. Ye, Bioorg. Med. Chem. Lett., 2013, 23, 3879.

72 O. Muraoka, M. Fujimoto, G. Tanabe, M. Kubo, T. Minematsu, H. Matsuda, T. Morikawa, I. Toguchida and M. Yoshikawa, Bioorg. Med. Chem. Lett., 2001, 11, 2217.

73 J. Xu, C. Ji, Y. Zhang, J. Su, Y. Li and N. Tan, Bioorg. Med. Chem. Lett., 2012, 22, 1660.

74 J. J. Xu, N. H. Tan, G. Z. Zeng, H. J. Han and Y. F. Peng, Chin. J. Nat. Med., 2010, 8, 6.

75 K. Jitsaeng, W. De-Eknamkul and B. Schneider, Rec. Nat. Prod., 2009, 3, 110.

76 S. M. Xu, X. J. Huang, Y. Wang and W. C. Ye, Zhongguo Tianran Yaowu, 2012, 10, 374.

77 L. Hou, G. Ding, B. L. Guo, W. H. Huang, X. J. Zhang, Z. Y. Sun and X. F. Shi, Molecules, 2015, 20, 1551.

78 H. Itokawa, H. Morita, K. Watanabe, A. Takase and Y. Iitaka, Chem. Lett., 1984, 13, 1687.

79 H. Itokawa, H. Morita, K. Osawa, K. Watanabe and Y. Iitaka, Chem. Pharm. Bull., 1987, 35, 2849.

$80 \mathrm{H}$. Itokawa, K. Watanabe, H. Morita, S. Mihashi and Y. Iitaka, Chem. Pharm. Bull., 1985, 33, 2023.

81 H. Itokawa, S. Yoshimoto and H. Morita, Phytochemistry, 1988, 27, 435.

82 A. N. Aziz, H. Ibrahim, D. Rosmy Syamsir, M. Mohtar, J. Vejayan and K. Awang, J. Ethnopharmacol., 2013, 145, 798.

83 Q. M. Li, J. G. Luo, X. B. Wang, M. H. Yang and L. Y. Kong, Fitoterapia, 2013, 86, 29.

84 J. Xu, N. Tan, G. Zeng, H. Han, H. Huang, C. Ji, M. Zhu and Y. Zhang, Zhongguo Zhongyao Zazhi, 2009, 34, 990.

85 L. Y. Kong, M. J. Qin and M. Niwa, J. Nat. Prod., 2000, 63, 939.

86 Q. H. Zeng, C. L. Lu, X. W. Zhang and J. G. Jiang, Food Funct., 2015, 6, 431.

87 H. Itokawa, H. Morita, K. Watanabe and Y. Iitaka, Chem. Lett. , 1984, 13, 451.

88 T. Morikawa, H. Matsuda, I. Toguchida, K. Ueda and M. Yoshikawa, J. Nat. Prod., 2002, 65, 1468.

89 Z. J. Qing, Y. Wang, L. Y. Hui, L. W. Yong, L. H. Long, D. J. Ao and P. L. Xia, Arch. Pharmacal Res., 2012, 35, 2143.

90 J. Xu, J. Su, Y. Li and N. Tan, Chem. Nat. Compd., 2013, 49, 457.

91 S. Ghosh, K. Indukuri, S. Bondalapati, A. K. Saikia and L. Rangan, Eur. J. Med. Chem., 2013, 66, 101.

92 S. Ghosh and L. Rangan, Appl. Biochem. Biotechnol., 2015, 175, 1477.

93 H. Morita and H. Itokawa, Planta Med., 1988, 54, 117.

94 J. Chompoo, A. Upadhyay, W. Kishimoto, T. Makise and S. Tawata, Food Chem., 2011, 129, 709.

95 A. Upadhyay, J. Chompoo, W. Kishimoto, T. Makise and S. Tawata, J. Agric. Food Chem., 2011, 59, 2857.

96 Y. Sivasothy, H. Ibrahim, A. S. Paliany, S. A. Alias, N. R. Md Nor and K. Awang, Planta Med., 2013, 79, 1775.

97 L. K. Sy and G. D. Brown, J. Nat. Prod., 1997, 60, 904.

98 H. Itokawa, M. Morita and S. Mihashi, Chem. Pharm. Bull., 1980, 28, 3452.
99 H. X. Xu, H. Dong and K. Y. Sim, Phytochemistry, 1996, 42, 149. 100 N. Nuntawong and A. Suksamrarn, Biochem. Syst. Ecol., 2008, 36, 661.

101 Q. M. Li, J. G. Luo, M. H. Yang and L. Y. Kong, Chem. Biodiversity, 2015, 12, 388.

102 V. S. Chauhan, M. Swapna and A. Singh, Int. J. Appl. Biol. Pharm. Technol., 2014, 5, 186.

103 Y. J. Kuo, P. C. Hsiao, L. J. Zhang, M. D. Wu, Y. H. Liang, H. O. Ho and Y. H. Kuo, J. Nat. Prod., 2009, 72, 1097.

104 S. Tesaki, H. Kikuzaki, S. Yonemori and N. Nakatani, J. Nat. Prod., 2001, 64, 515.

105 H. Itokawa, H. Morita, I. Katou, K. Takeya, A. J. Cavalheiro, R. C. B. de Oliveira, M. Ishige and M. Motidome, Planta Med., 1988, 54, 311.

106 Y. Sivasothy, H. Ibrahim, A. S. Paliany, S. A. Alias and K. Awang, Bioorg. Med. Chem. Lett., 2013, 23, 6280.

107 L. Y. Kong, M. J. Qin and M. Niwa, Planta Med., 2002, 68, 813.

108 X. B. Wang, C. S. Yang, S. Z. Hua and L. Y. Kong, Zhongguo Tianran Yaowu, 2010, 8, 419.

109 S. Y. Choi, M. H. Lee, J. H. Choi and Y. K. Kim, Biol. Pharm. Bull., 2012, 35, 2092.

110 H. J. Lee, J. S. Kim and J. H. Ryu, Planta Med., 2006, 72, 68.

111 R. J. Lin, C. M. Yen, T. H. Chou, F. Y. Chiang, G. H. Wang, Y. P. Tseng, L. Wang, T. W. Huang, H. C. Wang, L. P. Chan, H. Y. Ding and C. H. Liang, BMC Complementary Altern. Med., 2013, 13, 1.

112 N. Shoji, A. Umeyama, T. Takemoto and Y. Ohizumi, Planta Med., 1984, 50, 186.

113 Q. Y. Bian, S. Y. Wang, L. J. Xu, C. O. Chan, D. K. W. Mok and S. B. Chen, J. Asian Nat. Prod. Res., 2013, 15, 1094.

114 Q. Zhang, S. Luo, H. Wang and D. Fan, Zhongcaoyao, 1997, 28, 131.

115 S. Kadota, Y. Tezuka, J. K. Prasain, M. S. Ali and A. H. Banskota, Curr. Top. Med. Chem., 2003, 3, 203.

116 M. S. Ali, Y. Tezuka, S. Awale, A. H. Banskota and S. Kadota, J. Nat. Prod., 2001, 64, 289.

117 H. Dong, S. X. Chen, H. X. Xu, S. Kadota and T. Namba, J. Nat. Prod., 1998, 61, 142.

118 P. M. Giang, P. T. Son, K. Matsunami and H. Otsuka, Chem. Pharm. Bull., 2005, 53, 1335.

119 M. Kuroyanagi, T. Noro, S. Fukushima, R. Aiyama, A. Ikuta, H. Itokawa and M. Morita, Chem. Pharm. Bull., 1983, 31, 1544.

120 B. Groeblacher, O. Kunert and F. Bucar, Bioorg. Med. Chem., 2012, 20, 2701.

121 W. Z. Huang, C. F. Zhang, M. Zhang and Z. T. Wang, J. Chin. Chem. Soc., 2007, 54, 1553.

122 Y. Li, L. Yang, C. Wang, G. Chou and Z. Wang, Shanghai Zhongyiyao Daxue Xuebao, 2010, 24, 72.

123 Y. Y. Li, G. X. Chou and Z. T. Wang, Helv. Chim. Acta, 2010, 93, 382.

124 J. W. Nam, G. Y. Kang, A. R. Han, D. Lee, Y. S. Lee and E. K. Seo, J. Nat. Prod., 2011, 74, 2109.

125 J. W. Nam and E. K. Seo, Helv. Chim. Acta, 2013, 96, 1670.

126 C. Y. Lo, P. L. Liu, L. C. Lin, Y. T. Chen, Y. C. Hseu, Z. H. Wen and H. M. Wang, Sci. World J., 2013, 186505, DOI: $10.1155 / 2013 / 186505$. 
127 M. Habsah, N. H. Lajis, A. M. Ali, M. A. Sukari, Y. Y. Hin, H. Kikuzaki and N. Nakatani, Pharm. Biol., 2003, 41, 7.

128 H. Mohamad, F. Abas, D. Permana, N. H. Lajis, A. M. Ali, M. A. Sukari, T. Y. Y. Hin, H. Kikuzaki and N. Nakatani, Z. Naturforsch., C: J. Biosci., 2004, 59, 811.

129 B. B. Zhang, Y. Dai, Z. X. Liao and L. S. Ding, Fitoterapia, 2010, 81, 948.

130 H. M. Sirat, A. A. Rahman, H. Itokawa and H. Morita, Planta Med., 1996, 62, 188.

131 N. An, H. W. Zhang, L. Z. Xu, S. L. Yang and Z. M. Zou, Food Chem., 2010, 119, 513.

132 L. Zhao, W. Qu, J. Q. Fu and J. Y. Liang, Chin. J. Nat. Med., 2010, 8, 241.

133 N. An, Z. M. Zou, Z. Tian, X. Z. Luo, S. L. Yang and L. Z. Xu, Fitoterapia, 2007, 79, 27.

134 Y. Sun, K. Tabata, H. Matsubara, S. Kitanaka, T. Suzuki and K. Yasukawa, Planta Med., 2008, 74, 427.

135 D. Liu, Y. W. Liu, F. Q. Guan and J. Y. Liang, Fitoterapia, 2014, 96, 76.

136 Y. Sun, H. Matsubara, S. Kitanaka and K. Yasukawa, Helv. Chim. Acta, 2008, 91, 118.

137 G. J. Fan, Y. H. Kang, Y. N. Han and B. H. Han, Bioorg. Med. Chem. Lett., 2007, 17, 6720.

138 D. Liu, W. Qu, L. Zhao, F. Q. Guan and J. Y. Liang, Chin. J. Nat. Med., 2014, 12, 139.

139 Y. U. Kim, H. K. Son, H. K. Song, M. J. Ahn, S. S. Lee and S. K. Lee, Planta Med., 2003, 69, 72.

140 H. B. Lee, H. K. Lee, J. R. Kim and Y. J. Ahn, J. Korean Soc. Appl. Biol. Chem., 2009, 52, 367.

141 J. E. Shin, M. J. Han, M. C. Song, N. I. Baek and D. H. Kim, Biol. Pharm. Bull., 2004, 27, 138.

142 Z. Liu, M. M. Rafi, N. Zhu, K. Ryu, S. Sang, C. T. Ho and R. T. Rosen, ACS Symp. Ser., 2003, 851, 369.

143 F. Kiuchi, M. Shibuya and U. Sankawa, Chem. Pharm. Bull., 1982, 30, 2279.

144 K. Yasukawa, Y. Sun, S. Kitanaka, N. Tomizawa, M. Miura and S. Motohashi, J. Nat. Med., 2008, 62, 374.

145 S. I. Uehara, I. Yasuda, K. Akiyama, H. Morita, K. Takeya and H. Itokawa, Chem. Pharm. Bull., 1987, 35, 3298.

146 M. S. Ali, Y. Tezuka, A. H. Banskota and S. Kadota, J. Nat. Prod., 2001, 64, 491.

147 J. K. Prasain, Y. Tezuka, J. X. Li, K. Tanaka, P. Basnet, H. Dong, T. Namba and S. Kadota, Planta Med., 1999, 65, 196.

148 M. S. Ali, A. H. Banskota, Y. Tezuka, I. Saiki and S. Kadota, Biol. Pharm. Bull., 2001, 24, 525.

149 X. B. Wang, C. S. Yang, C. Zhang, J. Luo, M. H. Yang, J. G. Luo, W. Y. Yu and L. Y. Kong, Tetrahedron, 2014, 70, 8714.

150 J. Kumar Prasain, Y. Tezuka, J.-X. Li, K. Tanaka, P. Basnet, H. Dong, T. Namba and S. Kadota, J. Chem. Res., Synop., 1998, 22, DOI: 10.1039/A706250H.

151 J. Kumar Prasain, Y. Tezuka, J. Xin Li, K. Tanaka, P. Basnet, H. Dong, T. Namba and S. Kadota, Tetrahedron, 1997, 53, 7833.

152 Y. Tezuka, M. B. Gewali, M. S. Ali, A. H. Banskota and S. Kadota, J. Nat. Prod., 2001, 64, 208.
153 J. K. Prasain, J. X. Li, Y. Tezuka, K. Tanaka, P. Basnet, H. Dong, T. Namba and S. Kadota, J. Nat. Prod., 1998, 61, 212.

154 M. B. Gewali, Y. Tezuka, A. H. Banskota, M. S. Ali, I. Saiki, H. Dong and S. Kadota, Org. Lett., 1999, 1, 1733.

155 L. Zhao, J. Y. Liang, J. Y. Zhang and Y. Chen, Chin. Chem. Lett., 2010, 21, 194.

156 D. Liu, W. Qu, L. Zhao and J. Y. Liang, Chin. Chem. Lett., 2012, 23, 189.

157 L. Zhao, J. Y. Liang and W. Qu, Chem. Nat. Compd., 2012, 48, 836.

158 S. Kadota, J. K. Prasain, J. X. Li, P. Basnet, H. Dong, T. Tani and T. Namba, Tetrahedron Lett., 1996, 37, 7283.

159 Y. Tezuka, M. S. Ali, A. H. Banskota and S. Kadota, Tetrahedron Lett., 2000, 41, 5903.

160 Y. Yang, K. Kinoshita, K. Koyama, K. Takahashi, T. Tai, Y. Nunoura and K. Watanabe, J. Nat. Prod., 1999, 62, 1672.

161 S. Tesaki, H. Kikuzaki, S. Tanabe, M. Watanabe and N. Nakatani, ITE Lett. Batteries, New Technol. Med., 2001, 2, 106.

162 H. Kikuzaki, S. Tesaki, S. Yonemori and N. Nakatani, Phytochemistry, 2001, 56, 109.

163 T. N. Ly, M. Shimoyamada, K. Kato and R. Yamauchi, J. Agric. Food Chem., 2003, 51, 4924.

164 A. Kaur, R. Singh, C. S. Dey, S. S. Sharma, K. K. Bhutani and I. P. Singh, Indian J. Exp. Biol., 2010, 48, 314.

165 J. J. Xu, G. Z. Zeng, S. C. Yang, Y. Shen and N. H. Tan, Fitoterapia, 2013, 91, 82.

166 T. Morikawa, S. Ando, H. Matsuda, S. Kataoka, O. Muraoka and M. Yoshikawa, Chem. Pharm. Bull., 2005, 53, 625.

167 J. J. Xu, H. M. Zhao, Y. Shen, J. H. Chen, Y. Li, N. H. Tan and S. C. Yang, J. Asian Nat. Prod. Res., 2013, 15, 833.

168 T. Obata, A. Sawabe, M. Morita, N. Yamashita and Y. Matsubara, J. Jpn. Oil Chem. Soc., 1995, 44, 1012.

169 N. Wei, Y. Wang, H. F. Li, J. Q. Zhang and Y. H. Li, Chem. Nat. Compd., 2013, 49, 934.

170 L. Liu, J. G. Luo and L. Y. Kong, Chem. Nat. Compd., 2012, 48, 785.

171 J. Zhang, Q. H. Guo and L. Y. Kong, Zhongguo Zhongyao Zazhi, 2003, 28, 41.

172 G. Eumkeb, S. Siriwong, S. Phitaktim, N. Rojtinnakorn and S. Sakdarat, J. Appl. Microbiol., 2012, 112, 55.

173 S. T. Chien, M. D. Shi, Y. C. Lee, C. C. Te and Y. W. Shih, Cancer Cell Int., 2015, 15, 1.

174 M. Y. Heo, S. J. Sohn and W. W. Au, Mutat. Res., 2001, 488, 135.

175 T. Ohtsuki, H. Kikuchi, T. Koyano, T. Kowithayakorn, T. Sakai and M. Ishibashi, Bioorg. Med. Chem., 2009, 17, 6748.

176 M. Q. Bian, H. Q. Wang, J. Kang, R. Y. Chen, Y. F. Yang and H. Z. Wu, Yaохue Xuebao, 2014, 49, 359.

177 B. R. Xin, S. J. Ren and J. Li, Zhongguo Zhongyao Zazhi, 2014, 39, 2674.

178 J. Zhang, X. He, J. Gao and L. Kong, Zhongguo Yaoxue Zazhi, 2003, 38, 502.

179 J. E. Shin, M. J. Han and D. H. Kim, Biol. Pharm. Bull., 2003, 26, 854. 
180 H. Kikuzaki and S. Tesaki, J. Nat. Prod., 2002, 65, 389.

181 X. Xiao, X. Si, X. Tong and G. Li, Sep. Purif. Technol., 2011, 81, 265.

182 N. A. Mustahil, M. A. Sukari, A. B. Abdul, N. A. Ali and G. E. C. Lian, Pak. J. Pharm. Sci., 2013, 26, 391.

183 I. Jantan, S. M. Raweh, H. M. Sirat, S. Jamil, Y. H. Mohd Yasin, J. Jalil and J. A. Jamal, Phytomedicine, 2008, 15, 306.

184 I. Jantan, M. Pisar, H. M. Sirat, N. Basar, S. Jamil, R. M. Ali and J. Jalil, Phytother. Res., 2004, 18, 1005.

185 H. Zhang, L. X. Xu, P. Wu and X. Y. Wei, Redai Yaredai Zhiwu Xuebao, 2014, 22, 89.

186 B. R. Xin, J. F. Liu, J. Kang and W. P. Chan, Mol. Cell. Toxicol., 2014, 10, 165.

187 J. Gripenberg, E. Honkanen and K. Silander, Acta Chem. Scand., 1956, 10, 393.

188 H. Itokawa, M. Morita and S. Mihashi, Phytochemistry, 1981, 20, 2503.

189 C. T. Lin, K. J. Senthil Kumar, Y. H. Tseng, Z. J. Wang, M. Y. Pan, J. H. Xiao, S. C. Chien and S. Y. Wang, J. Agric. Food Chem., 2009, 57, 6060.

190 S. Ahmad, D. A. Israf, N. H. Lajis, K. Shaari, H. Mohamed, A. A. Wahab, K. T. Ariffin, W. Y. Hoo, N. A. Aziz, A. A. Kadir, M. R. Sulaiman and M. N. Somchit, Eur. J. Pharmacol., 2006, 538, 188.

191 H. T. Le, M. G. Phan and T. S. Phan, Tap Chi Hoa Hoc, 2007, 45, 126.

192 Z. Wei, J. Yang, Y. F. Xia, W. Z. Huang, Z. T. Wang and Y. Dai, J. Biochem. Mol. Toxicol., 2012, 26, 282.

193 C. P. Shen, J. G. Luo, M. H. Yang and L. Y. Kong, Fitoterapia, 2015, 106, 153.

194 W. Q. Yang, Y. Gao, M. Li, D. R. Miao and F. Wang, J. Asian Nat. Prod. Res., 2015, 17, 783.

195 M. A. Mpalantinos, R. S. De Moura, J. P. Parente and R. M. Kuster, Phytother. Res., 1998, 12, 442.

196 Y. Y. Li, G. X. Chou and Z. T. Wang, Zhongguo Tianran Yaowu, 2009, 7, 417.

197 T. Masuda, S. Mizuguchi, T. Tanaka, K. Iritani, Y. Takeda and S. Yonemori, J. Agric. Food Chem., 2000, 48, 1479.

198 N. Nakatani, Abstracts of Papers, 223rd ACS National Meeting, AGFD, Orlando, FL, United States, April 7-11, 2002, p. 2002.

199 S. B. Jaju, N. H. Indurwade, D. M. Sakarkar, N. K. Fuloria, M. D. Ali, S. Das and S. P. Basu, Trop. J. Pharm. Res., 2009, 8, 545 .

200 B. R. Barik, A. B. Kundu and A. K. Dey, Phytochemistry, 1987, 26, 2126.

201 S. H. Shi, C. N. Zhang, A. J. Liu, H. Li, K. S. Bi and Y. Jia, Zhongguo Shiyan Fangjixue Zazhi, 2013, 19, 97.

202 J. W. Nam, S. J. Kim, A. R. Han, S. K. Lee and E. K. Seo, J. Appl. Pharmacol., 2005, 13, 263.

203 L. Zhao, L. Y. Chen and J. Y. Liang, Chin. J. Nat. Med., 2012, 10, 370 .

204 H. Matsuda, T. Morikawa, H. Managi and M. Yoshikawa, Bioorg. Med. Chem. Lett., 2003, 13, 3197.

205 S. K. Roy, S. Pahwa, H. Nandanwar and S. M. Jachak, Fitoterapia, 2012, 83, 1248.
206 T. Noro, T. Sekiya, M. Katoh, Y. Oda, T. Miyase, M. Kuroyanagi, A. Ueno and S. Fukushima, Chem. Pharm. Bull., 1988, 36, 244.

207 N. Sukhirun, W. Pluempanupat, V. Bullangpoti and O. Koul, J. Econ. Entomol., 2011, 104, 1534.

208 N. Hasima, L. I. L. Aun, M. N. Azmi, A. N. Aziz, E. Thirthagiri, H. Ibrahim and K. Awang, Phytomedicine, 2010, 17, 935.

209 H. Matsuda, Y. Pongpiriyadacha, T. Morikawa, M. Ochi and M. Yoshikawa, Eur. J. Pharmacol., 2003, 471, 59.

210 Y. Ye and B. Li, J. Gen. Virol., 2006, 87, 2047.

211 H. J. Min, J. W. Nam, E. S. Yu, J. H. Hong, E. K. Seo and E. S. Hwang, Int. Immunopharmacol., 2009, 9, 448.

212 R. Mongkol, W. Chavasiri, M. Ishida, K. Matsuda and M. Morimoto, Weed Biol. Manage., 2015, 15, 87.

213 H. C. Wang, J. X. Li, H. Li, L. W. Yang, M. H. Gao and H. A. Chen, Yaoxue Yanjiu, 2013, 32, 559.

214 W. Wang, S. Qi, H. Zhong and Q. Yao, Shipin Yu Yaopin, 2012, 14, 88.

215 S. Guan, Y.-M. Bao, B. Jiang and L.-J. An, Eur. J. Pharmacol., 2006, 538, 73.

216 S. Guan, B. Jiang, Y. M. Bao and L. J. An, Food Chem. Toxicol., 2006, 44, 1659.

217 L. J. An, S. Guan, G. F. Shi, Y. M. Bao, Y. L. Duan and B. Jiang, Food Chem. Toxicol., 2006, 44, 436.

218 G. F. Shi, L. J. An, B. Jiang, S. Guan and Y. M. Bao, Neurosci. Lett., 2006, 403, 206.

219 Y. M. Liu, B. Jiang, Y. M. Bao and L. J. An, Toxicol. in Vitro, 2008, 22, 430.

220 H. Zhao, J. Xu and S. Yang, Yunnan Nongye Daxue Xuebao, 2014, 29, 468.

221 M. G. Phan and T. S. Phan, Tap Chi Hoa Hoc, 2004, 42, 376.

222 P. T. B. Tu and S. Tawata, Molecules, 2014, 19, 16656.

223 T. Fujita, H. Nishimura, K. Kaburagi and J. Mizutani, Phytochemistry, 1994, 36, 23.

224 J. Chompoo, A. Upadhyay, M. Fukuta and S. Tawata, BMC Complementary Altern. Med., 2012, 12, 106.

225 S. B. Jaju, N. H. Indurwade, D. M. Sakarkar, N. K. Fuloria, M. D. Ali and S. P. Basu, Pharmacogn. Res., 2010, 2, 264.

226 X. Wang, X. Yang and J. Li, Zhongyaocai, 2008, 31, 853.

227 C. Qiao, X. Hao, Z. Wang and L. Xu, Zhongguo Zhongyao Zazhi, 2002, 27, 130.

228 L. Di, Z. Wang, Z. Wang, N. Li and K. Wang, Zhiwu Ziyuan Yu Huanjing Xuebao, 2011, 20, 94.

229 S. Qi, F. Ji and Q. Yao, Shipin Yu Yaopin, 2010, 12, 39.

230 N. An, J. Lin, S. Yang, Z. Zou and L. Xu, Yaoxue Xuebao, 2006, 41, 233.

231 A. Liu, X. Zhao, H. Li, Z. Liu, B. Liu, X. Mao, L. Guo, K. Bi and Y. Jia, Int. Immunopharmacol., 2014, 23, 719.

232 P. G. Ray and S. K. Majumdar, Indian J. Exp. Biol., 1976, 14, 712.

233 J. J. C. Scheffer, A. Gani and A. Baerheim Svendsen, Planta Med., 1981, 42, 140.

234 H. Haraguchi, Y. Kuwata, K. Inada, K. Shingu, K. Miyahara, M. Nagao and A. Yagi, Planta Med., 1996, 62, 308.

235 K. Chukanhom, P. Borisuthpeth and K. Hatai, Biocontrol Sci., 2005, 10, 105. 
236 M. M. Yusoff, H. Ibrahim and N. A. Hamid, Chem. Biodiversity, 2011, 8, 916.

237 S. E. Lee, H. T. Shin, H. J. Hwang and J. H. Kim, Phytother. Res., 2003, 17, 1041.

238 W. Y. Hsu, A. Simonne, A. Weissman and J. M. Kim, Food Sci. Biotechnol., 2010, 19, 873.

239 C. A. Raj, P. Ragavendran, D. Sophia, T. Starlin, M. A. Rathi and V. K. Gopalakrishnan, Chin. J. Integr. Med., 2014, 1, DOI: $10.1007 / \mathrm{s} 11655-014-1762-1$.

240 P. Muangnoi, M. Lu, J. Lee, A. Thepouyporn, R. Mirzayans, X. C. Le, M. Weinfeld and S. Changbumrung, Planta Med., 2007, 73, 748.

241 C. W. Phang, S. Malek and H. Ibrahim, BMC Complementary Altern. Med., 2013, 13, 1.

242 C. L. Hsu, Y. S. Yu and G. C. Yen, J. Agric. Food Chem., 2009, 58, 2201.

243 A. Reddy, S. Malek, H. Ibrahim and K. Sim, BMC Complementary Altern. Med., 2013, 13, 1.

244 T. P. T. Be, J. Chompoo and S. Tawata, Drug Discoveries Ther., 2015, 9, 197.
245 W. Fan, C. Z. Wang, X. L. Bao, H. H. Yuan and M. B. Lan, Asian J. Chem., 2015, 27, 532.

246 M. Y. Lee, C. S. Seo, J. A. Lee, I. S. Shin, S. J. Kim, H. K. Ha and H. K. Shin, Inflammation, 2012, 35, 746.

247 Y. S. Yu, C. L. Hsu and G. C. Yen, J. Agric. Food Chem., 2009, 57, 7673.

248 X. Yu, Y. W. Ceballos, H. Zhao and Z. Xu, Neurosci. Res. Commun., 2003, 33, 105.

249 J. C. Hanish Singh, V. Alagarsamy, P. V. Diwan, S. Sathesh Kumar, J. C. Nisha and Y. Narsimha Reddy, J. Ethnopharmacol., 2011, 138, 85.

250 Z. J. Zhang, L. C. V. Cheang, M. W. Wang, G. H. Li, I. Chu, Z. X. Lin and S. M. Y. Lee, Cell. Mol. Neurobiol., 2012, 32, 27.

251 F. M. Hammouda, S. S. El-Hawary, H. A. Kassem, W. A. Tawfik, A. A. Abdel Motaal, N. M. Nazif and S. S. ElShamy, Res. J. Pharm., Biol. Chem. Sci., 2015, 6, 448.

252 C. R. Achuthan and J. Padikkala, Indian J. Clin. Biochem., 1997, 12, 55. 